\title{
Sharp estimates for the arithmetic Nullstellensatz
}

\author{
Teresa Krick円, Luis Miguel Pardođ, and Martín Sombra ${ }^{1}$
}

\begin{abstract}
We present sharp estimates for the degree and the height of the polynomials in the Nullstellensatz over $\mathbb{Z}$. The result improves previous work of Philippon, Berenstein-Yger and Krick-Pardo.

We also present degree and height estimates of intrinsic type, which depend mainly on the degree and the height of the input polynomial system. As an application, we derive an effective arithmetic Nullstellensatz for sparse polynomial systems.

The proof of these results relies heavily on the notion of local height of an affine variety defined over a number field. We introduce this notion and study its basic properties.
\end{abstract}

Keywords. Height of varieties, Chow forms, arithmetic Nullstellensatz, intrinsic parameters, sparse elimination theory.

AMS Subject Classification. Primary: 11G35, Secondary: 13P10.

\section{Contents}

$\begin{array}{lr}\text { Introduction } & 2\end{array}$

1 Height of polynomials and varieties

1.1 Height of polynomials . . . . . . . . . . . . . . . . . . . . . . . . 6

1.1.1 Height of polynomials over $\mathbb{C}_{v}$. . . . . . . . . . . . . . . . . . . . . 7

1.1 .2 Height of polynomials over a number field _ . . . . . . . . . . . . . . . . . . 9

1.2 Height of varieties $\ldots \ldots \ldots \ldots \ldots \ldots \ldots \ldots$

1.2 .1 Degree of varieties . . . . . . . . . . . . . . . . . . . . . . . . . . . . . . . . . . . . . . . . .

1.2 .2 Normalization of Chow forms . . . . . . . . . . . . . . . . . . . . . . . 12

1.2 .3 Height of varieties over $\mathbb{C}_{v}$. . . . . . . . . . . . . . . . . . . . . . . 13

1.2 .4 Height of varieties over a number field . . . . . . . . . . . . . . . . . . . . . . 14

1.2 .5 Height of affine toric varieties . . . . . . . . . . . . . . . . . . . . . . 14

2 Estimates for local and global heights 16

2.1 Estimates for Chow forms $\ldots \ldots \ldots \ldots$. . . . . . . . . . . . . . 16

2.1.1 Generalized Chow forms . . . . . . . . . . . . . . . . . . . . 16

2.1 .2 An estimate for generalized Chow forms . . . . . . . . . . . . . . . . . . . . . 17

2.2 Basic properties of the height . . . . . . . . . . . . . . . . . . . . . . . . . 19

2.2.1 Height of varieties under affine maps _. . . . . . . . . . . . . . . . . . . . . 19

$2.2 .2 \quad$ Local height of the intersection of varieties . . . . . . . . . . . . . . . . . . . 22

2.2 .3 An arithmetic Bernstein-Kushnirenko theorem . . . . . . . . . . . . . . . . . 24

2.3 Local height of norms and traces . . . . . . . . . . . . . . . . . . . 25

2.3 .1 Characteristic polynomials . . . . . . . . . . . . . . . . . 25

2.3 .2 Estimates for norms and traces . . . . . . . . . . . . . . . . . . . . 27

$3 \quad$ An effective arithmetic Nullstellensatz 29

3.1 Division modulo complete intersection ideals . . . . . . . . . . . . . . . . . . . . . . 29

3.1.1 Tate trace formula . . . . . . . . . . . . . . . . . . . . . . . 29

3.1.2 A division lemma . . . . . . . . . . . . . . . . . . . . . . . 30

\footnotetext{
${ }^{1}$ T. Krick and M. Sombra were partially supported by CONICET, UBACyT and ANPCyT (Argentina), and by the Mathematical Sciences Research Institute at Berkeley (USA).

M. Sombra was also partially supported by grant NSF-DMS 97-29992 to the Institute for Advanced Study at Princeton (USA).

${ }^{2}$ L. M. Pardo was partially supported by PB 96-0671-C02-02 (Spain), and by CNRS 1026 MEDICIS (France).
} 
$3.2 \quad$ An effective arithmetic Nullstellensatt . . . . . . . . . . . . . . . . . . . . . . 34

3.2.1 Estimates for the complete intersection case . . . . . . . . . . . . . . . 34

3.2 .2 Proof of Theorem $1 \ldots \ldots \ldots \ldots \ldots$

\begin{tabular}{|lll}
\hline & Intrinsic type estimates & 41
\end{tabular}

4.1 Equations in general position . . . . . . . . . . . . . . . . . . . 42

$4.1 .1 \quad$ An effective Bertini theorem . . . . . . . . . . . . . . . . . . 42

4.1 .2 Effective Noether normal position . . . . . . . . . . . . . . . . 46

4.2 An intrinsic arithmetic Nullstellensatz . . . . . . . . . . . . . . . . . . 47

4.2 .1 Intrinsic parameters . . . . . . . . . . . . . . . . . 47

$4.2 .2 \quad$ Proof of Theorem 2 . . . . . . . . . . . . . . . . . . . . . . . . . . 49

$4.2 .3 \quad$ Estimates for the sparse case $\ldots \ldots \ldots \ldots \ldots \ldots$. . . . . . . . . . . . 51

\begin{tabular}{ll}
\hline References & 52
\end{tabular}

\section{Introduction}

Hilbert Nullstellensatz is a cornerstone of algebraic geometry. Under a simplified form, its statement is the following:

Let $f_{1}, \ldots, f_{s} \in \mathbb{Z}\left[x_{1}, \ldots, x_{n}\right]$ be polynomials such that the equation system

$$
f_{1}(x)=0, \ldots, f_{s}(x)=0
$$

has no solution in $\mathbb{C}^{n}$. Then there exist $a \in \mathbb{Z} \backslash\{0\}$ and $g_{1}, \ldots, g_{s} \in \mathbb{Z}\left[x_{1}, \ldots, x_{n}\right]$ satisfying the Bézout identity

$$
a=g_{1} f_{1}+\cdots+g_{s} f_{s}
$$

As for many central results in commutative algebra and algebraic geometry, it is an existential non-effective statement. The estimation of both the degree and the height of polynomials satisfying Identity (2) became an important and widely considered question. Effective versions of Hilbert Nullstellensatz apply to a wide range of situations in number theory and theoretical computer science. In particular, they decide the consistency of a given polynomial system. In their arithmetic presentation, they apply to Lojasiewicz inequalities [48], [23] and to the consistency problem over finite fields [25], 19].

We recall that the height $h(f)$ of a polynomial $f \in \mathbb{Z}\left[x_{1}, \ldots, x_{n}\right]$ is defined as the logarithm of the maximum modulus of its coefficients. The main result of this paper is the following effective arithmetic Nullstellensatz:

Theorem 1 Let $f_{1}, \ldots, f_{s} \in \mathbb{Z}\left[x_{1}, \ldots, x_{n}\right]$ be polynomials without common zeros in $\mathbb{C}^{n}$. Set $d:=\max _{i} \operatorname{deg} f_{i}$ and $h:=\max _{i} h\left(f_{i}\right)$.

Then there exist $a \in \mathbb{Z} \backslash\{0\}$ and $g_{1}, \ldots, g_{s} \in \mathbb{Z}\left[x_{1}, \ldots, x_{n}\right]$ such that

- $a=g_{1} f_{1}+\cdots+g_{s} f_{s}$,

- $\operatorname{deg} g_{i} \leq 4 n d^{n}$,

- $h(a), h\left(g_{i}\right) \leq 4 n(n+1) d^{n}(h+\log s+(n+7) \log (n+1) d)$. 
As we will see below, this result substantially improves all previously known estimates for the arithmetic Nullstellensatz.

The following variant of a well-known example due to Masser and Philippon [6] yields a lower bound for any general degree and height estimate. Set

$$
f_{1}:=x_{1}^{d}, f_{2}:=x_{1} x_{n}^{d-1}-x_{2}^{d}, \ldots, f_{n-1}:=x_{n-2} x_{n}^{d-1}-x_{n-1}^{d}, f_{n}:=x_{n-1} x_{n}^{d-1}-H
$$

for any $n, d, H \in \mathbb{N}$. These are polynomials of degree $d$ and height bounded by $h:=\log H$ without common zeros in $\mathbb{C}^{n}$. Let $a \in \mathbb{Z} \backslash\{0\}$ and $g_{1}, \ldots, g_{n} \in \mathbb{Z}\left[x_{1}, \ldots, x_{n}\right]$ such that

$$
a=g_{1} f_{1}+\cdots+g_{n} f_{n} .
$$

Specializing this identity at $x_{1}:=H^{d^{n-2}} t^{d^{n-1}-1}, \ldots, x_{n-1}:=H t^{d-1}, x_{n}:=1 / t$ we obtain

$$
a=g_{1}\left(H^{d^{n-2}} t^{d^{n-1}-1}, \ldots, H t^{d-1}, 1 / t\right) H^{d^{n-1}} t^{d^{n}-d} .
$$

We conclude that $\operatorname{deg} g_{1} \geq d^{n}-d$ and $h(a) \geq d^{n-1} h$. In fact, a modified version of this example gives the improved lower bound $h(a) \geq d^{n} h$ (Example 3.10). This shows that our estimate is essentially optimal.

The earlier work on the effective Nullstellensatz dealt with the degree bounds. Let $k$ be a field and let $f_{1}, \ldots, f_{s} \in k\left[x_{1}, \ldots, x_{n}\right]$ be polynomials of degree bounded by $d$ without common zeros in $\bar{k}^{n}$.

In 1926, Hermann [22] (see also [20], 40]) proved that there exist $g_{1}, \ldots, g_{s} \in k\left[x_{1}, \ldots, x_{n}\right]$ such that

$$
1=g_{1} f_{1}+\cdots+g_{s} f_{s}
$$

with $\operatorname{deg} g_{i} f_{i} \leq 2(2 d)^{2^{n-1}}$.

After a conjecture of Keller and Gröbner, this estimate was dramatically improved by Brownawell [6] to $\operatorname{deg} g_{i} f_{i} \leq n^{2} d^{n}+n d$ in case $\operatorname{char}(k)=0$, while Caniglia, Galligo and Heintz [7] showed that $\operatorname{deg} g_{i} f_{i} \leq d^{n^{2}}$ holds in the general case.

These results were then independently refined by Kollár [26] and by Fitchas and Galligo [1] to

$$
\operatorname{deg} g_{i} f_{i} \leq \max \{3, d\}^{n},
$$

which is optimal in case $d \geq 3$. For $d=2$, Sombra [50 recently showed that the bound $\operatorname{deg} g_{i} f_{i} \leq$ $2^{n+1}$ holds.

Now, let us consider the height aspect: assume $f_{1}, \ldots, f_{s} \in \mathbb{Z}\left[x_{1}, \ldots, x_{n}\right]$ are polynomials of degree and height bounded by $d$ and $h$, respectively. The previous degree bound reduces Bézout identity (2) to a system of $\mathbb{Q}$ - linear equations, which can be solved by Cramer rule. The obtained estimate for the height of the integer $a$ and the polynomials $g_{i}$ is of type $s d^{n^{2}}(h+\log s+d)$.

However, it was soon conjectured that the true height bound should be much smaller.

Philippon [45] obtained the following sharper estimate for the denominator $a$ in the Bézout equation:

$$
\operatorname{deg} g_{i} \leq(n+2) d^{n} \quad, \quad h(a) \leq \kappa(n) d^{n}(h+d),
$$

where $\kappa(n)$ depends exponentially on $n$.

The first essential progress on height estimates for all the polynomials $g_{i}$ was achieved by Berenstein and Yger [2], who obtained

$$
\operatorname{deg} g_{i} \leq n(2 n+1) d^{n} \quad, \quad h(a), h\left(g_{i}\right) \leq \lambda(n) d^{8 n+3}(h+\log s+d \log d),
$$

where $\lambda(n)$ is a (non-explicit) constant which depends exponentially on $n$. Their proof relies on the previous work of Philippon and on techniques from complex analysis. 
Later on, Krick and Pardo [28], 29] obtained

$$
\operatorname{deg} g_{i} \leq(n d)^{c n} \quad, \quad h(a), h\left(g_{i}\right) \leq(n d)^{c n}(h+\log s+d),
$$

where $c$ is a universal constant $(c \leq 35)$. Their proof, based on duality theory for Gorenstein algebras, is completely algebraic.

Finally, Berenstein and Yger [3] improved their height bound to $\lambda(n) d^{4 n+2}(h+\log s+d)$, and extended it to the case when $\mathbb{Z}$ is replaced by an arbitrary diophantine ring. It should be said, however, that the possibility of such an extension was already clear from the arguments of [29].

We refer the reader to the surveys [55], [1], [42] for a broad introduction to the history of the effective Nullstellensatz, main results and open questions. Aside from degree and height estimates, there is a strong current area of research on computational issues [17, [12], [29], [16], [15], [19]. There are other results in the recent research papers [47], [27], [9].

With respect to previous work, in this paper we improve in an almost optimal way the dependence of the height estimate on $d^{n}$ and we eliminate the extraneous exponential constants depending on $n$. We remark that the polynomials arising in Theorem 1 are a slight variant of the polynomials which appear in [29] and can thus be effectively computed by their algorithm.

Although the exponential behavior of the degree and height estimates is - in the worst-case unavoidable, it has been observed that there are many particular instances in which these estimates can be essentially improved. This has motivated the introduction of parameters associated to the input system which identify special families whose behavior with respect to our problem is polynomial instead of exponential.

In this spirit, Giusti et al. 16 introduced the notion of degree of a polynomial system $f_{1}, \ldots, f_{s}$. Roughly speaking, this parameter measures the degree of the varieties cut out by $f_{1}, \ldots, f_{i}$ for $i=1, \ldots, s-1$. It was soon realized that the degrees in the Nullstellensatz can be controlled in terms of this parameter, giving rise to the so-called "intrinsic Nullstellensätze" [16], [30, [15], 49]. Recently Hägele, Morais, Pardo and Sombra [19] (see also [18]) obtained an arithmetic analogue of these intrinsic Nullstellensätze. To this aim, they introduced the notion of height of a polynomial system, the arithmetic analogue of the degree of the system. They obtained degree and height estimates which depend polynomially on the number of variables and on the degree, height and complexity of the input system. This result followed from their study of the computational complexity of the Nullstellensatz.

In this paper we obtain a dramatical improvement over this result, bringing it to an (apparently) almost optimal form. In particular, we show that the dependence on the degree and the height of the system is linear, and we eliminate the influence of the complexity of the input.

Theorem 2 Let $f_{1}, \ldots, f_{s} \in \mathbb{Z}\left[x_{1}, \ldots, x_{n}\right]$ be polynomials without common zeros in $\mathbb{C}^{n}$. Set $d:=\max _{i} \operatorname{deg} f_{i}$ and $h:=\max _{i} h\left(f_{i}\right)$. Let $\delta$ and $\eta$ denote the degree and the height of the polynomial system $f_{1}, \ldots, f_{s}$.

Then there exist $a \in \mathbb{Z} \backslash\{0\}$ and $g_{1}, \ldots, g_{s} \in \mathbb{Z}\left[x_{1}, \ldots, x_{n}\right]$ such that

- $a=g_{1} f_{1}+\cdots+g_{s} f_{s}$,

- $\operatorname{deg} g_{i} \leq 2 n^{2} d \delta$,

- $h(a), h\left(g_{i}\right) \leq(n+1)^{2} d\left(2 \eta+(h+\log s) \delta+21(n+1)^{2} d \log (d+1) \delta\right)$.

Since $\delta \leq d^{n-1}$ and $\eta \leq n d^{n-1}(h+\log s+3 n(n+1) d)$ (Lemma 4.8) one recovers from this statement essentially the same estimates of Theorem 1 . However, we remark that Theorem 2 is a more flexible result, as there are many situations in which the degree and the height of the input 
system are smaller than the Bézout bounds. When this is the case, it yields a much more accurate estimate (Subsection 4.2.2).

As an application of Theorem 2 we derive an arithmetic effective Nullstellensatz for sparse polynomial systems. To state this result, we first need to introduce some standard notation.

The support $\operatorname{Supp}\left(f_{1}, \ldots, f_{s}\right)$ of a polynomial system $f_{1}, \ldots, f_{s} \subset \mathbb{C}\left[x_{1}, \ldots, x_{n}\right]$ is defined as the set of exponents of all the non-zero monomials of all $f_{i}$ 's, and the Newton polytope $\mathcal{N}\left(f_{1}, \ldots, f_{s}\right)$ is the convex hull of this support. The (normalized) volume of $f_{1}, \ldots, f_{s}$ equals $n$ ! times the volume of the corresponding Newton polytope.

The notions of Newton polytope and volume of a polynomial system give a sharper characterization of its monomial structure than the degree alone. These concepts were introduced in the context of root counting by Bernstein [4] and Kushnirenko [32], and are now in the basis of sparse elimination theory (see e.g. [53]).

We obtain the following result:

Corollary 3 Let $f_{1}, \ldots, f_{s} \in \mathbb{Z}\left[x_{1}, \ldots, x_{n}\right]$ be polynomials without common zeros in $\mathbb{C}^{n}$. Set $d:=\max _{i} \operatorname{deg} f_{i}$ and $h:=\max _{i} h\left(f_{i}\right)$. Let $\mathcal{V}$ denote the volume of the polynomial system $1, x_{1}, \ldots, x_{n}, f_{1}, \ldots, f_{s}$.

Then there exist $a \in \mathbb{Z} \backslash\{0\}$ and $g_{1}, \ldots, g_{s} \in \mathbb{Z}\left[x_{1}, \ldots, x_{n}\right]$ such that

- $a=g_{1} f_{1}+\cdots+g_{s} f_{s}$,

- $\operatorname{deg} g_{i} \leq 2 n^{2} d \mathcal{V}$,

- $h(a), h\left(g_{i}\right) \leq 2(n+1)^{3} d \mathcal{V}\left(h+\log s+2^{2 n+4} d \log (d+1)\right)$.

The crucial observation here is that both the degree and the height of a polynomial system are essentially controlled by the normalized volume. This follows from an adequate arithmetic version of the Bernstein-Kushnirenko theorem (Proposition 2.11). Our result follows then from Theorem 2 in a straightforward way.

As before, we can apply the worst-case bound $\mathcal{V} \leq d^{n}$ to recover from this result an estimate similar to the one presented in Theorem 1. However, this result gives sharper estimates for both the degree and the height when the input system is sparse (Example 4.13).

The sparse aspect in the Nullstellensatz was previously considered by Canny and Emiris [8] for the case of $n+1 n$-variate Laurent polynomials without common roots at toric infinity. Their result is the sparse analogue of Macaulay effective Nullstellensatz (see e.g. 34]). The first general sparse Nullstellensatz was obtained by Sombra [50]. In both cases the authors give bounds for the Newton polytopes of the output polynomials in terms of the Newton polytopes of the input ones. We refer to the original papers for the exact statements.

It is quite difficult to make a definite comparison between these results and ours. The latter does not give sharp bounds for Newton polytopes. But on the other hand, our degree estimate for the general case is better, while the height estimate is completely new.

The key ingredient in our treatment of the arithmetic Nullstellensatz is the notion of local height of a variety defined over a number field $K$.

Let $V \subset \mathbb{A}^{n}(\overline{\mathbb{Q}})$ be an equidimensional affine variety defined over $K$. For each absolute value $v$ over $K$, we introduce the local height $h_{v}(V)$ of $V$ at $v$ as a Mahler measure of a suitable normalized Chow form of $V$. This definition is consistent with the Faltings height $h(V)$ of $V$, namely:

$$
h(V)=\frac{1}{[K: \mathbb{Q}]} \sum_{v \in M_{K}} N_{v} h_{v}(V)
$$


where $M_{K}$ denotes the set of canonical absolute values of $K$, and $N_{v}$ the multiplicity of $v$.

We study the basic properties of this notion. In particular we are able to estimate the local height of the trace and the norm of a polynomial $f \in K\left[x_{1}, \ldots, x_{n}\right]$ with respect to an integral extension $K\left[\mathrm{~A}^{r}\right] \hookrightarrow K[V]$. We also obtain local analogues of many of the global results of Bost, Gillet and Soulé [5] and Philippon [46].

Our proof of the arithmetic Nullstellensatz is based on duality theory for Gorenstein algebras (Tate trace formula). This technique was introduced in the context of the effective Nullstellensatz in [17], [12]. Here, we follow mostly the lines of Sabia-Solernó [47] and Krick-Pardo [29.

The trace formula allows to perform division modulo complete intersection ideals, with good control of the degree and height of the involved polynomials. The local arithmetic intersection theory plays, with respect to the height estimates, the role of the classical intersection theory with respect to the degree bounds.

Finally, we remark that all of our results are valid not just for $\mathbb{Q}$ but for arbitrary number fields. Moreover, it is quite evident from our arguments that they can be extended to any product formula field.

In fact, the general analysis over number fields is necessary to obtain the sharpest estimates for the case $K:=\mathbb{Q}$. We also remark that the estimates in the general version of Theorem 1 do not depend on the involved number field.

The outline of the paper is the following:

In Chapter 1, we recall the basic definitions and properties of the height of polynomials, and we introduce the notion of local height of a variety defined over a number field.

In Chapter 2, we derive useful estimates for the local heights of the trace and the norm of a polynomial in $K[V]$, and we study the behavior of the local heights of the intersection of a variety with a hypersurface.

In Chapter 3, we recall the basic facts of duality theory which will be useful in our context, and we prove Theorem 1.

In Chapter 4, we focus on the intrinsic and sparse versions of the arithmetic Nullstellensatz.

\section{Height of polynomials and varieties}

Throughout this paper $\mathbb{Q}$ denotes the field of rational numbers, $\mathbb{Z}$ the ring of rational integers, $K$ a number field, and $\mathcal{O}_{K}$ its ring of integers. We also denote by $\mathbb{R}$ the field of real numbers, $\mathbb{C}$ the field of complex numbers, $k$ an arbitrary field, and $\bar{k}$ an algebraic closure of $k$. As usual, $I^{n}$ and $\mathbb{P}^{n}$ will denote the affine and the projective space of $n$ dimensions over $\bar{k}$, respectively. For every rational prime $p$ we denote by $|\cdot|_{p}$ the corresponding $p$-adic absolute value over $\mathbb{Q}$. We also denote the ordinary absolute value by $|\cdot|_{\infty}$ or simply by $|\cdot|$. These form a complete set of independent absolute values over $\mathbb{Q}$ : we identify the set $M_{Q}$ of these absolute values with the set $\{\infty, p ; p$ prime $\}$.

For $v \in M_{Q}$ we denote by $\mathbb{Q}_{v}$ the completion of $\mathbb{Q}$ with respect to the absolute value $v$. In case $v=\infty$ we have $\mathbb{Q}_{\infty}=\mathbb{R}$, while in case $p$ is prime, we have that $\mathbb{Q}_{p}$ is the $p$-adic field. There exists a unique extension of $v$ to an absolute value over the algebraic closure $\mathbb{C}_{v}$ of $\mathbb{Q}_{v}$, which we denote by $v$. In case $v=\infty$ we have $\mathbb{C}_{\infty}=\mathbb{C}$.

\subsection{Height of polynomials}

In this section we introduce the different measures for the size of a multivariate polynomial, both over $\mathbb{C}_{v}$ and over a number field. We establish the link between the different notions and study their basic properties. 


\subsubsection{Height of polynomials over $\mathbb{C}_{v}$}

We fix an absolute value $v \in\{\infty, p ; p$ prime $\}$ for the rest of this chapter. Let $\mathcal{A} \subset \mathbb{C}_{v}$ be a finite set. The absolute value of $\mathcal{A}$ is defined as

$$
|\mathcal{A}|_{v}:=\max \left\{|a|_{v}, a \in \mathcal{A}\right\}
$$

and the (logarithmic) height of $\mathcal{A}$ as

$$
h_{v}(\mathcal{A}):=\max \left\{0, \log |\mathcal{A}|_{v}\right\} .
$$

For a polynomial $f=\sum_{\alpha} a_{\alpha} x^{\alpha} \in \mathbb{C}_{v}\left[x_{1}, \ldots, x_{n}\right]$, we define the absolute value of $f$ (denoted by $\left.|f|_{v}\right)$ as the absolute value of its set of coefficients, and the height of $f$ (denoted by $h_{v}(f)$ ) as the height of its set of coefficients. That is

$$
\begin{aligned}
|f|_{v} & :=\max _{\alpha}\left\{\left|a_{\alpha}\right|_{v}\right\}, \\
h_{v}(f) & :=\max \left\{0, \log |f|_{v}\right\} .
\end{aligned}
$$

When $v=\infty$, i.e. when $f$ has complex coefficients, we shall make use of the (logarithmic) Mahler measure of $f$ defined as

$$
m(f):=\int_{0}^{1} \ldots \int_{0}^{1} \log \left|f\left(e^{2 \pi i t_{1}}, \ldots, e^{2 \pi i t_{n}}\right)\right| d t_{1} \ldots d t_{n} .
$$

This integral is well-defined, as $\log |f|$ is a plurisubharmonic function on $\mathbb{C}^{n}$ [37, Appendix I]. The Mahler measure was introduced by Lehmer [35] for the case of a univariate polynomial $f:=$ $a_{d} \prod_{i=1}^{d}\left(x-\alpha_{i}\right) \in \mathbb{C}[x]$ as

$$
m(f)=\log \left|a_{d}\right|+\sum_{i=1}^{d} \max \left\{0, \log \left|\alpha_{i}\right|\right\} .
$$

The link between both expressions of $m(f)$ is given by Jensen formula. The general case was introduced and studied by Mahler [38].

The key property of the Mahler measure is its additivity:

$$
m(f g)=m(f)+m(g) .
$$

We have the following relation between $\log |f|$ and $m(f)$ :

$$
-\log (n+1) \operatorname{deg} f \leq m(f)-\log |f| \leq \log (n+1) \operatorname{deg} f .
$$

The right inequality follows from the definition of $m$ and the fact that the number of monomials of $f$ is bounded by $\left(\begin{array}{c}n+\operatorname{deg} f \\ n\end{array}\right) \leq(n+1)^{\operatorname{deg} f}$. For the left inequality, we refer to 44, Lemme 1.13] and its proof.

When $f$ has total degree bounded by 1 , the inequality is refined to $\log |f| \leq m(f)$. Also, for any degree, $m\left(f\left(x_{1}, \ldots, x_{n-1}, 0\right)\right) \leq m(f)$.

We shall make frequent use of the following more precise relation:

Lemma 1.1 Let $f \in \mathbb{C}\left[X_{1}, \ldots, X_{r}\right]$ be a polynomial in $r$ groups of $n_{i}$ variables each. Let $d_{i}$ denote the degree of $f$ in the group of variables $X_{i}$. Then

$$
-\sum_{i=1}^{r} \log \left(n_{i}+1\right) d_{i} \leq m(f)-\log |f| \leq \sum_{i=1}^{r} \log \left(n_{i}+1\right) d_{i} .
$$


Proof.- The right inequality follows directly from the definition of $m(f)$ and the fact that we can bound by $\prod_{i}\left(n_{i}+1\right)^{d_{i}}$ the number of monomials of $f$. Thus we only consider the left inequality. Let $f_{\alpha_{1} \cdots \alpha_{i}} \in \mathbb{C}\left[X_{i+1}, \ldots, X_{r}\right]$ denote the coefficient of $f$ with respect to the monomial $X_{1}^{\alpha_{1}} \cdots X_{i}^{\alpha_{i}}$. Applying Inequality (1.1) we obtain for all $\left(\xi_{i+1}, \ldots, \xi_{r}\right) \in \mathbb{C}^{n_{i+1}+\cdots+n_{r}}$ :

$$
\log \left|f_{\alpha_{1} \cdots \alpha_{i-1}}\left(X_{i}, \xi_{i+1}, \ldots, \xi_{r}\right)\right| \leq m\left(f_{\alpha_{1} \cdots \alpha_{i-1}}\left(X_{i}, \xi_{i+1}, \ldots, \xi_{r}\right)\right)+\log \left(n_{i}+1\right) d_{i} .
$$

We have $\left|f_{\alpha_{1} \cdots \alpha_{i-1}}\left(X_{i}, \xi_{i+1}, \ldots, \xi_{r}\right)\right|=\max _{\alpha_{i}}\left|f_{\alpha_{1} \cdots \alpha_{i}}\left(\xi_{i+1}, \ldots, \xi_{r}\right)\right|$. We integrate both sides of the last inequality on $S_{1}^{n_{i+1}+\cdots+n_{r}}$ and we deduce

$$
\max _{\alpha_{i}} m\left(f_{\alpha_{1} \cdots \alpha_{i}}\right) \leq m\left(f_{\alpha_{1} \cdots \alpha_{i-1}}\right)+\log \left(n_{i}+1\right) d_{i}
$$

The statement follows then by induction and the fact that $\log |f|_{v}=\max \left\{m\left(f_{\alpha_{1} \cdots \alpha_{r}}\right) ; \alpha_{i} \in \mathbb{Z}^{n_{i}}\right\}$.

Let $f \in \mathbb{C}\left[X_{1}, \ldots, X_{r}\right]$ be a multihomogeneous polynomial in $r$ groups of $n_{i}+1$ each, and set $f^{a}$ for a deshomogenization of $f$ with respect to these groups of variables. Then $m\left(f^{a}\right)=m(f)$, $\log \left|f^{a}\right|=\log |f|$. Thus the estimates of the preceding lemma also hold for $f$.

Next we introduce the (logarithmic) $S_{n}$-Mahler measure of a polynomial $f \in \mathbb{C}\left[x_{1}, \ldots, x_{n}\right]$ as

$$
m\left(f ; S_{n}\right):=\int_{S_{n}} \log |f| \mu_{n},
$$

where $S_{n}:=\left\{\left(z_{1}, \ldots, z_{n}\right) \in \mathbb{C}^{n}:\left|z_{1}\right|^{2}+\cdots+\left|z_{n}\right|^{2}=1\right\}$ is the unit sphere in $\mathbb{C}^{n}$, and $\mu_{n}$ is the measure of total mass 1 , invariant with respect to the unitary group $U(n)$.

More generally, let $f \in \mathbb{C}\left[X_{1}, \ldots, X_{r}\right]$ be a polynomial in $r$ groups of $n$ variables each. Its $S_{n}^{r}$-Mahler measure is then defined as

$$
m\left(f ; S_{n}^{r}\right):=\int_{S_{n}^{r}} \log |f| \mu_{n}^{r},
$$

with $S_{n}^{r}:=S_{n} \times \cdots \times S_{n}$. This alternative Mahler measure was introduced by Philippon [46, I]. With this notation, the ordinary Mahler measure $m(f)$ of $f \in \mathbb{C}\left[x_{1}, \ldots, x_{n}\right]$ coincides with $m\left(f ; S_{1}^{n}\right)$.

The $S_{n}^{r}$-Mahler measure is related to the ordinary Mahler measure by the following inequalities [36]:

$$
0 \leq m(f)-m\left(f ; S_{n}^{r}\right) \leq r d \sum_{i=1}^{n-1} \frac{1}{2 i},
$$

where $d$ is a bound for the degree of $f$ in each group of variables.

Finally, we summarize in the following lemma the basic properties of the notion of height of polynomials in $\mathbb{C}_{v}\left[x_{1}, \ldots, x_{n}\right]$.

Lemma 1.2 Let $v \in M_{Q}$ and $f_{1}, \ldots, f_{s} \in \mathbb{C}_{v}\left[x_{1}, \ldots, x_{n}\right]$.

1. If $v=\infty$ then

(a) $h_{\infty}\left(\sum_{i} f_{i}\right) \leq \max _{i}\left\{h_{\infty}\left(f_{i}\right)\right\}+\log s$.

(b) $h_{\infty}\left(\prod_{i=1}^{s} f_{i}\right) \leq \sum_{i=1}^{s} h_{\infty}\left(f_{i}\right)+\log (n+1) \sum_{i=1}^{s-1} \operatorname{deg} f_{i}$. $h_{\infty}\left(f_{1} f_{2}\right) \leq h_{\infty}\left(f_{1}\right)+h_{\infty}\left(f_{2}\right)+\log (n+1) \min \left\{\operatorname{deg} f_{1}, \operatorname{deg} f_{2}\right\}$

(c) Let $g \in \mathbb{C}\left[y_{1}, \ldots, y_{s}\right]$. Set $d:=\max _{i}\left\{\operatorname{deg} f_{i}\right\}$ and $h_{\infty}:=\max _{i}\left\{h_{\infty}\left(f_{i}\right)\right\}$. Then

$$
h_{\infty}\left(g\left(f_{1}, \ldots, f_{s}\right)\right) \leq h_{\infty}(g)+\operatorname{deg} g\left(h_{\infty}+\log (s+1)+\log (n+1) d\right) .
$$


(d) $\log \left|\prod_{i} f_{i}\right|_{\infty} \geq \sum_{i} \log \left|f_{i}\right|_{\infty}-2 \log (n+1) \sum_{i} \operatorname{deg} f_{i}$.

2. If $v=p$ for some prime $p$ then

(a) $h_{p}\left(\sum_{i} f_{i}\right) \leq \max _{i}\left\{h_{p}\left(f_{i}\right)\right\}$.

(b) $h_{p}\left(\prod_{i} f_{i}\right) \leq \sum_{i} h_{p}\left(f_{i}\right)$.

(c) Let $g \in \mathbb{C}_{p}\left[y_{1}, \ldots, y_{s}\right]$. Set $d:=\max _{i}\left\{\operatorname{deg} f_{i}\right\}$ and $h_{p}:=\max _{i}\left\{h_{p}\left(f_{i}\right)\right\}$. Then $h_{p}\left(g\left(f_{1}, \ldots, f_{s}\right)\right) \leq h_{p}(g)+\operatorname{deg} g h_{p}$.

(d) $\log \left|\prod_{i} f_{i}\right|_{p}=\sum_{i} \log \left|f_{i}\right|_{p}$.

Proof of Lemma 1.2.- (1.a), (1.b), (2.a) and (2.b) are immediate from the definition of $h_{v}$.

(1.c) and (2.c):

Let us consider the case $v=\infty$. Set $c(n):=\log (n+1)$.

First we compute $h_{v}\left(f_{1}^{\alpha_{1}} \cdots f_{s}^{\alpha_{s}}\right)$ for the exponent $\left(\alpha_{1}, \ldots, \alpha_{s}\right)$ of a monomial of $g$. Applying (1.b) we obtain

$$
h_{\infty}\left(f_{1}^{\alpha_{1}} \cdots f_{s}^{\alpha_{s}}\right) \leq\left(c(n) d+h_{\infty}\right) \sum_{i} \alpha_{i} \leq\left(c(n) d+h_{\infty}\right) \operatorname{deg} g .
$$

The polynomial $g$ has at most $(s+1)^{\operatorname{deg} g}$ monomials and so

$$
h_{\infty}\left(g\left(f_{1}, \ldots, f_{s}\right)\right) \leq h_{\infty}(g)+\left(c(n) d+h_{\infty}\right) \operatorname{deg} g+c(s) \operatorname{deg} g .
$$

The case $v \neq \infty$ follows in a similar way.

(1.d) and (2.d):

In case $v=\infty$, we apply directly Inequality (1.1):

$$
\begin{aligned}
\sum_{i} \log \left|f_{i}\right|_{\infty} & \leq \sum_{i}\left(m\left(f_{i}\right)+c(n) \operatorname{deg} f_{i}\right) \\
& =m\left(\prod_{i} f_{i}\right)+c(n) \sum_{i} \operatorname{deg} f_{i} \\
& \leq \log \left|\prod_{i} f_{i}\right|_{\infty}+2 c(n) \sum_{i} \operatorname{deg} f_{i} .
\end{aligned}
$$

In case $v=p$, Gauss Lemma implies that $\sum_{i} \log \left|f_{i}\right|_{p}=\log \left|\prod_{i} f_{i}\right|_{p}$.

We shall make frequent use of the following particular case of the previous lemma:

Let $\left(f_{i j}\right)_{i j}$ be a $s \times s$-matrix of polynomials in $\mathbb{C}_{v}\left[x_{1}, \ldots, x_{n}\right]$ of degrees and heights bounded by $d$ and $h_{v}$ respectively. From Lemma $1.2(\mathrm{a}, \mathrm{b})$ we obtain:

- $h_{\infty}\left(\operatorname{det}\left(f_{i j}\right)_{i j}\right) \leq s\left(h_{\infty}+\log s+d \log (n+1)\right)$,

- $h_{p}\left(\operatorname{det}\left(f_{i j}\right)_{i j}\right) \leq s h_{p}$.

\subsubsection{Height of polynomials over a number field}

The set $M_{K}$ of absolute values over $K$ which extend the absolute values in $M_{Q}$ is called the canonical set. We denote by $M_{K}^{\infty}$ the set of archimedean absolute values in $M_{K}$.

If $v \in M_{K}$ extends an absolute value $v_{0} \in M_{Q}$ (which is denoted by $v \mid v_{0}$ ) there exists a (non necessarily unique) immersion $\sigma_{v}: K \hookrightarrow \mathbb{C}_{v_{0}}$ corresponding to $v$, i.e. such that $|\xi|_{v}=\left|\sigma_{v}(\xi)\right|_{v_{0}}$ for every $\xi \in K$. 
In the $p$-adic case, there is a one-to-one correspondence $\mathcal{P} \mapsto v(\mathcal{P})$ between the prime ideals of $\mathcal{O}_{K}$ which divide $p$, and absolute values extending $p$, defined by

$$
|\xi|_{v(\mathcal{P})}:=p^{-\operatorname{ord}_{\mathcal{P}}(\xi) / e_{\mathcal{P}}}=\mathrm{N}(\mathcal{P})^{-\operatorname{ord}_{\mathcal{P}}(\xi) / e_{\mathcal{P}} f_{\mathcal{P}}}
$$

for $\xi \in K^{*}$. Here $e_{\mathcal{P}}$ denotes the ramification index of $\mathcal{P}$, $\operatorname{ord}_{\mathcal{P}}(\xi)$ the order of $\mathcal{P}$ in the factorization of $\xi$, and $\mathrm{N}(\mathcal{P})$ the norm of the ideal $\mathcal{P}$. Thus $\xi$ lies in $\mathcal{O}_{K}$ if and only if $\log |\xi|_{v} \leq 0$ for every non-archimedean absolute value $v$.

We denote by $K_{v}$ the completion of $K$ in $\mathbb{C}_{v_{0}}$. The local degree of $K$ at $v$ is defined as:

$$
N_{v}:=\left[K_{v}: \mathbb{Q}_{v_{0}}\right]
$$

and it coincides with the number of different immersions $\sigma: K \hookrightarrow \mathbb{C}_{v_{0}}$ which correspond to $v$. When $v$ is archimedean, $K_{v}$ is either $\mathbb{R}$ or $\mathbb{C}$, and $N_{v}$ equals 1 or 2 accordingly. In the non-archimedean case we have

$$
N_{v}=e_{\mathcal{P}} f_{\mathcal{P}}
$$

where $f_{\mathcal{P}}:=\left[\mathcal{O}_{K} / \mathcal{P}: \mathbb{Z} /(p)\right]$ denotes the residual degree of the prime ideal $\mathcal{P}$ which corresponds to $v$.

We have

$$
[K: \mathbb{Q}]=\sum_{v \mid v_{0}} N_{v}
$$

for $v_{0} \in M_{Q}$. The canonical set $M_{K}$ satisfies the product formula with multiplicities $N_{v}$ :

$$
\prod_{v \in M_{K}}|x|_{v}^{N_{v}}=1, \quad \forall x \in K^{*}
$$

Let $\mathcal{A} \subset K$ be a finite set. Let $v \in M_{K}$ be an absolute value which extends $v_{0} \in M_{Q}$, and let $\sigma_{v}$ be an immersion corresponding to $v$. The local absolute value and the local height of $\mathcal{A}$ at $v$ are defined as

$$
\begin{aligned}
|\mathcal{A}|_{v} & :=\left|\sigma_{v}(\mathcal{A})\right|_{v_{0}}, \\
h_{v}(\mathcal{A}) & :=h_{v_{0}}\left(\sigma_{v}(\mathcal{A})\right),
\end{aligned}
$$

respectively. For a polynomial $f=\sum_{\alpha} a_{\alpha} x^{\alpha} \in K\left[x_{1}, \ldots, x_{n}\right]$, we define the local absolute value of $f$ at $v$ (denoted by $|f|_{v}$ ) as the absolute value at $v$ of its set of coefficients, and the local height of $f$ at $v$ (denoted by $h_{v}(f)$ ) as the logarithmic height at $v$ of its set of coefficients.

Finally the (global) height of a finite set $\mathcal{A} \subset K$ is defined as

$$
h(\mathcal{A}):=\frac{1}{[K: \mathbb{Q}]} \sum_{v \in M_{K}} N_{v} h_{v}(\mathcal{A})
$$

In the same way, the (global) height of $f_{1}, \ldots, f_{s} \in K\left[x_{1}, \ldots, x_{n}\right]$ is defined as the global height of its set of coefficients, that is

$$
h\left(f_{1}, \ldots, f_{s}\right):=\frac{1}{[K: \mathbb{Q}]} \sum_{v \in M_{K}} N_{v} \max _{i} h_{v}\left(f_{i}\right) .
$$

These quantities do not depend on the field $K$ in which we consider the set $\mathcal{A}$ or the polynomials $f_{1}, \ldots, f_{s}$. This allows us to extend the definition of $h$ to the polynomial $\operatorname{ring} \overline{\mathbb{Q}}\left[x_{1}, \ldots, x_{n}\right]$.

We have $h(\mathcal{A}) \geq 0$ and $\max _{a \in \mathcal{A}} h(a) \leq h(\mathcal{A})$. In case $\mathcal{A} \subset \mathcal{O}_{K}, h_{v}(a)=0$ for every $a \in \mathcal{A}$ and $v \notin M_{K}^{\infty}$, and so $h(\mathcal{A}) \leq[K: \mathbb{Q}] \max _{a \in \mathcal{A}} h(a)$. 
Let $q=m / n \in \mathbb{Q}^{*}$ be a rational number, where $m \in \mathbb{Z}$ and $n \in \mathbb{N}$ are coprime. Then $h(q)=\max \{|m|, n\}$, that is, the height of $q$ controls both the size of the minimal numerator and denominator of $q$. More generally, let $\mathcal{A} \subset \mathbb{Q}$ be a finite set, and let $a \in \mathbb{N}$ be a minimal common denominator for all the elements of $\mathcal{A}$. Then $h(\mathcal{A})=\log \max \{|a \mathcal{A}|, a\}$. The following is the analogous statement for the general case:

Lemma 1.3 Let $\mathcal{A} \subset K$ be a finite set. Then there exist $b \in \mathbb{Z} \backslash\{0\}$ and $\mathcal{B} \subset \mathcal{O}_{K}$ such that

$$
b \mathcal{A}=\mathcal{B} \quad, \quad h(\mathcal{A}) \leq h(\{b\} \cup \mathcal{B}) \leq[K: \mathbb{Q}] h(\mathcal{A})
$$

Proof.- Let $v$ be a non-archimedean absolute value, and set $\mathcal{P}$ for the corresponding prime ideal of $\mathcal{O}_{K}$. Then $h_{v}(\mathcal{A})=c(\mathcal{P}) \log \mathrm{N}(\mathcal{P}) / e(\mathcal{P}) f(\mathcal{P})$ for some $c(\mathcal{P}) \geq 0$. We set

$$
b:=\prod_{\mathcal{P}} \mathrm{N}(\mathcal{P})^{c(\mathcal{P})} \quad, \quad \mathcal{B}:=\{b a: a \in \mathcal{A}\} .
$$

Clearly $b \in \mathbb{Z}$, and $\log |b a|_{v} \leq h_{v}(\mathcal{A})-c(\mathcal{P}) \log \mathrm{N}(\mathcal{P}) \leq 0$ for every $v \notin M_{K}^{\infty}$, that is $\mathcal{B} \subset \mathcal{O}_{K}$. We have

$$
h_{v}(b)=\sum_{\mathcal{P}} c(\mathcal{P}) \log \mathrm{N}(\mathcal{P})=\sum_{v \notin M_{K}^{\infty}} N_{v} h_{v}(\mathcal{A})
$$

for $v \in M_{K}^{\infty}$, and also $h_{v}(\mathcal{A})+\log |b|_{v} \leq h_{v}(\{b\} \cup \mathcal{B}) \leq h_{v}(\mathcal{A})+h_{v}(b)$ for all $v \in M_{K}$. Thus

$$
\begin{aligned}
h(\{b\} \cup \mathcal{B}) & \leq \frac{1}{[K: \mathbb{Q}]} \sum_{v \in M_{K}^{\infty}} N_{v}\left(h_{v}(\mathcal{A})+h_{v}(b)\right) \\
& +\frac{1}{[K: \mathbb{Q}]} \sum_{v \in M_{K}^{\infty}} N_{v} h_{v}(\mathcal{A})+\sum_{v \notin M_{K}^{\infty}} N_{v} h_{v}(\mathcal{A}) \\
& \leq[K: \mathbb{Q}] h(\mathcal{A}) .
\end{aligned}
$$

On the other hand

$$
h(\mathcal{A})=\frac{1}{[K: \mathbb{Q}]} \sum_{v} N_{v}\left(h_{v}(\mathcal{A})+\log |b|_{v}\right) \leq \frac{1}{[K: \mathbb{Q}]} \sum_{v} N_{v} h_{v}(\{b\} \cup \mathcal{B})=h(\{b\} \cup \mathcal{B}) .
$$

Finally, let $\alpha \in \overline{\mathbb{Q}}^{*}$ be a non-zero algebraic number, and set $p_{\alpha} \in \mathbb{Z}[t]$ for its primitive minimal polynomial. We have $h(\alpha)=m\left(p_{\alpha}\right) / \operatorname{deg} \alpha$. More generally, the height of a finite set can be seen as the height of the minimal polynomial of a generic linear combination of its elements. This gives a partial motivation for the notion of global height of a finite set.

Lemma 1.4 Let $\mathcal{A}:=\left\{a_{1}, \ldots, a_{N}\right\} \subset K$ be a finite set and set

$$
p_{\mathcal{A}}:=\prod_{\sigma}\left(u_{0}+\sigma\left(a_{1}\right) u_{1}+\cdots+\sigma\left(a_{N}\right) u_{N}\right) \in \mathbb{Q}\left[u_{0}, \ldots, u_{N}\right]
$$

where the product is taken over all $\mathbb{Q}$-immersions $\sigma: K \hookrightarrow \overline{\mathbb{Q}}$. Then

$$
-\log (N+1) \leq h(\mathcal{A})-h\left(p_{\mathcal{A}}\right) /[K: \mathbb{Q}] \leq \log (N+1) .
$$

Proof.- Set $L(u):=u_{0}+a_{1} u_{1}+\cdots+a_{N} u_{N} \in K[u]$. We have $\log |L|_{v}=h_{v}(\mathcal{A})$ and so

$$
h\left(p_{\mathcal{A}}\right) \leq[K: \mathbb{Q}](h(\mathcal{A})+\log (N+1))
$$


by Lemma 1.2(b). On the other hand we have $\log |L|_{v} \leq m\left(\sigma_{v}(L)\right)$ for $v \in M_{K}^{\infty}$ and thus

$$
\begin{aligned}
{[K: \mathbb{Q}] h(\mathcal{A}) } & =\sum_{v \in M_{K}^{\infty}} N_{v} h_{v}(\mathcal{A})+\sum_{v \notin M_{K}^{\infty}} N_{v} h_{v}(\mathcal{A}) \\
& \leq m\left(p_{\mathcal{A}}\right)+\sum_{v \notin M_{K}^{\infty}} N_{v} h_{v}(\mathcal{A}) \\
& \leq h\left(p_{\mathcal{A}}\right)+[K: \mathbb{Q}] \log (N+1) .
\end{aligned}
$$

by application of Lemma 1.2(d) and Inequality (1.1).

\subsection{Height of varieties}

In this section we introduce the notions of local and global height of an affine variety defined over a number field. For this aim, we recall the basic facts of the degree and Chow form of varieties. As an important particular case, we study the height of an affine toric variety.

\subsubsection{Degree of varieties}

Let $k$ be an arbitrary field and $V \subset \mathbb{I}^{n}$ be an affine equidimensional variety of dimension $r$. We recall that the degree of $V$ is defined as the number of points in the intersection of $V$ with a generic linear space of dimension $n-r$. This coincides with the sum of the degrees of its irreducible components.

For an arbitrary variety $V \subset \mathbb{A}^{n}$ we set $V=\cup_{i} V_{i}$ for its decomposition into equidimensional varieties. Following Heintz [20], we define the degree of $V$ as

$$
\operatorname{deg} V:=\sum_{i} \operatorname{deg} V_{i}
$$

For $V=\emptyset$ we agree $\operatorname{deg} V:=1$.

This is a positive integer, and we have $\operatorname{deg} V=1$ if and only $V$ is a linear variety.

The degree of a hypersurface equals the degree of any generator of its defining ideal. The degree of a finite variety equals its cardinal.

For a linear morphism $\varphi: \mathbb{I}^{n} \rightarrow \mathbb{I}^{m}$ and a variety $V \subset \mathbb{I A}^{n}$ we have $\operatorname{deg} \overline{\varphi(V)} \leq \operatorname{deg} V$.

The basic aspect of this notion of degree is its behavior with respect to intersections. It verifies the Bézout inequality:

$$
\operatorname{deg}(V \cap W) \leq \operatorname{deg} V \operatorname{deg} W
$$

for $V, W \subset \mathbb{A}^{n}$, without any restriction on the intersection type of $V$ and $W$ [20], [13].

\subsubsection{Normalization of Chow forms}

Let $V \subset \mathbb{A}^{n}$ be an affine equidimensional variety of dimension $r$ defined over a field $k$. Let $f_{V}$ be a Chow form of $V$, that is a Chow form of its projective closure $\bar{V} \subset \mathbb{P}^{n}$. This is a squarefree polynomial over $k$ in $r+1$ groups $U_{0}, \ldots, U_{r}$ of $n+1$ variables each. It is multihomogeneous of degree $D:=\operatorname{deg} V$ in each group of variables, and is uniquely determined up to a scalar factor. In case $V$ is irreducible, $f_{V}$ is an irreducible polynomial, and in the general case of an equidimensional variety, the product of Chow forms of its irreducible components is a Chow form of $V$.

In order to avoid this indeterminacy of $f_{V}$, we are going to fix one of its coefficients under some assumption on the variety $V$. For purpose of reference, we resume it in the following:

Assumption 1.5 We assume that the projection $\pi_{V}: V \rightarrow \mathbb{A}^{r}$ defined by $x \mapsto\left(x_{1}, \ldots, x_{r}\right)$ verifies $\# \pi_{V}^{-1}(0)=\operatorname{deg} V$. 
This assumption implies that $\pi_{V}: V \rightarrow \mathbb{A}^{r}$ is a dominant map of degree $\operatorname{deg} V$, by the theorem of dimension of fibers. Later on, we will prove that in fact the projection $\pi_{V}$ is finite, that is, the variables $x_{1}, \ldots, x_{r}$ are in Noether normal position with respect to $V$ (Lemma 2.13). We remark that the previous condition is satisfied by any variety under a generic linear change of variables.

Each group of variables $U_{i}$ is associated to the coefficients of a generic linear form $L_{i}\left(U_{i}\right):=$ $U_{i 0}+U_{i 1} x_{1}+\cdots+U_{i n} x_{n}$. The main feature of a Chow form is that

$$
f_{V}\left(\nu_{0}, \ldots, \nu_{r}\right)=0 \Leftrightarrow \bar{V} \cap\left\{L^{h}\left(\nu_{0}\right)=0\right\} \cap \ldots \cap\left\{L^{h}\left(\nu_{r}\right)=0\right\} \neq \emptyset
$$

holds for $\nu_{i} \in \bar{k}^{n+1}$. Here $L_{i}^{h}:=U_{i 0} x_{0}+\cdots+U_{i n} x_{n}$ stands for the homogenization of $L_{i}$.

Assumption 1.5 implies that $\bar{V} \cap\left\{x_{1}=0\right\} \cap \ldots \cap\left\{x_{r}=0\right\}$ is a zero-dimensional variety of $\mathbb{P}^{n}$ lying in the affine space $\left\{x_{0} \neq 0\right\}$. Set $e_{i}$ for the the $(i+1)$-vector of the canonical basis of $k^{n+1}$. Then $f_{V}\left(e_{0}, \ldots, e_{r}\right)$ - that is, the coefficient of the monomial $U_{00}^{D} \cdots U_{r r}^{D}$ - is non-zero.

We then define the (normalized) Chow form $\mathcal{C} h_{V}$ of $V$ by fixing the election of $f_{V}$ through the condition

$$
\mathcal{C} h_{V}\left(e_{0}, \ldots, e_{r}\right)=1 \text {. }
$$

Under this normalization, $\mathcal{C} h_{V}$ equals the product of the normalized Chow forms of the irreducible components of $V$.

\subsubsection{Height of varieties over $\mathbb{C}_{v}$}

Let $v$ be an absolute value over $\mathbb{Q}$, and $V \subset \mathbb{A}^{n}\left(\mathbb{C}_{v}\right)$ an equidimensional variety of dimension $r$ which satisfies Assumption 1.5. We introduce the height of $V$ as a Mahler measure of its normalized Chow form. This notion is closely related to Philippon local height of a projective variety 46, II].

Definition 1.6 The height of the affine variety $V \subset \mathbb{A}^{n}\left(\mathbb{C}_{v}\right)$ is defined as

$$
h_{\infty}(V):=m\left(\mathcal{C} h_{V} ; S_{n+1}^{r+1}\right)+(r+1)\left(\sum_{i=1}^{n} 1 / 2 i\right) \operatorname{deg} V
$$

in case $v=\infty$ is archimedean, and as

$$
h_{p}(V):=h_{p}\left(\mathcal{C} h_{V}\right)
$$

in case $v$ is non-archimedean, that is $v=p$ for some prime $p$.

Let us consider some examples:

- We have that $h_{\infty}\left(\mathbb{A}^{n}\right)$ equals the Stoll number $\sum_{i=1}^{n} \sum_{j=1}^{i} 1 / 2 j$, while $h_{p}\left(\mathbb{A}^{n}\right)=0$. This follows from [5, Lem.3.3.1] and the fact that $\mathcal{C} h_{\mathbb{A}^{n}}=\operatorname{det}\left(U_{0}, \ldots, U_{n}\right)$.

- Let $V \subset \mathbb{A}^{n}\left(\mathbb{C}_{v}\right)$ be a hypersurface verifying Assumption 1.5, defined by a squarefree polynomial $f \in \mathbb{C}_{v}\left[x_{1}, \ldots, x_{n}\right]$. Then the coefficient of the monomial $x_{n}^{\operatorname{deg} V}$ is non-zero, and we can suppose without loss of generality that it equals 1 . Then

$$
\begin{aligned}
h_{\infty}(V) & =m\left(f^{h} ; S_{n+1}\right)+\left(\sum_{i=1}^{n-1} \sum_{j=1}^{i} 1 / 2 j\right) \operatorname{deg} V, \\
h_{p}(V) & =h_{p}(f),
\end{aligned}
$$

where $f^{h}$ denotes the homogenization of $f$ 46, I, Cor. 4].

- In case $V=\{\xi\}$ for some $\xi \in \mathbb{A}^{n}$, we have (see e.g. [46, I, Prop. 4])

$$
\begin{aligned}
h_{\infty}(V) & =\frac{1}{2} \log \left(1+\left|\xi_{1}\right|^{2}+\cdots+\left|\xi_{n}\right|^{2}\right), \\
h_{p}(V) & =h_{p}(\xi) .
\end{aligned}
$$




\subsubsection{Height of varieties over a number field}

Let $V \subset \mathbb{A}^{n}(\overline{\mathbb{Q}})$ be an equidimensional variety of dimension $r$ defined over a number field $K$. We define the (global) height $h(V)$ of $V$ as the Faltings height [10] of its projective closure $\bar{V} \subset \mathbb{P}^{n}$. Following Philippon [46, III], we introduce $h$ - without appealing to Arakelov theory - through the identity

$$
h(V)=\frac{1}{[K: \mathbb{Q}]}\left(\sum_{v \in M_{K}^{\infty}} N_{v} m\left(\sigma_{v}\left(f_{V}\right) ; S_{n+1}^{r+1}\right)+\sum_{v \notin M_{K}^{\infty}} N_{v} \log \left|f_{V}\right|_{v}\right)+(r+1)\left(\sum_{i=1}^{n} 1 / 2 i\right) \operatorname{deg} V,
$$

where $f_{V}$ denotes any Chow form of $V$ [52, 446, I]. This coincides with the sum of the heights of the irreducible components of $V$.

For an arbitrary affine variety, we define its (global) height as the sum of the heights of its equidimensional components. We agree that $h(\emptyset):=0$.

We introduce the local counterpart of this notion. Let $v \in M_{K}$ be absolute value over $K$, and suppose that $V$ satisfies Assumption 1.5. Let $v_{0} \in M_{Q}$ such that $v \mid v_{0}$, and let $\sigma_{v}: K_{v} \rightarrow \mathbb{C}_{v_{0}}$ be an immersion corresponding to $v$. We define the local height of $V$ at $v$ as

$$
h_{v}(V):=h_{v_{0}}\left(\sigma_{v}(V)\right)
$$

This definition is consistent with the global height, namely

$$
h(V)=\frac{1}{[K: \mathbb{Q}]} \sum_{v \in M_{K}} N_{v} h_{v}(V)
$$

This notion is related to the height $\widehat{h}$ of Bost, Gillet and Soulé, by the formula [5, Prop. 4.1.2 (i)]:

$$
h(V)=\widehat{h}(V)+\left(\sum_{i=1}^{r} \sum_{j=1}^{i} 1 / 2 j\right) \operatorname{deg} V .
$$

It is also related to the height $\widetilde{h}$ introduced by Giusti et al. [15] in terms of the so-called geometric solution of a variety. They are polynomially equivalent [51, 1.3.4], namely

$$
h(V) \leq(n \operatorname{deg} V \widetilde{h}(V))^{c} \quad, \quad \widetilde{h}(V) \leq(n \operatorname{deg} V h(V))^{c},
$$

for some constant $c>0$.

We have $h(V) \geq 0$. Moreover $h(V) \geq\left(\sum_{i=1}^{r} \sum_{j=1}^{i} 1 / 2 j\right) \operatorname{deg} V$, with equality only in case $V$ is defined by the vanishing of $n-r$ standard coordinates. [5, Th. 5.2.3]. For instance $h\left(\mathbb{I}^{n}\right)=$ $\sum_{i=1}^{n} \sum_{j=1}^{i} 1 / 2 j$.

This notion of height satisfies the arithmetic Bezout inequality [5, Th. 5.5.1 (iii)], [46, III, Th. 3]:

$$
h(V \cap W) \leq h(V) \operatorname{deg} W+\operatorname{deg} V h(W)+c \operatorname{deg} V \operatorname{deg} W
$$

for $V, W \subset \mathbb{A}^{n}(\overline{\mathbb{Q}})$, with $c:=\left(\sum_{i=0}^{\operatorname{dim} V} \sum_{j=0}^{\operatorname{dim} W} 1 / 2(i+j+1)\right)+(n-(\operatorname{dim} V+\operatorname{dim} W) / 2) \log 2$.

\subsubsection{Height of affine toric varieties}

Now we consider the case of affine toric varieties. The obtained height estimate is crucial in our treatment of the sparse arithmetic Nullstellensatz (Corollary 1.12).

Let $\mathcal{A}=\left\{\alpha_{1}, \ldots, \alpha_{N}\right\} \subset \mathbb{Z}^{n}$ be a finite set of integer vectors such that $0 \in \mathcal{A}$. Let $r:=\operatorname{dim} \mathcal{A}$ denote the dimension of $\mathcal{A}$, that is, the dimension of the free $\mathbb{Z}$-module $\mathbb{Z} \mathcal{A}$. We normalize 
the volume form of $\mathbb{R} \mathcal{A}$ in order that any elementary simplex of the lattice $\mathbb{Z} \mathcal{A}$ has volume 1 . The (normalized) volume $\operatorname{Vol}(\mathcal{A})$ of $\mathcal{A}$ is defined as the volume of the convex hull $\operatorname{Conv}(\mathcal{A})$ with respect to this volume form. In case $\mathbb{Z} \mathcal{A}=\mathbb{Z}^{n}$, then $\operatorname{Vol}(\mathcal{A})$ equals $n$ ! times the volume of $\operatorname{Conv}(\mathcal{A})$ with respect to the Euclidean volume form of $\mathbb{R}^{n}$.

We associate to the set $\mathcal{A}$ a map $\left(\overline{\mathbb{Q}}^{*}\right)^{n} \rightarrow \overline{\mathbb{Q}}^{N}$ defined by $\xi \mapsto\left(\xi^{\alpha_{1}}, \ldots, \xi^{\alpha_{N}}\right)$. The Zariski closure of the image of this map is the affine toric variety $X_{\mathcal{A}} \subset \mathbb{A}^{N}$. This is an irreducible variety of dimension $r$ and degree $\operatorname{Vol}(\mathcal{A})$.

For $i=0, \ldots, r$, let $U_{i}$ denote a group of variables indexed by the elements of $\mathcal{A}$ and set

$$
F_{i}:=\sum_{\alpha \in \mathcal{A}} U_{i \alpha} x^{\alpha}
$$

for the generic Laurent polynomial with support contained in $\mathcal{A}$. Let $W \subset\left(\mathbb{P}^{N-1}\right)^{r+1} \times\left(\overline{\mathbb{Q}}^{*}\right)^{n}$ be the incidence variety of $F_{0}, \ldots, F_{r}$ in $\left(\overline{\mathbb{Q}}^{*}\right)^{n}$, that is

$$
W=\left\{\left(\nu_{0}, \ldots, \nu_{r} ; \xi\right) ; \quad F_{i}\left(\nu_{i}\right)(\xi)=0 \quad \forall i\right\}
$$

and let $\pi:\left(\mathbb{P}^{N-1}\right)^{r+1} \times\left(\overline{\mathbb{Q}}^{*}\right)^{n} \rightarrow\left(\mathbb{P}^{N-1}\right)^{r+1}$ be the canonical projection. Then $\overline{\pi(W)}$ is an irreducible variety of codimension 1 . Its defining polynomial $R_{\mathcal{A}} \subset \mathbb{Q}\left[U_{0}, \ldots, U_{r}\right]$ is called the $\mathcal{A}$-resultant or sparse resultant, and it coincides with the Chow form of the affine toric variety $X_{\mathcal{A}}$ [24]. It is a multihomogeneous polynomial of degree $\operatorname{Vol}(\mathcal{A})$ in each group of variables, and it is uniquely defined up to its sign, if we assume it to be a primitive polynomial with integer coefficients. Basic references for affine toric varieties and sparse resultants are [14], [54].

We obtain the following bound for the height of $X_{\mathcal{A}}$. Our argument relies on the Canny-Emiris determinantal formula for the sparse resultant [8].

Proposition 1.7 Let $\mathcal{A} \subset \mathbb{Z}^{n}$ be a finite set of dimension $r$ and cardinality $\# \mathcal{A} \geq 2$. Then $h\left(X_{\mathcal{A}}\right) \leq 2^{2 r+2} \log (\# \mathcal{A}) \operatorname{Vol}(\mathcal{A})$.

Proof.- Let $\mathcal{M}$ be the Canny-Emiris matrix associated to the generic polynomial system $F_{0}, \ldots, F_{r}$. This is a non-singular square matrix of order $M$, where $M$ denotes the cardinality of the set

$$
\mathcal{E}:=((r+1) Q+\varepsilon) \cap \mathbb{Z}^{n} .
$$

Here $Q:=\operatorname{Conv}(\mathcal{A})$, and $\varepsilon \in \mathbb{R}^{n}$ is a vector such that each point in $\mathcal{E}$ is contained in the interior of a cell in a given triangulation of the polytope $(r+1) Q$.

Every non-zero entry of $\mathcal{M}$ is a variable $U_{i \alpha}$. In fact, each row has exactly $N$ non-zero entries, which consist of the variables in some group $U_{i}$. We refer to [8] for the precise construction.

Thus $\operatorname{det} \mathcal{M} \in \mathbb{Z}\left[U_{0}, \ldots, U_{r}\right]$ is a multihomogeneous polynomial of total degree $M$ and height bounded by $M \log N$. This polynomial is a non-zero multiple of the sparse resultant $R_{\mathcal{A}}$ [8]. The assumption that $R_{\mathcal{A}}$ is primitive implies that $\operatorname{det} \mathcal{M} / R_{\mathcal{A}}$ lies in $\mathbb{Z}\left[U_{0}, \ldots, U_{r}\right]$, and so $m\left(R_{\mathcal{A}}\right) \leq$ $m(\operatorname{det} \mathcal{M})$.

Let $\left\{T_{j}\right\}_{j \in I}$ be a unimodular triangulation of $Q$, so that $\left\{(r+1) T_{j}\right\}_{j \in I}$ is a triangulation of $(r+1) Q$. For every $\varepsilon \in \mathbb{R}^{n}$, the set of integer points in $(r+1) T_{j}+\varepsilon$ is in correspondence with a subset of those of $(r+1) T_{j}$. Moreover, for a generic choice of $\varepsilon$ we loose - at least - the set of integer points in a facet of codimension 1 . Thus

$$
\#\left((r+1) T_{j}+\varepsilon\right) \cap \mathbb{Z}^{n}=\left(\begin{array}{c}
2 r \\
r
\end{array}\right) \leq 2^{2 r}
$$


and so $\quad M \leq 2^{2 r} \operatorname{Vol}(\mathcal{A})$. Applying Lemma 1.1 we obtain

$$
\begin{aligned}
m\left(R_{\mathcal{A}}\right) & \leq \log |\operatorname{det} \mathcal{M}|+\operatorname{deg}(\operatorname{det} \mathcal{M}) \log N \\
& \leq 2 M \log N \\
& \leq 2^{2 r+1} \log N \operatorname{Vol}(\mathcal{A}) .
\end{aligned}
$$

We conclude

$$
\begin{aligned}
h\left(X_{\mathcal{A}}\right) & =m\left(R_{\mathcal{A}} ; S_{N+1}^{r+1}\right)+(r+1)\left(\sum_{i=1}^{N} 1 / 2 i\right) \operatorname{Vol}(\mathcal{A}) \\
& \leq m\left(R_{\mathcal{A}}\right)+2(r+1) \log N \operatorname{Vol}(\mathcal{A}) \\
& \leq 2^{2 r+2} \log N \operatorname{Vol}(\mathcal{A}),
\end{aligned}
$$

as $N=\# \mathcal{A} \geq 2$.

In case $\mathcal{A} \subset\left(\mathbb{Z}_{\geq 0}\right)^{n}$ - that is, when $F_{0}, \ldots, F_{r}$ are polynomials - we set $d:=\max \{|\alpha|: \alpha \in$ $\mathcal{A}\}=\operatorname{deg} F_{0}$. We have then $N \leq(n+1)^{d}$ and so

$$
h\left(X_{\mathcal{A}}\right) \leq 2^{2 r+2} \log (n+1) d \operatorname{Vol}(\mathcal{A}) .
$$

\section{Estimates for local and global heights}

In this chapter we study the basic properties of local and global heights that we will need for our purposes. The key result is a precise estimate for the local height of the trace and the norm of a polynomial $f \in K\left[x_{1}, \ldots, x_{n}\right]$ with respect to an integral extension $K\left[\mathbb{A}^{r}\right] \hookrightarrow K[V]$.

We also study some of the basic properties of the height of a variety, in particular its behavior under intersection with hypersurfaces and under affine maps.

\subsection{Estimates for Chow forms}

In this section we recall the notion of generalized Chow form of a variety in the sense of Philippon [14], and we prove a technical estimate for its local height.

\subsubsection{Generalized Chow forms}

Let $V \subset \mathbb{I}^{n}$ be an affine equidimensional variety of dimension $r$ and degree $D$ defined over a field $k$.

For $d \in \mathbb{N}$ we denote by $U(d)_{0}$ a group of $\left(\begin{array}{c}d+n \\ n\end{array}\right)$ variables. Also, for $1 \leq i \leq r$ we denote by $U_{i}$ a group of $n+1$ variables, and we set $U(d):=\left\{U(d)_{0}, U_{1}, \ldots, U_{r}\right\}$. Set

$$
F:=\sum_{|\alpha| \leq d} U(d)_{0 \alpha} x^{\alpha} \quad, \quad L_{i}:=U_{i 0}+U_{i 1} x_{1}+\cdots+U_{i n} x_{n}
$$

for the generic polynomial in $n$ variables of degree $d$ and 1 associated to $U(d)_{0}$ and $U_{i}$ respectively.

Set $N:=\left(\begin{array}{c}d+n \\ n\end{array}\right)+r(n+1)$ and let $W \subset \mathbb{I}^{N} \times V$ be the incidence variety of $F, L_{1}, \ldots, L_{r}$ with respect to $V$, that is

$$
W:=\left\{\left(\nu(d)_{0}, \nu_{1}, \ldots, \nu_{r} ; \xi\right) ; \quad \xi \in V, \quad F\left(\nu(d)_{0}\right)(\xi)=0, \quad L_{i}\left(\nu_{i}\right)(\xi)=0,1 \leq i \leq r\right\} .
$$


Let $\pi: \mathbb{A}^{N} \times \mathbb{A}^{n} \rightarrow \mathbb{A}^{N}$ denote the canonical projection. Then $\overline{\pi(W)} \subset \mathbb{I}^{N}$ is a hypersurface [14. Prop. 1.5] and any of its defining equations $f_{d, V} \in k[U(d)]$ is called a generalized Chow form or a $d$-Chow form of $V$.

A $d$-Chow form is uniquely defined up to a scalar factor. It shares many properties with the usual Chow form, which corresponds to the case $d=1$. We have

$$
f_{d, V}\left(\nu(d)_{0}, \nu_{1}, \ldots, \nu_{r}\right)=0 \Leftrightarrow \bar{V} \cap\left\{F^{h}\left(\nu(d)_{0}\right)=0\right\} \cap\left\{L_{1}^{h}\left(\nu_{1}\right)=0\right\} \cap \cdots \cap\left\{L_{r}^{h}\left(\nu_{r}\right)=0\right\} \neq \emptyset
$$

for $\nu(d)_{0} \in \bar{k}^{\left(\begin{array}{c}d+n \\ n\end{array}\right)}$ and $\nu_{i} \in \bar{k}^{n+1}$. Here $\bar{V} \subset \mathbb{P}^{n}$ denotes the projective closure of $V$, while $F^{h}$ and $L_{i}^{h}$ stand for the homogenization of $F$ and $L_{i}$ respectively.

A $d$-Chow form $f_{d, V} \in k[U(d)]$ is a multihomogeneous polynomial of degree $D$ in the group of variables $U(d)_{0}$ and of degree $d D$ in each group $U_{i}$ [44, Lem. 1.8]. When $V$ is an irreducible variety, $f_{d, V}$ is an irreducible polynomial of $k[U(d)]$. When $V$ is equidimensional, it coincides with the product of $d$-Chow forms of its irreducible components.

We will appeal to the following relation between a $d$-Chow form $f_{d, V}$ and the usual one: Let $U_{0}$ be another group of $n+1$ variables, and consider the morphism

$$
\varrho_{d}: k[U(d)] \rightarrow k\left[U_{0}, U_{1}, \ldots, U_{r}\right]
$$

defined by $\varrho_{d}(F)=L_{0}^{d}$ and $\varrho_{d}\left(L_{i}\right)=L_{i}$ for $i=1, \ldots, r$, where $L_{0}$ stands for the generic linear form associated to $U_{0}$. In other terms

$$
\varrho_{d}\left(U(d)_{0 \alpha}\right)=\left(\begin{array}{l}
d \\
\alpha
\end{array}\right) U_{00}^{d-|\alpha|} U_{01}^{\alpha_{1}} \cdots U_{0 n}^{\alpha_{n}} \quad \text { where }\left(\begin{array}{c}
d \\
\alpha
\end{array}\right):=\frac{d !}{(d-|\alpha|) ! \alpha_{1} ! \cdots \alpha_{n} !}
$$

for $|\alpha| \leq d$, and $\varrho_{d}\left(U_{i j}\right)=U_{i j}$ for $i=1, \ldots, r$ and $j=0, \ldots, n$. Then $\varrho_{d}\left(f_{d, V}\right)=\lambda f_{V}^{d}$ for some $\lambda \in k^{*}$ [4. Prop. 1.4].

Now assume that $V$ satisfies Assumption 1.5. Then $\bar{V} \cap\left\{x_{0}^{d}=0\right\} \cap\left\{x_{1}=0\right\} \cap \ldots \cap\left\{x_{r}=0\right\}=\emptyset$. Setting $e(d)_{\alpha}$ and $e_{i}$ for the $\alpha$-vector and the $(i+1)$-vector of the canonical bases of $k^{\left(\begin{array}{c}d+n \\ n\end{array}\right)}$ and $k^{n+1}$ respectively, we infer that $f_{d, V}\left(e(d)_{0}, e_{1}, \ldots, e_{r}\right)$ - that is, the coefficient of the monomial $U(d)_{00}^{D} U_{11}^{d D} \cdots U_{r r}^{d D}$ - is non-zero.

We define the (normalized) $d$-Chow form $\mathcal{C} h_{d, V}$ of $V$ by fixing the election of $f_{d, V}$ with the condition $\mathcal{C} h_{V}\left(e(d)_{0}, e_{1}, \ldots, e_{r}\right)=1$.

In the previous construction, $U(d)_{00}^{D} U_{11}^{d D} \ldots U_{r r}^{d D}$ is the only monomial of $k[U(d)]$ which maps through $\varrho_{d}$ to $U_{00}^{d D} \cdots U_{r r}^{d D}$. The imposed normalizations imply then

$$
\varrho_{d}\left(\mathcal{C} h_{d, V}\right)=\mathcal{C} h_{V}^{d} .
$$

\subsubsection{An estimate for generalized Chow forms}

The following technical result is crucial to our local height estimates for the trace and the norm of a polynomial (Subsection 2.3.2), as well as for the intersection of a variety with an hypersurface (Subsection 2.2.2). The proof follows the lines of [44, Prop. 2.8].

We adopt the following convention:

Let $f \in k\left[x_{1}, \ldots, x_{n}\right]$ be a polynomial of degree $d$. We denote by $f_{d, V}(f)$ and $\mathcal{C} h_{d, V}(f)$ the specialization of $U(d)_{0}$ into the coefficients of $f$ in $f_{d, V}$ and $\mathcal{C} h_{d, V}$ respectively.

Lemma 2.1 Let $V \subset \mathbb{A}^{n}\left(\mathbb{C}_{v}\right)$ be an equidimensional variety of dimension $r$ which satisfies Assumption 1.9. Let $f \in \mathbb{C}_{v}\left[x_{1}, \ldots, x_{n}\right]$. Then 
- $m\left(\mathcal{C} h_{\operatorname{deg} f, V}(f) ; S_{n+1}^{r}\right)+r\left(\sum_{i=1}^{n} 1 / 2 i\right) \operatorname{deg} f \operatorname{deg} V$

if $v=\infty$,

$\leq \operatorname{deg} f h_{\infty}(V)+h_{\infty}(f) \operatorname{deg} V+\log (n+1) \operatorname{deg} f \operatorname{deg} V$

- $h_{p}\left(\mathcal{C} h_{\operatorname{deg} f, V}(f)\right) \leq \operatorname{deg} f h_{p}(V)+h_{p}(f) \operatorname{deg} V \quad$ if $v=p$ for some prime $p$.

We will need the following lemma in order to treat the non-archimedean case:

Lemma 2.2 Let $f \in \mathbb{C}_{p}\left[x_{1}, \ldots, x_{n}\right]$, and let $\Omega \subset \mathbb{A}^{n}\left(\mathbb{C}_{p}\right)$ be a Zariski open set. Then

$$
|f|_{p}=\max \left\{|f(z)|_{p} ; \quad z \in \Omega,|z|_{p}=1\right\} .
$$

Proof.- For $q \in \mathbb{N}$ we denote by $G_{q}$ the set of $q$-roots of 1 in $\overline{\mathbb{Q}} \subset \mathbb{C}_{p}$. Let $\alpha=\left(\alpha_{1}, \ldots, \alpha_{n}\right) \in \mathbb{Z}^{n}$ such that $\left|\alpha_{i}\right|<q$. Then

$$
\sum_{\omega \in G_{q}^{n}} \omega^{\alpha}=\left\{\begin{array}{lll}
0 & \text { if } & \alpha \neq 0, \\
q^{n} & \text { if } & \alpha=0 .
\end{array}\right.
$$

Set $f=\sum_{\alpha} a_{\alpha} x^{\alpha}$. Let $q>\operatorname{deg} f$ such that $|q|_{p}=1$, that is $p \bigvee q$. Then for any $\nu=\left(\nu_{1}, \ldots, \nu_{n}\right) \in$ $\left(\mathbb{C}_{p}^{*}\right)^{n}$ we have

$$
a_{\alpha}=\frac{1}{\nu^{\alpha} q^{n}} \sum_{\omega \in G_{q}^{n}} f(\nu \omega) \omega^{-\alpha} .
$$

Let $\alpha \in\left(\mathbb{Z}_{\geq 0}\right)^{n}$ such that $|f|_{p}=\left|a_{\alpha}\right|_{p}$. From the previous expression we derive that for each $\nu \in S_{n}:=\left\{\nu ;\left|\nu_{i}\right|_{p}=1\right\}$ there exists $\omega_{0} \in G_{q}^{n}$ such that $|f|_{p}=\left|f\left(\nu \omega_{0}\right)\right|_{p}$. The set $S_{n}$ is Zariski dense in $I \mathrm{~A}^{n}\left(\mathbb{C}_{p}\right)$, and so $S_{n} \cap \Omega$ is also dense. Thus we can take $\nu$ such that $\nu G_{q} \subset \Omega$, and therefore

$$
|f|_{p} \leq \max \left\{|f(z)|_{p} ; z \in \Omega,|z|_{p}=1\right\} .
$$

The other inequality is straightforward.

Proof of Lemma 2.1.- First we consider the case when $V$ is a 0 -dimensional variety. We may assume without loss of generality that $V$ is irreducible, that is $V=\{\xi\}$ for some $\xi=\left(\xi_{1}, \ldots, \xi_{n}\right) \in$ $\mathbb{C}_{v}^{n}$

Set $d:=\operatorname{deg} f$. Then

$$
\mathcal{C} h_{V}=L(\xi):=U_{0}+U_{1} \xi_{1}+\cdots+U_{n} \xi_{n} \quad, \quad \mathcal{C} h_{d, V}=F(\xi):=\sum_{\alpha} U_{\alpha} \xi^{\alpha}
$$

where $L$ and $F$ denote generic polynomials in $n$ variables of degree 1 and $d$ respectively. Then

$$
\begin{aligned}
h_{\infty}(F(\xi)) & =\log \max _{|\alpha| \leq d}\left\{\left|\xi^{\alpha}\right|\right\} \\
& =\log \max _{i}\left\{1,\left|\xi_{i}\right|^{d}\right\} \\
& =d h_{\infty}(L(\xi)) \\
& \leq d m\left(L(\xi) ; S_{n+1}\right)+\left(\sum_{i=1}^{n} 1 / 2 i\right) d
\end{aligned}
$$

and so $h_{\infty}\left(\mathcal{C} h_{d, V}(f)\right) \leq d h_{\infty}(F(\xi))+h_{\infty}(f)+\log (n+1) d \leq d h_{\infty}(V)+h_{\infty}(f)+\log (n+1) d$. Analogously, $h_{p}(F(\xi)) \leq d h_{p}(L(\xi))$ and so $h_{p}\left(\mathcal{C} h_{d, V}(f)\right) \leq d h_{p}(V)+h_{p}(f)$.

Now we consider the general case. Set $\nu=\left(\nu_{1}, \ldots, \nu_{r}\right) \in \mathbb{C}_{v}^{r(n+1)}, L\left(\nu_{i}\right):=\nu_{i 0}+\nu_{i 1} x_{1}+\cdots+\nu_{i n} x_{n}$ and

$$
V(\nu):=V \cap V\left(L\left(\nu_{1}\right), \ldots, L\left(\nu_{r}\right)\right) \subset \mathbb{A}^{n}\left(\mathbb{C}_{v}\right)
$$


Then $V(\nu)$ is a 0 -dimensional variety of degree $\operatorname{deg} V$ for $\nu$ in a Zariski open set $\Omega$ of $\operatorname{IA}^{r(n+1)}\left(\mathbb{C}_{v}\right)$. Let $\nu \in \Omega$. By 44. Prop. 2.4] there exist $\lambda(\nu), \theta(\nu) \in k^{*}$ such that

$$
\mathcal{C} h_{V(\nu)}=\lambda(\nu) \mathcal{C} h_{V}(\nu) \quad, \quad \mathcal{C} h_{d, V(\nu)}=\theta(\nu) \mathcal{C} h_{d, V}(\nu),
$$

where $\mathcal{C} h_{V}(\nu), \mathcal{C} h_{d, V}(\nu)$ stand for the specialization of $U_{1}, \ldots, U_{r}$ into $\nu_{1}, \ldots, \nu_{r}$. Applying the morphism $\varrho_{d}$ linking the $d$-Chow form with the usual one we obtain

$$
\mathcal{C} h_{V(\nu)}^{d}=\varrho_{d}\left(\mathcal{C} h_{d, V(\nu)}\right)=\theta(\nu) \varrho_{d}\left(\mathcal{C} h_{d, V}(\nu)\right)=\theta(\nu) \mathcal{C} h_{V}^{d}(\nu)
$$

and so $\theta(\nu)=\lambda(\nu)^{d}$.

We consider the case $v=\infty$. Any Zariski closed set of $I^{r(n+1)}(\mathbb{C})$ intersects $S_{n+1}^{r}$ in a set of $\mu_{n+1}^{r}$-measure 0 , and so the previous relation holds for almost every $\nu \in S_{n+1}^{r}$. Therefore

$$
\begin{aligned}
m\left(\mathcal{C} h_{d, V}(f) ; S_{n+1}^{r}\right)= & \int_{S_{n+1}^{r}}\left(\log \left|\mathcal{C} h_{d, V(\nu)}(f)\right|-d \log |\lambda(\nu)|\right) \mu_{n+1}^{r} \\
\leq & \int_{S_{n+1}^{r}}\left(d h_{\infty}(V(\nu))+h_{\infty}(f) \operatorname{deg} V(\nu)+\log (n+1) d \operatorname{deg} V(\nu)-d \log |\lambda(\nu)|\right) \mu_{n+1}^{r} \\
= & d \int_{S_{n+1}^{r}} m\left(\mathcal{C} h_{V}(\nu) ; S_{n+1}\right) \mu_{n+1}^{r}+\left(\sum_{i=1}^{n} 1 / 2 i\right) d \operatorname{deg} V+h_{\infty}(f) \operatorname{deg} V \\
& +\log (n+1) d \operatorname{deg} V \\
= & d h_{\infty}(V)+h_{\infty}(f) \operatorname{deg} V+\log (n+1) d \operatorname{deg} V-r\left(\sum_{i=1}^{n} 1 / 2 i\right) d \operatorname{deg} V .
\end{aligned}
$$

The case $v=p$ follows analogously from the 0 -dimensional case and the previous lemma.

Remark 2.3 The only role played by Assumption 1.5 in the proof of the previous result is in the normalization of the involved Chow forms. This is essential in order to properly define local heights of varieties. If we disregard normalization, we get altogether the following global result:

Let $V \subset \mathbb{A}^{n}$ be an equidimensional variety of dimension $r$ defined over a number field $K$, and let $f_{d, V}$ be a $d$-Chow form of $V$. Let $f \in K\left[x_{1}, \ldots, x_{n}\right]$ be a polynomial of degree $d$. Then

$\frac{1}{[K: \mathbb{Q}]}\left(\sum_{v \in M_{K}^{\infty}} N_{v} m\left(\sigma_{v}\left(f_{d, V}(f)\right) ; S_{n+1}^{r}\right)+\sum_{v \notin M_{K}^{\infty}} N_{v} \log \left|f_{d, V}(f)\right|_{v}\right)+r\left(\sum_{i=1}^{n} 1 / 2 i\right) d \operatorname{deg} V \leq$

$$
d h(V)+h(f) \operatorname{deg} V+\log (n+1) d \operatorname{deg} V .
$$

\subsection{Basic properties of the height}

We derive some of the basic properties of the notion of height of a variety. In particular, we study the behavior of the height of a variety under intersection with a hypersurface and under an affine map.

We also obtain an arithmetic version of the Bernstein-Kushnirenko theorem.

\subsubsection{Height of varieties under affine maps}

Let $\varphi: \mathbb{A}^{n} \rightarrow \mathbb{A}^{m}$ be a regular map defined by polynomials $\varphi_{1}, \ldots, \varphi_{m} \in K\left[x_{1}, \ldots, x_{n}\right]$. We recall that the height of $\varphi$ is defined as $h(\varphi):=h\left(\varphi_{1}, \ldots, \varphi_{m}\right)$.

We obtain the following estimate for the height of the image of a variety under an affine map: 
Proposition 2.4 Let $V \subset \mathbb{A ^ { n }}$ be a variety of dimension $r$, and let $\varphi: \mathbb{A}^{n} \rightarrow I^{N}$ be an affine map. Then

$$
h(\varphi(V)) \leq h(V)+(r+1)(h(\varphi)+8 \log (n+N+1)) \operatorname{deg} V .
$$

The proof of this result follows from the study of the particular cases of a linear projection and an injective affine map.

The following estimate for the height of a linear projection of a variety generalizes [10, Prop. 2.10] and $[5,3.3 .2]$. Its proof is essentially based on the description of the Chow form of such projection variety, due to Pedersen and Sturmfels [43, Prop. 4.1].

Lemma 2.5 Let $V \subset \mathbb{A}^{n} \times \mathbb{A}^{m}$ be a variety of dimension $r$, and let $\pi: \mathbb{A}^{n} \times \mathbb{A}^{m} \rightarrow \mathbb{A}^{n}$ denote the projection $(x, y) \mapsto x$. Then

$$
h(\overline{\pi(V)}) \leq h(V)+3(r+1) \log (n+m+1) \operatorname{deg} V .
$$

Proof. We assume without loss of generality that $V$ is irreducible. Set $W:=\overline{\pi(V)} \subset \mathbb{I}^{n}$ and $s:=\operatorname{dim} W$.

The case $s=r$ follows directly from [43, Prop. 4.1]: in this case, there exists a partial monomial order $\prec$ such that

$$
f_{W} \mid \operatorname{init} f_{V}
$$

where $\operatorname{init} f_{V}$ denotes the initial polynomial of $f_{V}$ with respect to $\prec$. In particular $\operatorname{init} f_{V}$ is the sum of some of the terms in the monomial expansion of $f_{V}$.

The general case $s \leq r$ reduces to the previous one: we choose standard coordinates $z_{s+1}, \ldots, z_{r}$ of $I^{m}$ such that the projection

$$
\varpi: \mathbb{A}^{n} \times \mathbb{A}^{m} \rightarrow \mathbb{A}^{n} \times \mathbb{A}^{r-s} \quad, \quad(x, y) \mapsto(x, z)
$$

verifies $\operatorname{dim} Z=r$ for $Z:=\overline{\varpi(V)}$.

Let $\varrho: \mathbb{A}^{n} \times \mathbb{A}^{r-s} \rightarrow \mathbb{A}^{n}$ denote the canonical projection. Then $f_{Z} \mid \operatorname{init} f_{V}, \pi=\varrho \circ \varpi$ and $W=\overline{\varrho(Z)}$. We have that $\varrho^{-1}(\xi)=\{\xi\} \times \mathbb{I}^{r-s}$ for $\xi \in \varrho(Z)$ by the theorem of dimension of fibers. Thus $Z=W \times \mathbb{A}^{r-s}$, and in particular

$$
i(W)=Z \cap V\left(z_{s+1}, \ldots, z_{r}\right) \subset \mathbb{A}^{n} \times \mathbb{A}^{r-s},
$$

where $i$ denotes the canonical inclusion $I^{n} \hookrightarrow I^{n} \times I A^{r-s}$. We have $\operatorname{deg} W=\operatorname{deg} Z$ and so $f_{W}:=f_{Z}\left(z_{s+1}, \ldots, z_{r}\right)$ is a Chow form of $W$ 44, Prop. 2.4].

Now we estimate the height of $f_{W}$. Let $K$ be a number field of definition of $V$, and set

$$
\operatorname{init} f_{V}=Q f_{Z}
$$

for some polynomial $Q$. From the proof of [44, Lem. 1.12(v)], there is a non-zero coefficient $\lambda$ of $Q$ such that $\log |\lambda|_{v} \leq m\left(\sigma_{v}(Q)\right)$ for all $v \in M_{K}^{\infty}$. Clearly $\log |\lambda|_{v} \leq \log |Q|_{v}$ also holds for all $v \notin M_{K}^{\infty}$. Thus

$$
m\left(\sigma_{v}\left(f_{Z}\right)\right) \leq m\left(\sigma_{v}\left(\text { init } f_{V}\right)\right)-\log |\lambda|_{v}
$$

for $v \in M_{K}^{\infty}$, while $\log \left|f_{Z}\right|_{v} \leq \log \left|\operatorname{init} f_{V}\right|_{v}-\log |\lambda|_{v}$ for $v \notin M_{K}^{\infty}$.

Let $v \in M_{K}^{\infty}$. From [44, Lem. 1.13] we obtain $m\left(\sigma_{v}\left(f_{W}\right)\right) \leq m\left(\sigma_{v}\left(f_{Z}\right)\right)$. Hence

$$
\begin{aligned}
m\left(\sigma_{v}\left(f_{W}\right) ; S_{n+1}^{s+1}\right) \leq & m\left(\sigma_{v}\left(f_{W}\right)\right) \\
\leq & m\left(\sigma_{v}\left(\operatorname{init} f_{V}\right)\right)-\log |\lambda|_{v} \\
\leq & \log \left|\operatorname{init} f_{V}\right|_{v}+(r+1) \log (n+m+1) \operatorname{deg} V-\log |\lambda|_{v} \\
\leq & \log \left|f_{V}\right|_{v}+(r+1) \log (n+m+1) \operatorname{deg} V-\log |\lambda|_{v} \\
\leq & m\left(\sigma_{v}\left(f_{V}\right) ; S_{n+m+1}^{r+1}\right)+(r+1)\left(\sum_{i=1}^{n+m} 1 / 2 i\right) \operatorname{deg} V \\
& +2(r+1) \log (n+m+1) \operatorname{deg} V-\log |\lambda|_{v}
\end{aligned}
$$


by application of Lemma 1.1 and Inequality (1.2). In case $v \notin M_{K}^{\infty}$ we have analogously $\log \left|f_{W}\right|_{v} \leq$ $\log \left|f_{V}\right|_{v}-\log |\lambda|_{v}$, and so

$$
\begin{aligned}
h(W) & \leq h(V)+(s+1)\left(\sum_{i=1}^{n} 1 / 2 i\right) \operatorname{deg} V+2(r+1) \log (n+m+1) \operatorname{deg} V \\
& \leq h(V)+3(r+1) \log (n+m+1) \operatorname{deg} V .
\end{aligned}
$$

The following is a variant of [46, I, Prop. 7]:

Lemma 2.6 Let $V \subset \mathbb{I A}^{m}$ be a variety of dimension $r$, and let $\psi: \mathbb{I A}^{m} \rightarrow I \mathrm{~A}^{n}$ be an injective affine map. Then

$$
h(\psi(V)) \leq h(V)+(r+1)(h(\psi)+5 \log (n+1)) \operatorname{deg} V .
$$

Proof.- We assume again without loss of generality that $V$ is irreducible. Let $K$ be a number field of definition of both $V$ and $\psi$, and set $\psi(x)=a+A x$ for some $m \times n-$ matrix $A$ of maximal rank and $a \in K^{n}$. Then let $\psi^{*}: \mathbb{I}^{n+1} \rightarrow \mathbb{I}^{m+1}$ be the linear map $y \mapsto(a, A)^{t} y$ defined by the transpose of the matrix associated to $\psi$.

Set $W:=\psi(V)$, and let $\bar{V} \subset \mathbb{P}^{m}, \bar{W} \subset \mathbb{P}^{n}$ denote the projective closures of $V$ and $W$ respectively.

For $i=0, \ldots, r$ we let $\nu_{i} \in \overline{\mathbb{Q}}^{n+1}$, and we set $L^{h}\left(\nu_{i}\right):=\nu_{i 0} x_{0}+\ldots+\nu_{i n} x_{n}$ for the homogenization of the associated linear form. Then $f_{W}\left(\nu_{0}, \ldots, \nu_{r}\right)=0$ if and only if there exists $\xi \in \bar{V}$ such that $\psi(\xi)$ lies in the linear space determined by $\nu_{0}, \ldots, \nu_{r}$. Equivalently $\xi$ lies in the linear space determined by $\psi^{*}\left(\nu_{0}\right), \ldots, \psi^{*}\left(\nu_{r}\right)$. We conclude that

$$
f_{W}=f_{V} \circ\left(\psi^{*}\right)^{r+1} \text {. }
$$

Let $v \in M_{K}^{\infty}$. Then

$$
\begin{aligned}
m\left(\sigma_{v}\left(f_{W}\right), S_{n+1}^{r+1}\right) \leq & \log \left|f_{W}\right|_{v}+(r+1) \log (n+1) \operatorname{deg} V \\
\leq & \log \left|f_{V}\right|_{v}+(r+1)\left(h_{v}(\psi)+2 \log (n+1)\right) \operatorname{deg} V+(r+1) \log (n+1) \operatorname{deg} V \\
\leq & m\left(\sigma_{v}\left(f_{V}\right)\right)+(r+1) \log (m+1) \operatorname{deg} V+(r+1)\left(h_{v}(\psi)+3 \log (n+1)\right) \operatorname{deg} V \\
\leq & m\left(\sigma\left(f_{V}\right), S_{m+1}^{r+1}\right)+\left(\sum_{i=1}^{m} 1 / 2 i\right)(r+1) \operatorname{deg} V \\
& +(r+1)\left(h_{v}(\psi)+4 \log (n+1)\right) \operatorname{deg} V .
\end{aligned}
$$

Here we have applied Lemma 1.1, Inequality (1.2) and the proof of Lemma 1.2(c), using the fact that the number of monomials of $f_{V}$ is bounded by $(n+1)^{(r+1) \operatorname{deg} V}$.

In case $v \notin M_{K}^{\infty}$ we obtain analogously $\log \left|f_{W}\right|_{v} \leq \log \left|f_{V}\right|_{v}+(r+1) h_{v}(\psi) \operatorname{deg} V$, and hence

$$
h(\psi(V)) \leq h(V)+(r+1)(h(\psi)+5 \log (n+1)) \operatorname{deg} V .
$$

Proof of Proposition 2.4.- Let $\psi: \mathbb{A}^{n} \rightarrow \mathbb{I}^{N} \times \mathbb{A}^{n}$ be the injective map $x \mapsto(\varphi(x), x)$. Then $\varphi$ decomposes as

$$
\varphi=\pi \circ \psi
$$


where $\pi: I \mathrm{~A}^{N} \times I \mathrm{~A}^{n} \rightarrow \mathbb{I}^{N}$ denotes the canonical projection. Thus

$$
\begin{aligned}
h(\varphi(V)) & \leq h(\psi(V))+3(r+1) \log (n+N+1) \operatorname{deg} \psi(V) \\
& \leq h(V)+(r+1)(h(\psi)+5 \log (n+N+1)) \operatorname{deg} V+3(r+1) \log (n+N+1) \operatorname{deg} V \\
& =h(V)+(r+1)(h(\varphi)+8 \log (n+N+1)) \operatorname{deg} V .
\end{aligned}
$$

\subsubsection{Local height of the intersection of varieties}

We obtain the following estimate for the local height of the intersection of a variety with an hypersurface. This is a consequence of our previous estimate for generalized Chow forms. This result can be seen as the local analogue of [44, Prop. 2.8], and its proof closely follows it.

Proposition 2.7 Let $V \subset \mathbb{I}^{n}$ be an equidimensional variety of dimension $r$ defined over a number field $K$. Let $f \in K\left[x_{1}, \ldots, x_{n}\right]$ be a polynomial which is not a zero-divisor in $K[V]$. We assume that both $V$ and $V \cap V(f)$ satisfy Assumption 1.5.

Then there exists $\lambda \in K^{*}$ such that

- $h_{v}(V \cap V(f)) \leq \operatorname{deg} f h_{v}(V)+h_{v}(f) \operatorname{deg} V+\log (n+1) \operatorname{deg} f \operatorname{deg} V-\log |\lambda|_{v} \quad$ for $v \in M_{K}^{\infty}$,

- $h_{v}(V \cap V(f)) \leq \operatorname{deg} f h_{v}(V)+h_{v}(f) \operatorname{deg} V-\log |\lambda|_{v} \quad$ for $v \notin M_{K}^{\infty}$.

Proof.- Set $d:=\operatorname{deg} f$ and $W:=V \cap V(f) \subset \mathbb{I A}^{n}$. By [44, Prop. 2.4] there exists $Q \in$ $K\left[U_{1}, \ldots, U_{r}\right] \backslash\{0\}$ such that $\mathcal{C} h_{d, V}(f)=Q \mathcal{C} h_{W}$. Then - as in the proof of Lemma 2.5 there exists a non-zero coefficient $\lambda$ of $Q$ such that $\log |\lambda|_{v} \leq m\left(\sigma_{v}(Q)\right)$ for all $v \in M_{K}^{\infty}$ and $\log |\lambda|_{v} \leq \log |Q|_{v}$ for all $v \notin M_{K}^{\infty}$.

Now let $v \in M_{K}^{\infty}$. From Inequality (1.2) we obtain

$$
\log |\lambda|_{v} \leq m\left(\sigma_{v}(Q)\right) \leq m\left(\sigma_{v}(Q) ; S_{n+1}^{r}\right)+r\left(\sum_{i=1}^{n} 1 / 2 i\right)(d \operatorname{deg} V-\operatorname{deg} W)
$$

since $Q$ has degree $d \operatorname{deg} V-\operatorname{deg} W$ in each group of variables. Then

$$
\begin{aligned}
h_{v}(W)= & m\left(\sigma_{v}\left(\mathcal{C} h_{W}\right) ; S_{n+1}^{r}\right)+r\left(\sum_{i=1}^{n} 1 / 2 i\right) \operatorname{deg} W \\
= & m\left(\sigma_{v}\left(\mathcal{C} h_{d, V}(f)\right) ; S_{n+1}^{r}\right)+r\left(\sum_{i=1}^{n} 1 / 2 i\right) d \operatorname{deg} V \\
& -m\left(\sigma_{v}(Q) ; S_{n+1}^{r}\right)-r\left(\sum_{i=1}^{n} 1 / 2 i\right)(d \operatorname{deg} V-\operatorname{deg} W) \\
\leq & d h_{v}(V)+h_{v}(f) \operatorname{deg} V+\log (n+1) d \operatorname{deg} V-\log |\lambda|_{v} .
\end{aligned}
$$

by straightforward application of Lemma 2.1. The case $v \notin M_{K}^{\infty}$ follows in an analogous way.

This result can be immediately generalized to families of polynomials:

Corollary 2.8 Let $V \subset \mathbb{A}^{n}$ be an equidimensional variety of dimension $r$ defined over $K$. Let $f_{1}, \ldots, f_{s} \in K\left[x_{1}, \ldots, x_{n}\right]$ be polynomials which form a complete intersection in $V$. We assume that $V \cap V\left(f_{1}, \ldots, f_{i}\right)$ satisfies Assumption 1.5 for $i=0, \ldots, s$. Set $d_{i}:=\operatorname{deg} f_{i}$.

Then there exists $\lambda \in K^{*}$ such that 
- $h_{v}\left(V \cap V\left(f_{1}, \ldots, f_{s}\right)\right) \leq \prod_{i} d_{i}\left(h_{v}(V)+\left(\sum_{i} h_{v}\left(f_{i}\right) / d_{i}\right) \operatorname{deg} V+s \log (n+1) \operatorname{deg} V\right)-\log |\lambda|_{v}$ for $v \in M_{K}^{\infty}$,

- $h_{v}\left(V \cap V\left(f_{1}, \ldots, f_{s}\right)\right) \leq \prod_{i} d_{i}\left(h_{v}(V)+\left(\sum_{i} h_{v}\left(f_{i}\right) / d_{i}\right) \operatorname{deg} V\right)-\log |\lambda|_{v} \quad$ for $v \notin M_{K}^{\infty}$.

Proof.- We just consider the case when $v$ is archimedean, as the other one follows similarly. From the preceding result we obtain

$$
\begin{aligned}
h_{v}\left(V \cap V\left(f_{1}, \ldots, f_{i}\right)\right) \leq & d_{i} h_{v}\left(V \cap V\left(f_{1}, \ldots, f_{i-1}\right)\right)+h_{v}\left(f_{i}\right) \operatorname{deg}\left(V \cap V\left(f_{1}, \ldots, f_{i-1}\right)\right) \\
& +\log (n+1) d_{i} \operatorname{deg}\left(V \cap V\left(f_{1}, \ldots, f_{i-1}\right)\right)-\log \left|\lambda_{i}\right|_{v}
\end{aligned}
$$

for some $\lambda_{i} \in K^{*}$. For the final estimate we apply iteratively this inequality and we set $\lambda:=$ $\prod_{i=1}^{s} \lambda_{i}^{d_{i+1} \cdots d_{s}}$.

Corollary 2.9 Let $f_{1}, \ldots, f_{s} \in K\left[x_{1}, \ldots, x_{n}\right]$ be polynomials which form a complete intersection in $\mathbb{I A}^{n}$. We assume that $V\left(f_{1}, \ldots, f_{i}\right)$ satisfies Assumption 1.5 for $i=1, \ldots, s$. Set $d_{i}:=\operatorname{deg} f_{i}$. Then there exists $\lambda \in K^{*}$ such that:

- $h_{v}\left(V\left(f_{1}, \ldots, f_{s}\right)\right) \leq \prod_{i} d_{i}\left(\sum_{i} h_{v}\left(f_{i}\right) / d_{i}+(n+s) \log (n+1)\right)-\log |\lambda|_{v} \quad$ for $v \in M_{K}^{\infty}$,

- $h_{v}\left(V\left(f_{1}, \ldots, f_{s}\right)\right) \leq \prod_{i} d_{i}\left(\sum_{i} h_{v}\left(f_{i}\right) / d_{i}\right)-\log |\lambda|_{v} \quad$ for $v \notin M_{K}^{\infty}$.

Proof.- We apply the previous result to $V:=\mathbb{A}^{n}$, using the fact that

$$
h_{\infty}\left(\mathbb{A}^{n}\right)=\sum_{i=1}^{n} \sum_{j=1}^{i} 1 / 2 j \leq n \log (n+1) \quad, \quad h_{p}\left(\mathbb{A}^{n}\right)=0 .
$$

The following global result is the arithmetic analogue of [21, Prop. 2.3]:

Corollary 2.10 Let $V \subset \mathbb{A}^{n}$ be a variety of dimension $r$, and let $f_{1}, \ldots, f_{s} \in \overline{\mathbb{Q}}\left[x_{1}, \ldots, x_{n}\right]$. Set $d_{i}:=\operatorname{deg} f_{i}, h:=h\left(f_{1}, \ldots, f_{s}\right)$ and $n_{0}:=\min \{r, s\}$. We assume that $d_{1} \geq \ldots \geq d_{s}$ holds. Then:

$$
h\left(V \cap V\left(f_{1}, \ldots, f_{s}\right)\right) \leq \prod_{i=1}^{n_{0}} d_{i}\left(h(V)+\left(\sum_{i=1}^{n_{0}} 1 / d_{i}\right) h \operatorname{deg} V+n_{0} \log (n+1) \operatorname{deg} V\right) .
$$

Proof.- We proceed by induction on $(r, s)$ with respect to the product order of $\mathbb{N} \times \mathbb{N}$.

The cases $r=0$ or $s=0$ are both trivial. Thus we assume $r, s \geq 1$. Let $V=\cup_{C} C$ be the decomposition of $V$ into irreducible components.

In case $C \subset V\left(f_{s}\right)$ we have that $C \cap V\left(f_{1}, \ldots, f_{s}\right)=C \cap V\left(f_{1}, \ldots, f_{s-1}\right)$ and so

$$
h\left(C \cap V\left(f_{1}, \ldots, f_{s}\right)\right) \leq \prod_{i=1}^{m_{0}} d_{i}\left(h(C)+\left(\sum_{i=1}^{m_{0}} 1 / d_{i}\right) h \operatorname{deg} C+m_{0} \log (n+1) \operatorname{deg} C\right)
$$

with $m_{0}:=\min \{r, s-1\}$.

In case $C \not \subset V\left(f_{s}\right)$ we have either $C \cap V\left(f_{s}\right)=\emptyset$ or $\operatorname{dim} C \cap V\left(f_{s}\right) \leq r-1$. The first case is trivial.

In the second case we have

$$
h\left(C \cap V\left(f_{s}\right)\right) \leq d_{s} h(C)+h \operatorname{deg} C+\log (n+1) d \operatorname{deg} C .
$$


To obtain this, we proceed as in the proof of Proposition 2.7, applying Remark 2.3 instead of Lemma 2.1.

Then we apply the inductive hypothesis and we obtain

$$
\begin{aligned}
h\left(C \cap V\left(f_{1}, \ldots, f_{s}\right)\right) \leq & \prod_{i=1}^{n_{0}-1} d_{i}\left(h\left(C \cap V\left(f_{s}\right)\right)+\left(\sum_{i=1}^{n_{0}-1} 1 / d_{i}\right) h \operatorname{deg}\left(C \cap V\left(f_{s}\right)\right)\right. \\
& \left.+\left(n_{0}-1\right) \log (n+1) \operatorname{deg}\left(C \cap V\left(f_{s}\right)\right)\right) \\
\leq & \prod_{i=1}^{n_{0}} d_{i}\left(h(C)+\left(\sum_{i=1}^{n_{0}} 1 / d_{i}\right) h \operatorname{deg} C+n_{0} \log (n+1) \operatorname{deg} C\right) .
\end{aligned}
$$

With the same notations than Corollary 2.10, for $V:=\mathbb{A}^{n}$ we obtain

$$
h\left(V\left(f_{1}, \ldots, f_{s}\right)\right) \leq \prod_{i=1}^{n_{0}} d_{i}\left(\left(\sum_{i=1}^{n_{0}} 1 / d_{i}\right) h+\left(n+n_{0}\right) \log (n+1)\right) .
$$

\subsubsection{An arithmetic Bernstein-Kushnirenko theorem}

From our estimate for the height of an affine toric variety (Proposition 1.7) and the previous results of this section we derive the following arithmetic version of the Bernstein-Kushnirenko theorem. We refer to Subsection 1.2.5 for the notation.

Proposition 2.11 Let $f_{1}, \ldots, f_{s} \in K\left[x_{1}, \ldots, x_{n}\right]$, and let $\mathcal{A} \subset\left(\mathbb{Z}_{\geq 0}\right)^{n}$ be a finite set such that $\operatorname{Supp}\left(1, x_{1}, \ldots, x_{n}, f_{1}, \ldots, f_{s}\right) \subset \mathcal{A}$. Set $d:=\max _{i} \operatorname{deg} f_{i}$ and $h:=h\left(f_{1}, \ldots, f_{s}\right)$. Then

- $\operatorname{deg} V\left(f_{1}, \ldots, f_{s}\right) \leq \operatorname{Vol}(\mathcal{A})$,

- $h\left(V\left(f_{1}, \ldots, f_{s}\right)\right) \leq\left(n h+2^{2 n+3} \log (n+1) d\right) \operatorname{Vol}(\mathcal{A})$.

Proof.- Set $\mathcal{A}:=\left\{\alpha_{1}, \ldots, \alpha_{N}\right\}$. The case $N=1$ is trivial, and so we assume $N \geq 2$. We also assume that $\alpha_{1}, \ldots, \alpha_{n}$ are the vectors of the canonical basis of $\mathbb{R}^{n}$.

The map $\varphi_{\mathcal{A}}: \mathbb{A}^{n} \rightarrow \mathbb{A}^{N}$ induces an isomorphism between $\mathbb{A}^{n}$ and the affine toric variety $X_{\mathcal{A}} \subset \mathbb{I}^{N}$. The projection map $\pi_{\mathcal{A}}: \mathbb{I}^{N} \rightarrow \mathbb{A}^{n}$ defined by $y \mapsto\left(y_{1}, \ldots, y_{n}\right)$ restricted to $X_{\mathcal{A}}$ is the inverse map of $\varphi_{\mathcal{A}}$.

For $i=1, \ldots, s$ we set $f_{i}=\sum_{j=1}^{N} a_{i j} x^{\alpha_{j}}$ and we let

$$
\ell_{i}:=\sum_{j=1}^{N} a_{i j} y_{j} \in K\left[y_{1}, \ldots, y_{N}\right]
$$

be the associated linear form. Set $V:=V\left(f_{1}, \ldots, f_{s}\right) \subset \mathbb{A}^{n}$ and $W:=X_{\mathcal{A}} \cap V\left(\ell_{1}, \ldots, \ell_{s}\right) \subset \mathbb{I}^{N}$. We have $\varphi_{\mathcal{A}}(V)=W$ and so $V=\pi_{\mathcal{A}}(W)$. Then

$$
\operatorname{deg} V \leq \operatorname{deg} W \leq \operatorname{deg} X_{\mathcal{A}}=\operatorname{Vol}(\mathcal{A})
$$

and

$$
\begin{aligned}
h(V) & \leq h(W)+3(n+1) \log (N+1) \operatorname{deg} W \\
& \leq h\left(X_{\mathcal{A}}\right)+n h \operatorname{deg}\left(X_{\mathcal{A}}\right)+4(n+1) \log (N+1) \operatorname{deg}\left(X_{\mathcal{A}}\right) \\
& \leq\left(n h+\left(2^{2 n+2} \log N+4(n+1) \log (N+1)\right) \operatorname{Vol}(\mathcal{A}) .\right.
\end{aligned}
$$

by successive application of Lemma 2.5, Corollary 2.10 and Proposition 1.7. Finally $N \leq\left(\begin{array}{c}d+n \\ n\end{array}\right)$ and so $h(V) \leq\left(n h+2^{2 n+3} \log (n+1) d\right) \operatorname{Vol}(\mathcal{A})$. 
It seems that the factor $2^{2 n}$ in the estimate of $h\left(X_{A}\right)$ is superfluous. If this is the case, the above estimate can be considerably improved. We remark that Maillot has recently obtained a much more precise estimate for the height of the isolated points of $V\left(f_{1}, \ldots, f_{s}\right)$ [39, Th. 8.48] .

\subsection{Local height of norms and traces}

Let $V \subset \mathbb{A}^{n}$ be an equidimensional variety of dimension $r$ and degree $D$ defined over a field $k$ which satisfies Assumption 1.5. As we will see below, this implies that the projection $\pi_{V}: V \rightarrow \mathbb{A}^{r}$ defined by $x \mapsto\left(x_{1}, \ldots, x_{r}\right)$ is finite (Lemma 2.13). Set $L:=k\left(\mathbb{A}^{r}\right)$ and $M:=L \otimes_{k\left[\mathbb{A}^{r}\right]} k[V]$, so that $M$ is a finite $L$-algebra of dimension $D$.

Let $f \in k\left[x_{1}, \ldots, x_{n}\right]$. We identify $\bar{f} \in k[V]$ with the multiplication map $M \rightarrow M$ defined by $q \mapsto f q$. The characteristic polynomial $\mathcal{X}_{f} \in L[t]$ of $f$ is then defined as the characteristic polynomial of this $L$-linear map. The fact that the inclusion $\pi_{V}^{*}: k\left[\mathrm{~A}^{r}\right] \hookrightarrow k[V]$ is integral implies that this polynomial lies in $k\left[\mathbb{A}^{r}\right][t]$, and we have $\mathcal{X}_{f}(\bar{f})=0$ in $k[V]$.

Set $\mathcal{X}_{f}=t^{D}+b_{D-1} t^{D-1}+\cdots+b_{0} \in k\left[\mathbb{A}^{r}\right][t]$. Then the norm $\mathrm{N}_{V}(f)$ and the trace $\operatorname{Tr}_{V}(f)$ of $f$ are defined as

$$
\mathrm{N}_{V}(f):=(-1)^{D} b_{0} \in k\left[\mathbb{I}^{r}\right] \quad, \quad \operatorname{Tr}_{V}(f):=-b_{D-1} \in k\left[\mathbb{I A}^{r}\right] .
$$

They equal the determinant and the trace of the $L$-linear map $\bar{f}: M \rightarrow M$ respectively. We also define the adjoint polynomial $f^{*}$ of $f$ as

$$
f^{*}:=(-1)^{D-1}\left(f^{D-1}+b_{D-1} f^{D-2}+\cdots+b_{1}\right) \in k\left[x_{1}, \ldots, x_{n}\right] .
$$

We have $\overline{f^{*} f}=\mathrm{N}_{V}(f)$ in $k[V]$.

The key result of this subsection is a precise bound for the height of the norm and the trace of a polynomial in case $k$ is a number field.

\subsubsection{Characteristic polynomials}

Let $V \subset \mathbb{A}^{n}$ be an equidimensional variety of dimension $r$ and degree $D$ defined over $k$. We keep notations as in Subsection 2.1.1: for $d \in \mathbb{N}$ we denote by $F:=\sum_{|\alpha| \leq d} U(d)_{0 \alpha} x^{\alpha}$ and $L_{i}:=U_{i 0}+U_{i 1} x_{1}+\cdots+U_{i n} x_{n}$ the generic polynomial of degree $d$ and 1 associated to the group of variables $U(d)_{0}$ and $U_{i}$ respectively.

As before, we set $U(d):=\left\{U(d)_{0}, U_{1}, \ldots, U_{r}\right\}$ and $N:=\left(\begin{array}{c}d+n \\ n\end{array}\right)+r(n+1)$. Also we introduce an additional group $T:=\left\{T_{0}, \ldots, T_{r}\right\}$ of $r+1$ variables which correspond to the coordinate functions of $\mathrm{IA}^{r+1}$. We consider the map

$$
\psi: \mathbb{A}^{N} \times \mathbb{A}^{n} \rightarrow \mathbb{A}^{N} \times \mathbb{A}^{r+1} \quad, \quad(U(d), x) \mapsto\left(\nu(d), F\left(\nu(d)_{0}\right)(\xi), L_{1}\left(\nu_{1}\right)(\xi), \ldots, L_{r}\left(\nu_{r}\right)(\xi)\right) .
$$

where $\nu(d):=\left(\nu(d)_{0}, \nu_{1}, \ldots, \nu_{r}\right) \in \mathbb{A}^{N}$ and $\xi \in \mathbb{A}^{n}$.

Then the Zariski closure $\overline{\psi\left(\mathbb{A}^{N} \times V\right)} \subset \mathbb{A}^{N} \times \mathbb{A}^{r+1}$ is a hypersurface, and any of its defining equations $P_{d, V} \in k[U(d)][T]$ is called a $d$-characteristic polynomial of $V$. Also we define the characteristic polynomial of $V$ by $P_{V}:=P_{1, V}$.

A $d$-characteristic polynomial is uniquely defined up to a scalar factor. In case $V$ is an irreducible variety, $P_{d, V}$ is an irreducible polynomial. When $V$ is equidimensional, it coincides with the product of $d$-characteristic polynomials of its irreducible components.

The following construction links the characteristic polynomial of a variety with its generalized Chow form. Set

$$
\zeta(d)_{0 \alpha}:= \begin{cases}U(d)_{00}-T_{0} & \text { for } \alpha=0 \\ U(d)_{0 \alpha} & \text { for } \alpha \neq 0 .\end{cases}
$$


Analogously, for $i=1, \ldots, r$ we set $\zeta_{i 0}:=U_{i 0}-T_{i}$ and $\zeta_{i j}:=U_{i j}$ for $j \neq 0$. Finally we set $\zeta(d):=\left(\zeta(d)_{0}, \zeta_{1}, \ldots, \zeta_{r}\right)$.

Lemma 2.12 Let $V \subset \mathbb{A}^{n}$ be an equidimensional variety of dimension $r$ and degree $D$. Let $f_{d, V}$ be a $d$-Chow form of $V$. Then $f_{d, V} \circ \zeta(d)$ is a $d$-characteristic polynomial of $V$.

Proof.- It is enough to consider the case when $V$ is irreducible.

Let $P_{d, V}$ be a $d$-characteristic polynomial of $V$. For $(\nu(d), \xi) \in \mathbb{A}^{N} \times V$ we set

$$
\vartheta:=\left(F\left(\nu(d)_{0}\right)(\xi), L_{1}\left(\nu_{1}\right)(\xi), \ldots, L_{r}\left(\nu_{r}\right)(\xi)\right) \in \mathbb{A}^{r+1},
$$

so that $P_{d, V}(\nu(d))(\vartheta)=0$. We observe that

$$
\left.\xi \in V \cap\left\{F\left(\nu(d)_{0}\right)(x)=\vartheta_{0}\right\} \cap\left\{L_{1}\left(\nu_{1}\right)(x)=\vartheta_{1}\right\} \cap \cdots \cap\left\{L_{r}\left(\nu_{r}\right)(x)=\vartheta_{r}\right)\right\} \subset \mathbb{A}^{n} .
$$

In particular, this variety is non-empty, and so we infer that $f_{d, V} \circ \zeta(d)(\nu(d), \vartheta)=0$. This implies that $P_{d, V} \mid f_{d, V} \circ \zeta(d)$ as $P_{d, V}$ is an irreducible polynomial.

On the other hand $f_{d, V} \circ \zeta(d)$ is also irreducible, as it is multihomogeneous and $f_{d, V} \circ \zeta(d)(U(d), 0)=$ $f_{d, V}(U(d))$. We conclude that $P_{d, V}$ and $f_{d, V} \circ \zeta(d)$ coincide up to a factor in $k^{*}$.

The previous construction shows that a $d$-characteristic polynomial of $V$ is multihomogeneous of degree $D$ in the group of variables $U(d)_{0} \cup\left\{T_{0}\right\}$ and of degree $d D$ in each group $U_{i} \cup\left\{T_{i}\right\}$.

Set $k_{d}:=\overline{k(U(d))}$, and set

$$
\phi: \mathbb{A}^{n}\left(k_{d}\right) \rightarrow \mathbb{A}^{r+1}\left(k_{d}\right) \quad, \quad x \mapsto\left(F(x), L_{1}(x), \ldots, L_{r}(x)\right) .
$$

Then $P_{d, V} \in k_{d}[T]$ is also a minimal equation for the hypersurface $\overline{\phi(V)}$, and by Bézout inequality we have also $\operatorname{deg}_{T} P_{d, V} \leq d D$ (see e.g. [47).

We assume from now on that $V$ satisfies Assumption 1.5, that is $\# \pi_{V}^{-1}(0)=\operatorname{deg} V$. In order to avoid the indeterminacy of the $d$-characteristic polynomial, we fix it as

$$
P_{d, V}:=(-1)^{D} \mathcal{C} h_{d, V} \circ \zeta(d) .
$$

In particular, we set $P_{V}:=(-1)^{D} \mathcal{C} h_{V} \circ \zeta(1)$ for the characteristic polynomial of $V$.

Set $P_{V}:=a_{D} T_{0}^{D}+\cdots+a_{0}$ for the expansion of $P_{V}$ with respect to $T_{0}$. We have that $P_{V}$ is multihomogeneous of degree $D$ in each group $U_{i} \cup\left\{T_{i}\right\}$. This implies that $a_{D}$ lies in fact in $k\left[U_{1}, \ldots, U_{r}\right]$ and is multihomogeneous of degree $D$ in each $U_{i}$ for $i=1, \ldots, r$.

Moreover, $a_{D}$ coincides with the coefficient of $U_{00}^{D}$ in $\mathcal{C} h_{V}$, and the imposed normalization on $\mathcal{C} h_{V}$ implies that

$$
a_{D}\left(e_{1}, \ldots, e_{r}\right)=\mathcal{C} h_{V}\left(e_{0}, e_{1}, \ldots, e_{r}\right)=1 .
$$

We extend the morphism $\varrho_{d}$ of Subsection 2.1.1 to a morphism $k[U(d)][T] \rightarrow k\left[U_{0}, \ldots, U_{r}\right][T]$ defining $\varrho_{d}\left(U(d)_{00}-T_{0}\right):=\left(U_{00}-T_{0}\right)^{d}$ and $\varrho_{d}\left(T_{i}\right):=T_{i}$. In other terms

$$
\varrho_{d}\left(T_{0}\right)=\sum_{j=1}^{d}(-1)^{j-1}\left(\begin{array}{l}
d \\
j
\end{array}\right) U_{00}^{d-j} T_{0}^{j} .
$$

From the previous lemma we obtain

$$
\varrho_{d}\left(P_{d, V}\right)=\varrho_{d}\left((-1)^{D} \mathcal{C} h_{d, V} \circ \zeta(d)\right)=(-1)^{D}\left(\mathcal{C} h_{V} \circ \zeta(1)\right)^{d}=(-1)^{(d+1) D} P_{V}^{d} .
$$

Now set

$$
P_{d, V}=a_{d, D} T_{0}^{D}+\cdots+a_{d, 0}
$$


for the expansion of $P_{d, V}$ with respect to $T_{0}$. The previous remark implies that $a_{d, D}=\varrho_{d}\left(a_{d, D}\right)=$ $a_{D}^{d}$. In particular $a_{d, D} \in k\left[U_{1}, \ldots, U_{r}\right]$ and $a_{d, D}\left(e_{1}, \ldots, e_{r}\right)=1$.

The following lemma allows us to obtain a characteristic polynomial of $f \in k\left[x_{1}, \ldots, x_{n}\right]$ from the $d$-characteristic polynomial of the variety $V$.

We introduce the following convention:

Given a polynomial $f \in k\left[x_{1}, \ldots, x_{n}\right]$ of degree $d$ and linear forms $\ell_{1}, \ldots, \ell_{r} \in k\left[x_{1}, \ldots, x_{n}\right]$, we denote by $P_{d, V}\left(f, \ell_{1}, \ldots, \ell_{r}\right)$ the specialization of the variables in $U(d)$ into the coefficients of $f, \ell_{1}, \ldots, \ell_{r}$.

Lemma 2.13 Let $V \subset \mathbb{A}^{n}$ be an equidimensional variety of dimension $r$ and degree $D$ which satisfies Assumption 1.8. Then the projection $\pi_{V}: V \rightarrow \mathbb{A}^{r}$ is finite.

Moreover, for a polynomial $f \in k\left[x_{1}, \ldots, x_{n}\right]$ of degree $d$, the characteristic polynomial of $f$ is given by

$$
\mathcal{X}_{f}=P_{d, V}\left(f, e_{1}, \ldots, e_{r}\right)\left(t, x_{1}, \ldots, x_{r}\right) \in k\left[\mathrm{IA}^{r}\right][t] .
$$

Proof.- We have that $P_{V}\left(U_{0}, \ldots, U_{r}\right)\left(L_{0}, \ldots, L_{r}\right)=0$ in $k[U] \otimes k[V]$ and so

$$
P_{V}\left(e_{j}, e_{1}, \ldots, e_{r}\right)\left(t, x_{1}, \ldots, x_{r}\right) \in k\left[\mathbb{A}^{r}\right][t]
$$

is a monic equation for $x_{j}$ in $k[V]$, for $j=r+1, \ldots, n$. Thus the projection $\pi_{V}$ is finite.

For the second assertion, set

$$
P_{F}(t):=P_{d, V}\left(U(d)_{0}, e_{1}, \ldots, e_{r}\right)\left(t, x_{1}, \ldots, x_{r}\right) \in k\left[U(d)_{0}\right]\left[\mathrm{A}^{r}\right][t] .
$$

This is a polynomial of degree $D$. It is monic with respect to $t$, as $a_{d, D} \in k\left[U_{1}, \ldots, U_{r}\right]$ and $a_{d, D}\left(e_{1}, \ldots, e_{r}\right)=1$. We have $P_{F}(F)=0$ in $k\left[U(d)_{0}\right] \otimes k[V]$.

Now let $m_{F}$ be the monic minimal polynomial of $F$. Let $U^{\prime}(d)_{0}$ be a group of $\left(\begin{array}{c}d+n-r \\ n-r\end{array}\right)$ variables and set $F_{0}$ for the generic polynomial of degree $d$ in the variables $x_{r+1}, \ldots, x_{n}$.

Then

$$
m_{F}\left(U^{\prime}(d)_{0}, 0\right) \in k\left[U^{\prime}(d)_{0}\right][t]
$$

is an equation for $F_{0}$ over $\pi_{V}^{-1}(0)$. Since $\pi_{V}^{-1}(0)$ is a 0 -dimensional variety of degree $D$ and $F_{0}$ separates its points, we infer that $\operatorname{deg}_{T_{0}} m_{F}=D$, and so $P_{F}=m_{F}$.

Finally we obtain

$$
\mathcal{X}_{f}=\mathcal{X}_{F}(f)=P_{F}(f)=P_{d, V}\left(f, e_{1}, \ldots, e_{r}\right)\left(t, x_{1}, \ldots, x_{r}\right)
$$

\subsubsection{Estimates for norms and traces}

Finally we prove the announced estimates for the height of the norm and the trace of a polynomial.

Lemma 2.14 Let $V \subset \mathbb{A}^{n}$ be an equidimensional variety of dimension $r$ defined over $K$ which satisfies Assumption 1.5. Let $f \in K\left[x_{1}, \ldots, x_{n}\right]$. Then

- $\operatorname{deg} \mathrm{N}_{V}(f) \leq \operatorname{deg} f \operatorname{deg} V$,

- $h_{v}\left(\mathrm{~N}_{V}(f)\right) \leq \operatorname{deg} f h_{v}(V)+h_{v}(f) \operatorname{deg} V+(r+1) \log (n+1) \operatorname{deg} f \operatorname{deg} V \quad$ for $v \in M_{K}^{\infty}$,

- $h_{v}\left(\mathrm{~N}_{V}(f)\right) \leq \operatorname{deg} f h_{v}(V)+h_{v}(f) \operatorname{deg} V \quad$ for $v \notin M_{K}^{\infty}$. 
Proof.- We keep notations as in Subsection 2.3.1. Set $d:=\operatorname{deg} f$ and $D:=\operatorname{deg} V$. We have then

$$
\mathrm{N}(f)=(-1)^{D} P_{d, V}\left(f, e_{1}, \ldots, e_{r}\right)\left(0, x_{1}, \ldots, x_{r}\right)=\mathcal{C} h_{d, V}\left(f, e_{1}-e_{0} x_{1}, \ldots, e_{r}-e_{0} x_{r}\right)
$$

by Lemmas 2.13 and 2.12. Then

$$
\operatorname{deg} \mathrm{N}(f) \leq \operatorname{deg}_{T} P_{d, V} \leq d D
$$

From the previous expression we also obtain that the coefficients of $\mathrm{N}(f)$ are some of the coefficients of $\mathcal{C} h_{d, V}(f)$, and so $|\mathrm{N}(f)|_{v} \leq\left|\mathcal{C} h_{d, V}(f)\right|_{v}$ for every absolute value $v$ of $K$.

Let $v \in M_{K}^{\infty}$. Then

$$
\begin{aligned}
\log |\mathrm{N}(f)|_{v} & \left.\leq \log \mid \mathcal{C} h_{d, V}(f)\right)\left.\right|_{v} \\
& \leq m\left(\sigma_{v}\left(\mathcal{C} h_{d, V}(f)\right) ; S_{n+1}^{r}\right)+r\left(\sum_{i=1}^{n} 1 / 2 i\right) d D+r \log (n+1) d D \\
& \leq d h_{v}(V)+h_{v}(f) D+(r+1) \log (n+1) d D
\end{aligned}
$$

by Inequalities (1.1) and (1.2), and Lemma 2.1. In a similar way we obtain $h_{v}(\mathrm{~N}(f)) \leq d h_{v}(V)+$ $h_{v}(f) D$ for $v \notin M_{K}^{\infty}$.

The proof of the following lemma follows closely that of [47, Lem. 9]. We slightly improve the degree estimate obtained therein, and we get the corresponding height estimate.

Lemma 2.15 Let $V \in \mathbb{A}^{n}$ be an equidimensional variety of dimension $r$ defined over $K$ which satisfies Assumption 1.5. Let $f, g \in K\left[x_{1}, \ldots, x_{n}\right]$ such that $\bar{f}$ is not a zero-divisor in $K[V]$. Set $d:=\max \{\operatorname{deg} f, \operatorname{deg} g\}$ and $h_{v}:=\max \left\{h_{v}(f), h_{v}(g)\right\}$ for $v \in M_{K}$. Then

- $\operatorname{deg} \operatorname{Tr}_{V}\left(f^{*} g\right) \leq d \operatorname{deg} V$,

- $h_{v}\left(\operatorname{Tr}_{V}\left(f^{*} g\right)\right) \leq d h_{v}(V)+\left(h_{v}+\log 2\right) \operatorname{deg} V+(r+1) \log (n+1) d \operatorname{deg} V \quad$ for $v \in M_{K}^{\infty}$,

- $h_{v}\left(\operatorname{Tr}_{V}\left(f^{*} g\right)\right) \leq d h_{v}(V)+h_{v} \operatorname{deg} V \quad$ for $v \notin M_{K}^{\infty}$.

Proof.- Let $D:=\operatorname{deg} V$, and let $t$ be a new variable. Then $K\left[x_{1}, \ldots, x_{r}, t\right] \hookrightarrow K\left[V \times I \mathrm{~A}^{1}\right]$ is again an integral inclusion. Set $Q(t):=\mathrm{N}_{V \times \mathbb{I}^{1}}(t f-g) \in K\left[x_{1}, \ldots, x_{r}, t\right]$. We have then $\mathrm{N}\left(t-f^{*} g\right)=\mathcal{X}_{f^{*} g}(t)$ and so

$$
\mathrm{N}_{V}(f)^{D-1} Q=\mathrm{N}_{V}\left(f^{*}\right) Q=\mathcal{X}_{f^{*} g}\left(\mathrm{~N}_{V}(f) t\right) .
$$

Set $Q=c_{D} t^{D}+\cdots+c_{0}$ with $c_{i} \in K\left[\mathrm{IA}^{r}\right]$. The last identity implies then $\operatorname{Tr}\left(f^{*} g\right)=-c_{D-1}$. Set $q>D$, and let $G_{q}$ denote the group of $q$-roots of 1 . Then $Q(\omega)=N_{V}(\omega f-g)$ for $\omega \in G_{q}$, and so

$$
\operatorname{Tr}\left(f^{*} g\right)=-\frac{1}{q} \sum_{\omega \in G_{q}} \mathrm{~N}_{V}(\omega f-g) \omega^{1-D} .
$$

From Lemma 2.14 we get $\operatorname{deg} \operatorname{Tr}\left(f^{*} g\right) \leq d D$.

For $v \in M_{K}^{\infty}$, we then obtain

$$
h_{v}\left(\operatorname{Tr}\left(f^{*} g\right)\right) \leq \max _{\omega \in G_{q}} h_{v}\left(\mathrm{~N}_{V}(\omega f-g)\right) \leq d h_{v}(V)+\left(h_{v}+\log 2\right) D+(r+1) \log (n+1) d D .
$$

Analogously, for $v \notin M_{K}^{\infty}$ we take $q>D$ such that $|q|_{v}=1$, and we obtain $h_{v}\left(\operatorname{Tr}\left(f^{*} g\right)\right) \leq$ $d h_{v}(V)+h_{v} D$. 


\section{An effective arithmetic Nullstellensatz}

In this chapter we obtain the announced estimates for the arithmetic Nullstellensatz over the ring of integers of a number field $K$. Theorem 1 of Introduction corresponds to the case $K:=\mathbb{Q}$.

These estimates depend on the number of variables and on the degree and height of the input polynomials.

\subsection{Division modulo complete intersection ideals}

The tool we will use here is Tate trace formula, which has already been used in several papers on elimination theory. One of its outstanding features is that it performs effective division modulo complete intersection ideals [17], [12], [29], [47], 115], 19]. In this section we apply trace formula to obtain sharp height estimates in the division procedure.

\subsubsection{Tate trace formula}

We describe in what follows the basic aspects of duality theory for complete intersection algebras that we will need in the sequel. We refer to Kunz [31, Appendix F] for a complete presentation of this theory.

Let $k$ be a perfect field, and set $A:=k\left[t_{1}, \ldots, t_{r}\right]$ and $A[x]:=A\left[x_{1}, \ldots, x_{n}\right]$. Let $F:=$ $\left\{F_{1}, \ldots, F_{n}\right\} \subset A[x]$ be a reduced complete intersection which defines a radical ideal $(F)$ of dimension $r$.

We consider the $A$-algebra

$$
B:=A[x] /(F)=A\left[x_{1}, \ldots, x_{n}\right] /\left(F_{1}, \ldots, F_{n}\right) .
$$

We assume that the inclusion $A \hookrightarrow B$ is finite, that is the variables $t_{1}, \ldots, t_{r}$ are in Noether normal position with respect to the variety $V:=V(F) \subset \mathbb{A}^{r+n}$. This is the case, for instance, if $V$ satisfies Assumption 1.5. Thus $B$ is a projective $A$-module, which turns to be free of rank bounded by $\operatorname{deg} V$ by Quillen-Suslin theorem.

The dual $A$-module $B^{*}:=\operatorname{Hom}_{A}(B, A)$ can be seen as a $B$-module with scalar multiplication defined by $f \cdot \tau(g):=\tau(f g)$ for $f, g \in B$ and $\tau \in B^{*}$. It is a free $B$-module of rank 1 and any of its generators is called a trace of $B$.

The following construction yields a trace $\sigma$ canonically associated to the complete intersection $F$. We take new variables $y:=\left\{y_{1}, \ldots, y_{n}\right\}$, and we set $F_{i}^{(x)}:=F_{i}(x) \in A[x]$ and $F_{i}^{(y)}:=F_{i}(y) \in$ $A[y]$. Then $F_{i}^{(y)}-F_{i}^{(x)}$ belongs to the ideal $\left(y_{1}-x_{1}, \ldots, y_{n}-x_{n}\right)$ and so there exist (not unique) $l_{i j} \in A[x, y]$ such that

$$
F_{i}^{(y)}-F_{i}^{(x)}=\sum_{j=1}^{n} l_{i j}\left(y_{j}-x_{j}\right)
$$

for $i=1, \ldots, n$. We consider the determinant $\Delta \in A[x, y]$ of the square matrix $\left(l_{i j}\right)_{i j}$, and we write it as

$$
\Delta=\sum_{m} a_{m} b_{m}
$$

with $a_{m} \in A[x]$ and $b_{m} \in A[y]$. Again, the polynomials $a_{m}, b_{m}$ are not uniquely defined. The polynomial $\Delta \in A[x, y]$ is called a pseudo-Jacobian determinant of the complete intersection $F$. Set $c_{m}:=b_{m}(x) \in A[x]$. Then there exists a unique trace $\sigma \in B^{*}$ such that for $g \in A[x]$

$$
\bar{g}=\sum_{m} \sigma\left(\bar{g} \bar{a}_{m}\right) \bar{c}_{m}
$$


where the bar denotes class modulo $(F)$.

This is what is known as Tate trace formula.

Let $J:=\operatorname{det}\left(\partial F_{i} / \partial x_{j}\right)_{i j}$ be the Jacobian determinant of the complete intersection $F$ with respect to the variables $x_{1}, \ldots, x_{n}$. Then the following identity - which justifies the name of pseudoJacobian for $\Delta$ - holds

$$
\bar{J}=\sum_{m} \bar{a}_{m} \bar{c}_{m}
$$

The standard trace $\operatorname{Tr}_{V}$ is related to $\sigma$ by the equality

$$
\operatorname{Tr}_{V}(\bar{g})=\sigma(\bar{J} \bar{g})
$$

for all $g \in A[x]$.

\subsubsection{A division lemma}

Throughout this subsection we keep notations and assumptions as in the previous one. In addition we replace $k$ by a number field $K$.

We will choose concrete polynomials $a_{m}, c_{m}$ which satisfy trace formula and we will estimate their degree and local height. Set $d:=\max _{i} \operatorname{deg} F_{i}$ and $h_{v}:=\max _{i} h_{v}\left(F_{i}\right)$ for $v \in M_{K}$.

First we choose the polynomials $l_{i j}$. Remarking that

$$
F_{i}^{(y)}-F_{i}^{(x)}=\sum_{j=1}^{n} F_{i}\left(x_{1}, \ldots, x_{j-1}, y_{j}, \ldots, y_{n}\right)-F_{i}\left(x_{1}, \ldots, x_{j}, y_{j+1}, \ldots, y_{n}\right),
$$

we set

$$
l_{i j}:=\left(F_{i}\left(x_{1}, \ldots, x_{j-1}, y_{j}, \ldots, y_{n}\right)-F_{i}\left(x_{1}, \ldots, x_{j}, y_{j+1}, \ldots, y_{n}\right)\right) /\left(y_{j}-x_{j}\right)
$$

Here we perform the division through the formula

$$
\left(y_{j}^{k}-x_{j}^{k}\right) /\left(y_{j}-x_{j}\right)=y_{j}^{k-1}+y_{j}^{k-2} x_{j}+\cdots+y_{j} x_{j}^{k-2}+x_{j}^{k-1} .
$$

We set $\Delta:=\operatorname{det}\left(l_{i j}\right)_{i j}$. Finally we choose $b_{m} \in A[y]$ as the monomials in the expansion of $\Delta$ with respect to $y, a_{m} \in A[x]$ as the corresponding coefficient, and we set $c_{m}:=b_{m}(x)$.

Set $F_{i}=\sum_{\alpha} a_{i \alpha} x^{\alpha}$ with $a_{i \alpha} \in A$. Then

$$
l_{i j}=\sum_{\alpha} a_{i \alpha} x_{1}^{\alpha_{1}} \cdots x_{j-1}^{\alpha_{j-1}} y_{j+1}^{\alpha_{j+1}} \cdots y_{n}^{\alpha_{n}}\left(y_{j}^{\alpha_{j}-1}+\cdots+x_{j}^{\alpha_{j}-1}\right) \in A[x, y] .
$$

We deduce that $\operatorname{deg} l_{i j} \leq d-1$ and $h_{v}\left(l_{i j}\right) \leq h_{v}$ for every $v \in M_{K}$. Then $\operatorname{deg} \Delta \leq n(d-1)$ and so

$$
\operatorname{deg} a_{m}+\operatorname{deg} c_{m} \leq n(d-1) .
$$

We have also $h_{v}\left(c_{m}\right)=0$ and $h_{v}\left(a_{m}\right) \leq h_{v}(\Delta)$.

Finally we can write

$$
l_{i j}=C_{0}+\cdots+C_{d-1} y_{j}^{d-1}
$$

where each $C_{k} \in A\left[x_{1}, \ldots, x_{j}, y_{j+1}, \ldots, y_{n}\right]$ is a polynomial in $n+r$ variables of degree bounded by $d-1$. This implies that the number of monomials of $l_{i j}$ is bounded by $d\left(\begin{array}{c}n+r+d-1 \\ n+r\end{array}\right) \leq d(n+$ $r+1)^{d-1}$.

Therefore, for $v \in M_{K}^{\infty}$ we have

$$
\begin{aligned}
h_{v}\left(a_{m}\right) & \leq h_{v}(\Delta) \\
& \leq n h_{v}+(n-1)(\log d+(d-1) \log (n+r+1))+n \log n \\
& \leq n\left(h_{v}+d \log (n+r+1)+\log d\right) .
\end{aligned}
$$


Analogously we have $h_{v}\left(a_{m}\right) \leq n h_{v}$ for $v \notin M_{K}^{\infty}$.

The following is a sharp estimate for the degree and the local height of the polynomials in the division procedure. It is a substantial improvement over [29, Thm. 29].

We introduce the notation $\operatorname{deg}_{t} f$ and $\operatorname{deg}_{x} f$ for the degree of a polynomial $f \in A[x]$ with respect to the group of variables $t$ and $x$, respectively.

Main Lemma 3.1 (Division Lemma)

Set $A:=K\left[t_{1}, \ldots, t_{r}\right]$ and $A[x]:=A\left[x_{1}, \ldots, x_{n}\right]$. Let $F:=\left\{F_{1}, \ldots, F_{n}\right\} \subset A[x]$ be a reduced complete intersection defining a variety $V:=V(F) \subset \mathbb{A}^{r+n}$ which satisfies Assumption 1.5. Set $B:=K[V]=A[x] /(F)$.

Let $f, g \in A[x]$ be polynomials such that $\bar{f} \in B$ is a non-zero divisor and $\bar{f} \mid \bar{g}$ in $B$. Set $d:=\max \left\{\operatorname{deg} f, \operatorname{deg} F_{1}, \ldots, \operatorname{deg} F_{n}\right\}$ and $h_{v}:=\max \left\{h_{v}(f), h_{v}\left(F_{1}\right), \ldots, h_{v}\left(F_{n}\right)\right\}$ for $v \in M_{K}$.

Then there exist $q \in A[x]$ and $\xi \in K^{*}$ such that

- $\bar{q} \bar{f}=\bar{g}$

- $\operatorname{deg}_{x} q \leq n d$,

- $\operatorname{deg} q \leq \operatorname{deg}_{t} g+\left(n d+\max \left\{(n+1) d, \operatorname{deg}_{x} g\right\}\right) \operatorname{deg} V$,

- $h_{v}(q) \leq h_{v}(g)+\left(n d+\max \left\{d, \operatorname{deg}_{x} g\right\}\right) h_{v}(V)$

$+\left((n+1) h_{v}+(r+6) \log (n+r+1)\left(n d+\max \left\{(n+1) d, \operatorname{deg}_{x} g\right\}\right)\right) \operatorname{deg} V$

$+2 \log (r+1) \operatorname{deg}_{t} g-\log |\xi|_{v}$

for $v \in M_{K}^{\infty}$,

- $h_{v}(q) \leq h_{v}(g)+\left(n d+\max \left\{d, \operatorname{deg}_{x} g\right\}\right) h_{v}(V)+(n+1) h_{v} \operatorname{deg} V-\log |\xi|_{v}$

for $v \notin M_{K}^{\infty}$.

Proof.- Set $L:=K\left(t_{1}, \ldots, t_{r}\right)$ for the quotient field of $A$ and $M:=L \otimes_{A} B$. Then $M$ is a finite $L$-algebra of dimension $\operatorname{deg} V$ and $\sigma$ can be uniquely extended to a $L$-linear map $\sigma: M \rightarrow M$.

The fact that $B$ is a torsion-free $A$-algebra implies that the canonical map $B \rightarrow M$ is an inclusion.

We will only consider the case $n \geq 1$. For the case $n=0$ we refer to Remark 3.2. Whenever it is clear from the context, we will avoid explicit reference to the ring in which we are considering a given element of $A[x]$.

Let $q_{0} \in A[x]$ be any polynomial such that $q_{0} f=g$ in $B$. We have that $f$ is a non-zero divisor in $B$, and so it is invertible in $M$. Then $q_{0}=f^{-1} g$ in $M$ and therefore $\sigma\left(f^{-1} g p\right)=\sigma\left(q_{0} p\right) \in A$ for all $p \in A[x]$. Then we set

$$
q:=\sum_{m} \sigma\left(f^{-1} g a_{m}\right) c_{m} \in A[x] .
$$

Tate trace formula implies that $q \equiv q_{0} \quad(\bmod (F))$, and so $q f=g$ in $B$.

Let $J \in A[x]$ denote the Jacobian determinant of the complete intersection $F$ with respect to the group of variables $x$. This is a non-zero divisor because of the Jacobian criterion, and so it is also invertible in $M$.

Let $(J f)^{*}$ be the adjoint polynomial of $J f$ and set

$$
\Lambda_{m}:=\operatorname{Tr}_{V}\left((J f)^{*} g a_{m}\right) \in A .
$$

We have $J f(J f)^{*}=\mathrm{N}(J f) \in A \backslash\{0\}$, and so

$$
\Lambda_{m} / \mathrm{N}(J f)=\operatorname{Tr}\left((J f)^{-1} g a_{m}\right)=\sigma\left(f^{-1} g a_{m}\right) \in A .
$$


In particular $\mathrm{N}(J f) \mid \Lambda_{m}$ in $A$, and we have the expression

$$
q=\frac{1}{\mathrm{~N}(J f)} \sum_{m} \Lambda_{m} c_{m}
$$

Clearly

$$
\operatorname{deg}_{x} q \leq \max _{m} \operatorname{deg} c_{m} \leq n(d-1) \leq n d .
$$

Next we analyze the total degree of $q$. Let $g:=\sum_{\alpha} p_{\alpha} x^{\alpha}$ be the monomial expansion of $g$ with respect to $x$. Then

$$
\Lambda_{m}=\sum_{\alpha} p_{\alpha} \operatorname{Tr}\left((J f)^{*} x^{\alpha} a_{m}\right),
$$

as $\operatorname{Tr}$ is a $A$-linear map. We have the estimates $\operatorname{deg}(J f) \leq n(d-1)+d \leq(n+1) d$ and $\operatorname{deg}\left(x^{\alpha} a_{m}\right) \leq \operatorname{deg}_{x} g+\operatorname{deg} a_{m}$, from where we get

$$
\operatorname{deg} \operatorname{Tr}\left((J f)^{*} x^{\alpha} a_{m}\right) \leq \max \left\{(n+1) d, \operatorname{deg}_{x} g+\operatorname{deg} a_{m}\right\} \operatorname{deg} V
$$

by Lemma 2.15. Thus

$$
\begin{aligned}
\operatorname{deg} q & \leq \operatorname{deg}_{t} g+\max _{m}\left\{\max \left\{(n+1) d, \operatorname{deg}_{x} g+\operatorname{deg} a_{m}\right\} \operatorname{deg} V+\operatorname{deg} c_{m}\right\} \\
& \leq \operatorname{deg}_{t} g+\max _{m}\left\{\max \left\{(n+1) d+\operatorname{deg} c_{m}, \operatorname{deg}_{x} g+\operatorname{deg} a_{m}+\operatorname{deg} c_{m}\right\}\right\} \operatorname{deg} V \\
& \leq \operatorname{deg}_{t} g+\max \left\{(n+1) d+n d, \operatorname{deg}_{x} g+n d\right\} \operatorname{deg} V \\
& \leq \operatorname{deg}_{t} g+\left(n d+\max \left\{(n+1) d, \operatorname{deg}_{x} g\right\}\right) \operatorname{deg} V .
\end{aligned}
$$

For the rest of the proof, we will use several times the following basic estimates:

$$
\begin{aligned}
\max \left\{\operatorname{deg}(J f), \operatorname{deg}\left(x^{\alpha} a_{m}\right)\right\} & \leq n d+\max \left\{d, \operatorname{deg}_{x} g\right\}, \\
\operatorname{deg} \operatorname{Tr}\left((J f)^{*} x^{\alpha} a_{m}\right) & \leq\left(n d+\max \left\{d, \operatorname{deg}_{x} g\right\}\right) \operatorname{deg} V .
\end{aligned}
$$

Finally we estimate the local height of $q$. Let $v \in M_{K}^{\infty}$. We have $h_{v}\left(\partial F_{i} / \partial x_{j}\right) \leq h_{v}+\log d$ and so

$h_{v}(J) \leq n\left(h_{v}+\log d\right)+(n-1) \log (n+r+1)(d-1)+n \log n \leq n\left(h_{v}+\log (n+r+1) d+\log d\right)$.

Therefore

$$
\begin{aligned}
h_{v}(J f) & \leq n\left(h_{v}+\log (n+r+1) d+\log d\right)+h_{v}+\log (n+r+1) d \\
& \leq(n+1) h_{v}+(n+1) \log (n+r+1) d+n \log d
\end{aligned}
$$

by Lemma 1.2(b). We recall that $h_{v}\left(x^{\alpha} a_{m}\right) \leq n\left(h_{v}+\log (n+r+1) d+\log d\right)$ by Inequality (3.1) and so

$$
\max \left\{h_{v}(J f), h_{v}\left(x^{\alpha} a_{m}\right)\right\} \leq(n+1) h_{v}+(n+1) \log (n+r+1) d+n \log d .
$$

Then

$$
\begin{aligned}
h_{v}\left(\operatorname{Tr}\left((J f)^{*} x^{\alpha} a_{m}\right)\right) \leq & \left(n d+\max \left\{d, \operatorname{deg}_{x} g\right\}\right) h_{v}(V) \\
& +\left((n+1) h_{v}+(n+1) \log (n+r+1) d+n \log d+\log 2\right) \operatorname{deg} V \\
& +(r+1) \log (n+r+1)\left(n d+\max \left\{d, \operatorname{deg}_{x} g\right\}\right) \operatorname{deg} V \\
\leq & \left(n d+\max \left\{d, \operatorname{deg}_{x} g\right\}\right) h_{v}(V)+\left((n+1) h_{v}+((2 n+1) \log (n+r+1) d) \operatorname{deg} V\right. \\
& +(r+1) \log (n+r+1)\left(n d+\max \left\{d, \operatorname{deg}_{x} g\right\}\right) \operatorname{deg} V \\
\leq & \left(n d+\max \left\{d, \operatorname{deg}_{x} g\right\}\right) h_{v}(V) \\
& +\left((n+1) h_{v}+(r+2) \log (n+r+1)\left(n d+\max \left\{(n+1) d, \operatorname{deg}_{x} g\right\}\right)\right) \operatorname{deg} V
\end{aligned}
$$


by Lemma 2.15. Hence

$$
\begin{aligned}
h_{v}\left(\Lambda_{m}\right) \leq & \max _{\alpha}\left\{h_{v}\left(p_{\alpha} \operatorname{Tr}\left((J f)^{*} x^{\alpha} a_{m}\right)\right\}+\log (n+1) \operatorname{deg}_{x} g\right. \\
\leq & h_{v}(g)+\max _{\alpha}\left\{h_{v}\left(\operatorname{Tr}\left((J f)^{*} x^{\alpha} a_{m}\right)\right)\right\} \\
& +\log (r+1)\left(n d+\max \left\{d, \operatorname{deg}_{x} g\right\}\right) \operatorname{deg} V+\log (n+1) \operatorname{deg}_{x} g \\
\leq & h_{v}(g)+\left(n d+\max \left\{d, \operatorname{deg}_{x} g\right\}\right) h_{v}(V) \\
& +\left((n+1) h_{v}+(r+2) \log (n+r+1)\left(n d+\max \left\{(n+1) d, \operatorname{deg}_{x} g\right\}\right)\right) \operatorname{deg} V \\
& +\log (r+1)\left(n d+\max \left\{d, \operatorname{deg}_{x} g\right\}\right) \operatorname{deg} V+\log (n+1) \operatorname{deg}_{x} g \\
\leq & h_{v}(g)+\left(n d+\max \left\{d, \operatorname{deg}_{x} g\right\}\right) h_{v}(V) \\
& +\left((n+1) h_{v}+(r+4) \log (n+r+1)\left(n d+\max \left\{(n+1) d, \operatorname{deg}_{x} g\right\}\right)\right) \operatorname{deg} V
\end{aligned}
$$

by application of Identity (3.2) and Lemma 1.2(b). We have

$$
h_{v}(q) \leq \max _{m}\left\{h_{v}\left(\Lambda_{m} / \mathrm{N}(J f)\right)\right\}
$$

as each $c_{m}$ is a different monomial in $x$. Thus it only remains to estimate the local height of each $\Lambda_{m} / N(J f)$. Let $\xi \in K^{*}$ be any non-zero coefficient of $\mathrm{N}(J f)$. Then

$$
\begin{aligned}
\log \left|\Lambda_{m} / \mathrm{N}(J f)\right|_{v} \leq & h_{v}\left(\Lambda_{m}\right)+2 \log (r+1)\left(\operatorname{deg}_{t} g+\left(n d+\max \left\{d, \operatorname{deg}_{x} g\right\}\right) \operatorname{deg} V\right)-\log |\mathrm{N}(J f)|_{v} \\
\leq & h_{v}(g)+\left(n d+\max \left\{d, \operatorname{deg}_{x} g\right\}\right) h_{v}(V) \\
& +\left((n+1) h_{v}+(r+6) \log (n+r+1)\left(n d+\max \left\{(n+1) d, \operatorname{deg}_{x} g\right\}\right)\right) \operatorname{deg} V \\
& +2 \log (r+1) \operatorname{deg}_{t} g-\log |\xi|_{v}
\end{aligned}
$$

by Lemma 1.2(d) and the fact that $\log |\xi|_{v} \leq \log |\mathrm{N}(J f)|_{v}$. From Lemma 2.14 and Inequality (3.3) we obtain

$$
\begin{aligned}
\log |\xi|_{v} \leq & h_{v}(\mathrm{~N}(J f)) \\
\leq & (n+1) d h_{v}(V)+\left((n+1) h_{v}+(n+1) \log (n+r+1) d+n \log d\right) \operatorname{deg} V \\
& +(r+1)(n+1) \log (n+r+1) d \operatorname{deg} V \\
\leq & (n+1) d h_{v}(V)+\left((n+1) h_{v}+(r+3)(n+1) \log (n+r+1) d\right) \operatorname{deg} V
\end{aligned}
$$

This implies that the right hand side of Inequality (3.4) is non-negative. So the inequality also holds for $h_{v}\left(\Lambda_{m} / \mathrm{N}(J f)\right)$, and thus for $h_{v}(q)$.

The case $v \notin M_{K}^{\infty}$ is treated analogously. We remark that the election of $\xi$ is independent of $v$, and so it can be done uniformly.

Remark 3.2 Let notations be as in the previous lemma. In case $n=0$ we have the sharper estimates

- $\operatorname{deg} q \leq \operatorname{deg} g$,

- $h_{v}(q) \leq h_{v}(g)+h_{v}+2 \log (r+1) \operatorname{deg} g-\log |\xi|_{v} \quad$ for $v \in M_{K}^{\infty}$,

- $h_{v}(q) \leq h_{v}(g)+h_{v}-\log |\xi|_{v} \quad$ for $v \notin M_{K}^{\infty}$.

Here $\xi \in K^{*}$ denotes any non-zero coefficient of $f$. The local height estimates follow from Lemma $1.9(d)$ and the fact that $h_{v}-\log |\xi|_{v} \geq 0$. 


\subsection{An effective arithmetic Nullstellensatz}

\subsubsection{Estimates for the complete intersection case}

The following result gives estimates for the degree and local height of polynomials arising in the Nullstellensatz over a number field $K$ in case the input is a reduced weak regular sequence, i.e. when the input is a reduced regular sequence which eventually may have no common zeros in $I^{n}$. It is a direct consequence of the division lemma above.

These estimates depend mainly on the degree and height of the varieties successively cut out by the input polynomials. They are quite flexible apply to other situations as we will see in Chapter 4 .

Lemma 3.3 Let $n \geq 2$ and let $f_{1}, \ldots, f_{s} \in K\left[x_{1}, \ldots, x_{n}\right]$ be polynomials without common zeros in $I^{n}$ which form a reduced weak regular sequence. Furthermore assume that for $j=1, \ldots, s-1$, $V_{j}:=V\left(f_{1}, \ldots, f_{j}\right)$ satisfies Assumption 1.5 .

Set $d:=\max _{i}\left\{\operatorname{deg} f_{i}, 2\right\}$ and $h_{v}:=\max _{i} h_{v}\left(f_{i}\right)$ for $v \in M_{K}$.

Then there exist $p_{1}, \ldots, p_{s} \in K\left[x_{1}, \ldots, x_{n}\right]$ and $\xi \in K^{*}$ such that

- $1=p_{1} f_{1}+\cdots+p_{s} f_{s}$,

- $\operatorname{deg} p_{i} \leq 2 n d\left(1+\sum_{j=1}^{\min \{n, s\}-1} \operatorname{deg} V_{j}\right)$,

- $h_{v}\left(p_{i}\right) \leq 2 n d \sum_{j=1}^{s-1} h_{v}\left(V_{j}\right)+\left((n+1) h_{v}+2 n(2 n+5) \log (n+1) d\right)\left(1+\sum_{j=1}^{s-1} \operatorname{deg} V_{j}\right)-\log |\xi|_{v}$ for $v \in M_{K}^{\infty}$,

- $h_{v}\left(p_{i}\right) \leq 2 n d \sum_{j=1}^{s-1} h_{v}\left(V_{j}\right)+(n+1) h_{v}\left(1+\sum_{j=1}^{s-1} \operatorname{deg} V_{j}\right)-\log |\xi|_{v} \quad$ for $v \notin M_{K}^{\infty}$.

Proof.- Set $I_{i}:=I\left(V_{i}\right)=\left(f_{1}, \ldots, f_{i}\right)$ for $i=0, \ldots, s-1$. Also set $A_{i}:=K\left[x_{1}, \ldots, x_{n-i}\right]$ and $B_{i}:=K\left[V_{i}\right]=K\left[x_{1}, \ldots, x_{n}\right] / I_{i}$. The fact that $V_{i}$ satisfies Assumption 1.5 implies that the inclusion $A_{i} \hookrightarrow B_{i}$ is integral.

We note that the sets of free and dependent variables of $B_{i}$ have cardinality $n-i$ and $i$ respectively. Also the set of dependent variables of $B_{j}$ is contained in that of $B_{i}$ for $i \leq j$.

For $f \in K\left[x_{1}, \ldots, x_{n}\right]$ we denote by $\operatorname{deg}_{x(i)} f$ the degree of $f$ in the dependent variables $x_{n-i+1}, \ldots, x_{n}$ of $B_{i}$ with respect to the integral inclusion $A_{i} \hookrightarrow B_{i}$. For $i \leq j$, the previous observation implies that $\operatorname{deg}_{x(j)} f \leq \operatorname{deg}_{x(i)} f$.

Applying the Division Lemma 3.1, we will construct inductively polynomials $p_{1}, \ldots, p_{s}$ : first we take $p_{s}$ such that

$$
p_{s} f_{s} \equiv 1 \quad\left(\bmod I_{s-1}\right) .
$$

For $0 \leq i \leq s-2$ we assume that $p_{i+2}, \ldots, p_{s}$ are already constructed and we set

$$
b_{i+1}:=1-\left(p_{i+2} f_{i+2}+\cdots+p_{s} f_{s}\right) .
$$

Then $f_{i+1}$ is a non-zero divisor and $f_{i+1} \mid b_{i+1}$ in $B_{i}$. We apply again Division Lemma to obtain $p_{i+1}$ such that

$$
p_{i+1} f_{i+1} \equiv b_{i+1} \quad\left(\bmod I_{i}\right),
$$

Continuing this procedure until $i=0$, we get $1=p_{1} f_{1}+\cdots+p_{s} f_{s}$ in $K\left[x_{1}, \ldots, x_{n}\right]$.

Let us analyze degrees.

First we consider the case $s \leq n$. Again we proceed by induction.

First we have $\operatorname{deg}_{x(s-1)} p_{s} \leq(s-1) d \leq(n-1) d$ and $\operatorname{deg} p_{s} \leq(2 s-1) d \operatorname{deg} V_{s-1}$. 
Now let $1 \leq i \leq s-2$. Then $\operatorname{deg}_{x(i)} p_{i+1} \leq i d$ and

$$
\operatorname{deg} p_{i+1} \leq \operatorname{deg} b_{i+1}+\left(i d+\max \left\{(i+1) d, \operatorname{deg}_{x(i)} b_{i+1}\right\}\right) \operatorname{deg} V_{i} .
$$

where

$$
\operatorname{deg}_{x(i)} b_{i+1} \leq \max _{j \geq i+2}\left\{\operatorname{deg}_{x(i)} p_{j}+\operatorname{deg} f_{j}\right\} \leq \max _{j \geq i+2} \operatorname{deg}_{x(j-1)} p_{j}+d \leq s d
$$

Hence

$$
\begin{aligned}
\operatorname{deg} p_{i+1} & \leq \max _{j \geq i+2} \operatorname{deg} p_{j}+d+(s+i) d \operatorname{deg} V_{i} \\
& \leq(2 s-1) d \operatorname{deg} V_{s-1}+\sum_{j=i}^{s-2}\left(d+(s+j) d \operatorname{deg} V_{j}\right) \\
& =(s-i-1) d+\sum_{j=i}^{s-1}(s+j) d \operatorname{deg} V_{j} .
\end{aligned}
$$

For $i=0$ we have $p_{1} \mid b_{1}$ and therefore $\operatorname{deg} p_{1} \leq \operatorname{deg} b_{1} \leq \max _{j \geq 2} \operatorname{deg} p_{j}+d$. Then for all $i$ :

$$
\operatorname{deg} p_{i} \leq(s-1) d+\sum_{j=1}^{s-1}(s+j) d \operatorname{deg} V_{j} \leq 2 n d\left(1+\sum_{j=1}^{s-1} \operatorname{deg} V_{j}\right)
$$

Next we consider the case $s=n+1$. In this case $V_{s}$ is a 0 -dimensional variety and so

$$
\operatorname{deg} p_{n+1}=\operatorname{deg}_{x(n)} p_{n+1} \leq n d .
$$

Let $1 \leq i \leq n-1$. Then $\operatorname{deg}_{x(i)} p_{i+1} \leq i d$ and

$$
\begin{aligned}
\operatorname{deg} p_{i+1} & \leq \max _{j \geq i+2} \operatorname{deg} p_{j}+d+(n+1+i) d \operatorname{deg} V_{i} \\
& \leq n d+\sum_{j=i}^{n-1}\left(d+(n+1+j) d \operatorname{deg} V_{j}\right) \\
& =(2 n-i) d+\sum_{j=i}^{n-1}(n+1+j) d \operatorname{deg} V_{j} .
\end{aligned}
$$

We have also $\operatorname{deg} p_{1} \leq \operatorname{deg} b_{1} \leq \max \operatorname{deg}_{j \geq 2} \operatorname{deg} p_{j}+d$. We conclude for all $i$ :

$$
\operatorname{deg} p_{i} \leq 2 n d+\sum_{j=1}^{n-1}(n+1+j) d \operatorname{deg} V_{j} \leq 2 n d\left(1+\sum_{j=1}^{n-1} \operatorname{deg} V_{j}\right)
$$

Finally we estimate the local height of these polynomials. In the rest of the proof we will make repeated use of the following degree bounds:

$$
\begin{aligned}
\operatorname{deg}_{x(i-1)} p_{i} & \leq n d, \\
\operatorname{deg} p_{i} & \leq 2 n d\left(1+\sum_{j=i-1}^{\min \{n, s\}-1} \operatorname{deg} V_{j}\right) .
\end{aligned}
$$

As usual, we consider only the case $v \in M_{K}^{\infty}$, the case $v \notin M_{K}^{\infty}$ can be treated analogously. From Division Lemma we obtain

$h_{v}\left(p_{s}\right) \leq s d h_{v}\left(V_{s-1}\right)+\left(s h_{v}+(n-(s-1)+6)(s+(s-1)) \log (n+1) d\right) \operatorname{deg} V_{s-1}-\log \left|\xi_{s-1}\right|_{v}$ 
for some $\xi_{s-1} \in K^{*}$.

Let $1 \leq i \leq s-2$ and set $n_{0}:=\min \{n, s\}$. Then there exists $\xi_{i} \in K^{*}$ such that

$$
\begin{aligned}
h_{v}\left(p_{i+1}\right) \leq & h_{v}\left(b_{i+1}\right)+\left(i d+\max \left\{d, \operatorname{deg}_{x(i)} b_{i+1}\right\}\right) h_{v}\left(V_{i}\right) \\
& +\left((i+1) h_{v}+(n-i+6) \log (n+1)\left(i d+\max \left\{(i+1) d, \operatorname{deg}_{x(i)} b_{i+1}\right\}\right)\right) \operatorname{deg} V_{i} \\
& +2 \log (n-i+1) \operatorname{deg} b_{i+1}-\log \left|\xi_{i}\right|_{v} \\
\leq & \max _{j \geq i+2} h_{v}\left(p_{j}\right)+h_{v}+\log (n+1) d+\log (s-i)+(s+i) d h_{v}\left(V_{i}\right)+(i+1) h_{v} \operatorname{deg} V_{i} \\
& +(n-i+6)(s+i) \log (n+1) d \operatorname{deg} V_{i}+2 \log (n+1)\left(2 n d\left(1+\sum_{j=i+1}^{n_{0}-1} \operatorname{deg} V_{j}\right)+d\right) \\
& -\log \left|\xi_{i}\right|_{v} .
\end{aligned}
$$

Applying the inductive hypothesis we obtain

$$
\begin{aligned}
h_{v}\left(p_{i+1}\right) \leq & s d h_{v}\left(V_{s-1}\right)+d \sum_{j=i}^{s-2}(s+j) h_{v}\left(V_{j}\right)+(s-i-1) h_{v}+h_{v} \sum_{j=i}^{s-1}(j+1) \operatorname{deg} V_{j} \\
& +4(s-i-1)(n+1) \log (n+1) d+\log (n+1) d \sum_{j=i}^{s-1}(n-j+6)(s+j) \operatorname{deg} V_{j} \\
& +4 n \log (n+1) d \sum_{j=i+1}^{n_{0}-1}(j-i) \operatorname{deg} V_{j}-\sum_{j=i}^{s-1} \log \left|\xi_{j}\right|_{v} .
\end{aligned}
$$

For $i=0$ we apply Remark 3.2: there exists $\xi_{0} \in K^{*}$ such that

$$
\begin{aligned}
h_{v}\left(p_{1}\right) \leq & h_{v}\left(b_{1}\right)+h_{v}+2 \log (n+1) \operatorname{deg} b_{1}-\log \left|\xi_{0}\right|_{v} \\
\leq & \max _{j \geq 2} h_{v}\left(p_{j}\right)+2 h_{v}+\log (n+1) d+\log s+2 \log (n+1)\left(2 n d\left(1+\sum_{j=1}^{n_{0}-1} \operatorname{deg} V_{j}\right)+d\right) \\
& -\log \left|\xi_{0}\right|_{v} .
\end{aligned}
$$

We set $\xi:=\prod_{j=0}^{s-1} \xi_{j}$. Then

$$
\begin{aligned}
h_{v}\left(p_{1}\right) \leq & 2 n d \sum_{j=1}^{s-1} h_{v}\left(V_{j}\right)+(n+1) h_{v}\left(1+\sum_{j=1}^{s-1} \operatorname{deg} V_{j}\right)+4 n(n+1) \log (n+1) d \\
& +\log (n+1) d \sum_{j=1}^{s-1}(n-j+6)(n+1+j) \operatorname{deg} V_{j}+4 n \log (n+1) d \sum_{j=1}^{n_{0}-1} j \operatorname{deg} V_{j}-\log |\xi|_{v} \\
\leq & 2 n d \sum_{j=1}^{s-1} h\left(V_{j}\right)+\left((n+1) h_{v}+2 n(2 n+5) \log (n+1) d\right)\left(1+\sum_{j=1}^{s-1} \operatorname{deg} V_{j}\right)-\log |\xi|_{v} .
\end{aligned}
$$

This last inequality follows from the facts that $4 n j+(n-j+6)(j+s) \leq 2 n(2 n+5)$ for $j \leq n-1$, and $6(2 n+1) \leq 2 n(2 n+5)$ as $n \geq 2$.

To conclude the proof, observe that for $i=1, \operatorname{dots}, s-1$, Inequality 3.5 guarantees that the obtained estimate for $p_{i}$ differs from the one for $p_{i+1}$ by a positive term. Thus, the same estimate holds for $h_{v}\left(p_{i}\right), 1 \leq i \leq s$.

By means of Bézout inequality, we can now estimate the degree and height of the varieties $V_{j}$. In this way we obtain an estimate which only depends on the degree and height of the input polynomials. 
Corollary 3.4 Let notations and assumptions be as in Lemma 3.3. Then there exist $p_{1}, \ldots, p_{s} \in$ $K\left[x_{1}, \ldots, x_{n}\right]$ and $\gamma \in K^{*}$ such that

- $1=p_{1} f_{1}+\cdots+p_{s} f_{s}$,

- $\operatorname{deg} p_{i} \leq 4 n d^{n}$,

- $h_{v}\left(p_{i}\right) \leq 4 n(n+1) d^{n} h_{v}+4 n(4 n+5) \log (n+1) d^{n+1}-\log |\gamma|_{v} \quad$ for $v \in M_{K}^{\infty}$,

- $h_{v}\left(p_{i}\right) \leq 4 n(n+1) d^{n} h_{v}-\log |\gamma|_{v} \quad$ for $v \notin M_{K}^{\infty}$.

Proof.- Let us first consider degrees. We assume without loss of generality $d \geq 2$. From the preceding result we obtain

$$
\operatorname{deg}\left(p_{i}\right) \leq 2 n d\left(1+\sum_{j=1}^{\min \{n, s\}-1} \operatorname{deg} V_{j}\right) \leq 2 n d\left(1+\cdots+d^{n-1}\right) \leq 4 n d^{n} .
$$

Next we consider the local height estimates. Let $v \in M_{K}^{\infty}$. We have

$$
h_{v}\left(p_{i}\right) \leq 2 n d \sum_{j=1}^{s-1} h_{v}\left(V_{j}\right)+\left((n+1) h_{v}+2 n(2 n+5) \log (n+1) d\right)\left(1+\sum_{j=1}^{s-1} \operatorname{deg} V_{j}\right)-\log |\xi|_{v}
$$

for some $\xi \in K^{*}$. Applying Corollary 2.9, $\quad h_{v}\left(V_{j}\right) \leq j d^{j-1} h_{v}+(n+j) \log (n+1) d^{j}-\log \left|\lambda_{j}\right|_{v}$ for some $\lambda_{j} \in K^{*}$. Therefore

$$
\begin{aligned}
h_{v}\left(p_{i}\right) \leq & 2 n d \sum_{j=1}^{s-1}\left(j d^{j-1} h_{v}+(n+j) \log (n+1) d^{j}-\log \left|\lambda_{j}\right|_{v}\right) \\
& +\left((n+1) h_{v}+2 n(2 n+5) \log (n+1) d\right) \sum_{j=0}^{n} d^{i}-\log |\xi|_{v} \\
\leq & 4 n^{2} d^{n} h_{v}+8 n^{2} \log (n+1) d^{n+1} \\
& +2(n+1) d^{n} h_{v}+4 n(2 n+5) \log (n+1) d^{n+1}-2 n d \sum_{j=1}^{s-1} \log \left|\lambda_{j}\right|_{v}-\log |\xi|_{v} \\
\leq & 4 n(n+1) d^{n} h_{v}+4 n(4 n+5) \log (n+1) d^{n+1}-\log |\gamma|_{v},
\end{aligned}
$$

where $\gamma \in K^{*}$ is defined as $\gamma:=\xi \prod_{j=1}^{s-1} \lambda_{j}^{2 n d}$.

The case $v \notin M_{K}^{\infty}$ follows anagolously.

\subsubsection{Proof of Theorem 1}

In order to prove Theorem 1, it only remains to put the case of a general input into the hypothesis of Corollary 3.4. This is accomplished by replacing the input polynomials and variables by generic linear combinations. The coefficients of the linear combinations will be chosen to be roots of 1 . Amazingly enough, we will see using next lemma that we don't need to control the degree of the involved number field extension.

Let $L$ be a finite extension of $K$, and let $\mathcal{B}:=\left\{e_{1}, \ldots, e_{N}\right\}$ be a basis of $L$ as a $K$-linear space. We recall that $\mathcal{B}^{*}:=\left\{e_{1}^{*}, \ldots, e_{N}^{*}\right\}$ is the dual basis of $\mathcal{B}$ if $\operatorname{Tr}_{K}^{L}\left(e_{i} e_{j}^{*}\right)=1$ for $i=j$ and 0 otherwise.

Lemma 3.5 Let $\omega \in \overline{\mathbb{Q}}$ be a primitive $p$-root of 1 for some prime $p$. Then the basis $\mathcal{B}^{*}:=$ $\left\{\left(\omega^{-j}-\omega\right) / p: j=0, \ldots, p-2\right\}$ of $\mathbb{Q}(\omega)$ is dual to $\mathcal{B}:=\left\{\omega^{i}: i=0, \ldots, p-2\right\}$. 
Proof.- A direct computation shows that for $i, j=0, \ldots, p-2$

$$
\operatorname{Tr}\left(\omega^{i}\left(\omega^{-j}-\omega\right)\right)=\sum_{l=1}^{p-1} \omega^{l i}\left(\omega^{-l j}-\omega^{l}\right)=\left\{\begin{array}{lll}
p, & \text { for } & i=j, \\
0, & \text { for } & i \neq j .
\end{array}\right.
$$

We will use this result in the following way: let $\omega$ be a primitive $p$-root of 1 and set $L:=K(\omega)$. Let us assume that $\mathbb{Q}(\omega)$ and $K$ are linearly independent and that $p$ does not divide the discriminant of $K$. Both conditions are satisfied by all but a finite number of $p$. Then $[L: K]=p-1$ and $\mathcal{O}_{L}=\mathcal{O}_{K}[\omega]$ [33, Ch. III, Prop. 17].

Now, let $\nu \in L \backslash\{0\}$. Then

$$
\nu=\frac{1}{p} \operatorname{Tr}(\nu(1-\omega))+\cdots+\frac{1}{p} \operatorname{Tr}\left(\nu\left(\omega^{2-p}-\omega\right)\right) \omega^{2-p} \in K[\omega] \backslash\{0\}
$$

and so there exists $0 \leq j \leq p-2$ such that $\operatorname{Tr}\left(\nu\left(\omega^{-j}-\omega\right)\right) / p \in K \backslash\{0\}$.

Moreover, if $\nu \in \mathcal{O}_{L} \backslash\{0\}$, as every coefficient belongs to $\mathcal{O}_{K}$, there exists $0 \leq j \leq p-2$ such that $\operatorname{Tr}\left(\nu\left(\omega^{-j}-\omega\right)\right) / p \in \mathcal{O}_{K} \backslash\{0\}$.

Theorem 3.6 (Effective arithmetic Nullstellensatz)

Let $K$ be a number field and let $f_{1}, \ldots, f_{s} \in \mathcal{O}_{K}\left[x_{1}, \ldots, x_{n}\right]$ be polynomials without common zeros in $\mathbb{I A}^{n}$. Set $d:=\max _{i} \operatorname{deg} f_{i}$ and $h:=h\left(f_{1}, \ldots, f_{s}\right)$.

Then there exist $a \in \mathcal{O}_{K} \backslash\{0\}$ and $g_{1}, \ldots, g_{s} \in \mathcal{O}_{K}\left[x_{1}, \ldots, x_{n}\right]$ such that

- $a=g_{1} f_{1}+\cdots+g_{s} f_{s}$,

- $\operatorname{deg} g_{i} \leq 4 n d^{n}$,

- $h\left(a, g_{1}, \ldots, g_{s}\right) \leq 4 n(n+1) d^{n}(h+\log s+(n+7) \log (n+1) d)$.

The extremal cases $n=1$ and $d=1$ are treated directly in the following results.

Lemma 3.7 Let $\ell_{1}, \ldots, \ell_{s} \in \mathcal{O}_{K}\left[x_{1}, \ldots, x_{n}\right]$ be polynomials of degree bounded by 1 without common zeros in $\mathbb{I}^{n}$. Set $h:=h\left(\ell_{1}, \ldots, \ell_{s}\right)$.

Then there exist $a \in \mathcal{O}_{K} \backslash\{0\}$ and $a_{1}, \ldots, a_{s} \in \mathcal{O}_{K}$ such that

- $a=a_{1} \ell_{1}+\cdots+a_{s} \ell_{s}$,

- $h\left(a, a_{1}, \ldots, a_{s}\right) \leq(n+1)(h+\log (n+1))$.

Proof.- Equation $a=a_{1} \ell_{1}+\cdots+a_{s} \ell_{s}$ is equivalent to a $\mathcal{O}_{K}$-linear system of $n+1$ equations in $s$ unknowns, which can be solved applying Cramer rule. The integer $a$ is the determinant of a non-singular submatrix of the system.

Lemma 3.8 Let $f_{1}, \ldots, f_{s} \in \mathcal{O}_{K}[x]$ be polynomials without common zeros in $\mathbb{I A}^{1}$. Set $d:=$ $\max _{i} \operatorname{deg} f_{i}$ and $\left.h:=h\left(f_{1}, \ldots, f_{s}\right)\right)$.

Then there exist $a \in \mathcal{O}_{K} \backslash\{0\}$ and $g_{1}, \ldots, g_{s} \in \mathcal{O}_{K}[x]$ such that

- $a=g_{1} f_{1}+\cdots+g_{s} f_{s}$,

- $\operatorname{deg} g_{i} \leq d-1$,

- $h\left(a, g_{1}, \ldots, g_{s}\right) \leq 2 d(h+d)$. 
Proof.- Let $f:=\sum_{i} a_{i} f_{i}, g:=\sum_{i} b_{i} f_{i} \in K[x]$ be generic linear combinations of $f_{1}, \ldots, f_{s}$. Then $f$ and $g$ are coprime polynomials, and so there exist $p, q \in K[x]$ with $\operatorname{deg} p<\operatorname{deg} g$ and $\operatorname{deg} q<\operatorname{deg} f$ such that $1=p f+q g$.

Expanding this identity there exists $p_{1}, \ldots, p_{s} \in K[x]$ with $\operatorname{deg} p_{i} \leq d-1$ such that

$$
1=p_{1} f_{1}+\cdots+p_{s} f_{s} .
$$

Thus the above Bézout identity translates to a consistent system of $K$-linear equations. The number of equations and variables equal $2 d$ and $s d$ respectively. This system can be solved by Cramer rule. The integer $a$ is the determinant of a non-singular $2 d \times 2 d$-submatrix of the matrix of the linear system.

Proof of Theorem 1.- We assume $n>1$ and $d>1$.

Let $G_{p} \subset \overline{\mathbb{Q}}$ denote the group of $p$-roots of 1 , for a prime $p$. For $a_{i j} \in G_{p}$ and $i=1, \ldots, \min \{n+$ $1, s\}$ we set

$$
q_{i}:=a_{i 1} f_{1}+\ldots+a_{i s} f_{s} .
$$

Also, for $b_{k l} \in G_{p}$ and $k=1, \ldots, n$ we set

$$
y_{k}:=b_{k 0}+b_{k 1} x_{1}+\cdots+b_{k n} x_{n} .
$$

We will assume that for a specific choice of $p, a_{i j}$ and $b_{k l}$ there exists $t \leq \min \{n+1, s\}$ such that $\left(q_{1}, \ldots, q_{i}\right) \subset K\left[x_{1}, \ldots, x_{n}\right]$ is a radical ideal of dimension $n-i$ for $i=1, \ldots, t-1$ and $1 \in\left(q_{1}, \ldots, q_{t}\right)$. We also assume that $y_{1}, \ldots, y_{n}$ is a linear change of variables, and that $V_{i}:=$ $V\left(q_{1}, \ldots, q_{i}\right) \subset \mathbb{I}^{n}$ satisfies Assumption 1.5 for $i=1, \ldots, t-1$ with respect to $y_{1}, \ldots, y_{n-i}$.

This is guaranteed by the fact that these conditions are generically satisfied: there exists a hypersurface $H$ of the coefficient space such that $\left(a_{i j}, b_{k l}\right) \notin H$ implies that $q_{1}, \ldots, q_{s}$ satisfy the stated conditions with respect to the variables $y_{1}, \ldots, y_{n}$ 17, 477. As $\cup_{p} G_{p}$ is Zariski dense in $I^{1}$, it follows that these coefficients can be chosen to lie in $G_{p}$ for some $p$. Moreover, $p$ can be chosen such that for $\omega$ a primitive $p$-root of 1 and $L:=K(\omega), \mathbb{Q}(\omega)$ and $K$ are linearly independent and $p$ does not divide the discriminant of $K$.

We refer the reader to Section 4.1, where we give a self-contained treatment of this topic.

Set $b:=\left(b_{k 0}\right)_{k} \in G_{p}^{n}$ and $B:=\left(b_{k l}\right)_{k, l \geq 1} \in \mathrm{GL}_{n}(\overline{\mathbb{Q}})$. For $j=1, \ldots, t$ set

$$
F_{j}(y):=q_{j}(x)=q_{j}\left(B^{-1}(y-b)\right) \in L\left[y_{1}, \ldots, y_{n}\right] .
$$

Then $F_{1}, \ldots, F_{t}$ satisfy the hypothesis of Corollary 3.4. Let $\gamma \in L^{*}$ and $P_{1}, \ldots, P_{t} \in L\left[y_{1}, \ldots, y_{n}\right]$ be the non-zero element and the polynomials satisfying Bézout identity we obtain there.

Now, for $i=1, \ldots, s$, set

$$
p_{i}:=\sum_{j=1}^{t} a_{i j} P_{j}(B x+b) \in L\left[x_{1}, \ldots, x_{n}\right]
$$

so that $1=p_{1} f_{1}+\cdots+p_{s} f_{s}$ holds.

Finally set $\mu:=(\operatorname{det} B)^{4 n(n+1) d^{n+1}} \gamma \in L^{*}$. By Lemma 3.5 there exists $0 \leq \ell \leq p-2$ such that $\operatorname{Tr}\left(\mu\left(\omega^{-\ell}-\omega\right)\right) \neq 0$.

We define

$$
a:=\operatorname{Tr}\left(\mu\left(\omega^{-\ell}-\omega\right)\right) / p \in K^{*} \quad, \quad g_{i}:=\operatorname{Tr}\left(\mu p_{i}\left(\omega^{-\ell}-\omega\right)\right) / p \in K\left[x_{1}, \ldots, x_{n}\right]
$$

for $i=1, \ldots, s$.

Then

$$
a=g_{1} f_{1}+\cdots+g_{s} f_{s}
$$


as $f_{1}, \ldots, f_{s} \in K\left[x_{1}, \ldots, x_{n}\right]$ and $\operatorname{Tr}$ is a $K$-linear map.

Aside from the degree and height bounds, we will show that since $f_{1}, \ldots, f_{s} \in \mathcal{O}_{K}\left[x_{1}, \ldots, x_{n}\right]$, $a \in \mathcal{O}_{K}$ and $g_{i} \in \mathcal{O}_{K}\left[x_{1}, \ldots, x_{n}\right]$.

Let us first analyze degrees and local heights.

As $\operatorname{deg} F_{j} \leq d, \operatorname{deg} g_{i} \leq \operatorname{deg} p_{i} \leq \max _{j} \operatorname{deg} P_{j} \leq 4 n d^{n}$.

Now let $v \in M_{K}^{\infty}$ and let $w \in M_{L}$ such that $w \mid v$. We have $h_{w}\left(B^{-1}(y-b)\right) \leq n \log n-$ $\log |\operatorname{det} B|_{w}$ and so

$$
\begin{aligned}
h_{w}\left(F_{j}\right) & \leq h_{w}\left(q_{j}\right)+\left(n \log n-\log |\operatorname{det} B|_{w}+2 \log (n+1)\right) d \\
& \leq h_{v}+\log s+(n+2) \log (n+1) d-\log |\operatorname{det} B|_{w} d
\end{aligned}
$$

by Lemma 1.2(c). From Corollary 3.4

$$
\begin{aligned}
h_{w}\left(P_{j}\right) \leq & 4 n(n+1) d^{n} \max _{k} h_{w}\left(F_{k}\right)+4 n(4 n+5) \log (n+1) d^{n+1}-\log |\gamma|_{w} \\
\leq & 4 n(n+1) d^{n}\left(h_{v}+\log s+(n+2) \log (n+1) d-\log |\operatorname{det} B|_{w} d\right) \\
& +4 n(4 n+5) \log (n+1) d^{n+1}-\log |\gamma|_{w} \\
= & 4 n(n+1) d^{n}\left(h_{v}+\log s\right)+4 n\left(n^{2}+7 n+7\right) \log (n+1) d^{n+1}-\log |\mu|_{w} .
\end{aligned}
$$

Therefore

$$
\begin{aligned}
h_{w}\left(\mu p_{i}\right) \leq & \max _{j} h_{w}\left(P_{j}\right)+2 \log (n+1) \max _{j} \operatorname{deg} P_{j}+\log t+\log |\mu|_{w} \\
\leq & 4 n(n+1) d^{n}\left(h_{v}+\log s\right)+4 n\left(n^{2}+7 n+7\right) \log (n+1) d^{n+1} \\
& +8 n \log (n+1) d^{n}+\log (n+1) \\
\leq & 4 n(n+1) d^{n}\left(h_{v}+\log s+(n+7) \log (n+1) d\right)-\log 2
\end{aligned}
$$

again by Lemma 1.2 (c) and the fact $d, n \geq 2$. We have

and so

$$
g_{i}=\frac{1}{p} \operatorname{Tr}\left(\mu p_{i}\left(\omega^{-\ell}-\omega\right)\right)=\frac{1}{p} \sum_{\sigma \in \mathrm{Gal}_{L / K}} \sigma\left(\mu p_{i}\left(\omega^{-\ell}-\omega\right)\right)
$$

$$
\begin{aligned}
h_{v}\left(g_{i}\right) & \leq \max _{w \mid v} h_{w}\left(\mu p_{i}\right)+\log 2 \\
& \leq 4 n(n+1) d^{n}\left(h_{v}+\log s+(n+7) \log (n+1) d\right) .
\end{aligned}
$$

We have $h_{w}(\mu) \leq 4 n(n+1) d^{n}\left(h_{v}+\log s\right)+4 n\left(n^{2}+7 n+7\right) \log (n+1) d^{n+1}$ and so the previous estimate also holds for $h_{v}(a)$.

Now let $v \notin M_{K}^{\infty}$ and $w \mid v$. Analogously we have

$$
h_{w}(\mu), h_{v}\left(\mu p_{i}\right) \leq 4 n(n+1) d^{n} h_{v}=0
$$

as $f_{1}, \ldots, f_{s} \in \mathcal{O}_{K}\left[x_{1}, \ldots, x_{n}\right]$. Then $\mu \in \mathcal{O}_{L} \backslash\{0\} \quad$ and $\mu p_{i} \in \mathcal{O}_{L}\left[x_{1}, \ldots, x_{n}\right]$, which in term implies that $a \in \mathcal{O}_{K} \backslash\{0\}$ and $g_{i} \in \mathcal{O}_{K}\left[x_{1}, \ldots, x_{n}\right]$ as desired.

The global height estimate follows then from the expression

$$
h\left(a, g_{1}, \ldots, g_{s}\right)=\frac{1}{[K: \mathbb{Q}]} \sum_{v \in M_{K}^{\infty}} N_{v} \max \left\{h_{v}(a), h_{v}\left(g_{1}\right), \ldots, h_{v}\left(g_{s}\right)\right\} .
$$


Remark 3.9 The fact that the bound 3.0 is uniform on $w$ for $w \mid v$ is the key that allows us to get rid of the roots of 1 . This will no longer be the case in our treatment of the more refined arithmetic Nullstellensätze in Chapter 4.

The following example, although not verifying the worst case bound for the degrees, improves the lower bound stated in the introduction for a general height estimate and shows that the term $d^{n} h$ is unavoidable.

Example 3.10 Set

$$
f_{1}:=x_{1}-H, \quad f_{2}:=x_{2}-x_{1}^{d}, \ldots, \quad f_{n}:=x_{n}-x_{n-1}^{d}, \quad f_{n+1}:=x_{n}^{d}
$$

for any $d, H \in \mathbb{N}$. These are polynomials without common zeros in $\mathbb{I}^{n}$ of degree and height bounded by $d$ and $h:=\log H$ respectively.

Let $a \in \mathbb{Z} \backslash\{0\}$ and $g_{1}, \ldots, g_{n+1} \in \mathbb{Z}\left[x_{1}, \ldots, x_{n}\right]$ such that $a=g_{1} f_{1}+\cdots+g_{n+1} f_{n+1}$. We evaluate this identity in $\left(H, H^{d}, \cdots, H^{d^{n-1}}\right)$ and we obtain

$$
a=g_{n+1}\left(H, H^{d}, \cdots, H^{d^{n-1}}\right) H^{d^{n}}
$$

from where we deduce $h(a) \geq d^{n} h$.

\section{Intrinsic type estimates}

Theorem 1 is essentially optimal in the general case. There are however many particular instances in which these estimates can be improved. Consider the following example:

$$
f_{1}:=x_{1}-1, \quad f_{2}:=x_{2}-x_{1}^{d}, \ldots, \quad f_{n}:=x_{n}-x_{n-1}^{d}, \quad f_{n+1}:=H-x_{n}^{d}
$$

for any $d, H \in \mathbb{N}$. These are polynomials without common zeros in $\mathbb{I A}^{n}$ of degree and height bounded by $d$ and $h:=\log H$ respectively. Theorem 1 says there exist $a \in \mathbb{Z} \backslash\{0\}$ and $g_{1}, \ldots, g_{n+1} \in \mathbb{Z}\left[x_{1}, \ldots, x_{n}\right]$ such that

$$
a=g_{1} f_{1}+\cdots+g_{n+1} f_{n+1}
$$

with $\operatorname{deg} g_{i} \leq 4 n d^{n}$ and $h(a), h\left(g_{i}\right) \leq 4 n(n+1) d^{n}(h+(n+7) \log (n+1) d)$. However the following Bézout identity holds:

$$
H-1=\frac{x_{1}^{d}-1}{x_{1}-1} \cdots \frac{x_{n}^{d}-1}{x_{n}-1} f_{1}+\cdots+\frac{x_{n}^{d}-1}{x_{n}-1} f_{n}+f_{n+1} .
$$

Note that the polynomials arising in this identity have degree and height bounded by $n(d-1)$ and $h$ respectively.

There is in this case an exponential gap between the a priori general estimates and the actual ones. The explanation is somewhat simple: for $i=1, \ldots, n$, the varieties

$$
V_{i}:=V\left(f_{1}, \ldots, f_{i}\right)=V\left(x_{1}-1, x_{2}-1, \ldots, x_{i}-1\right) \subset \mathbb{A}^{n},
$$

verify $\operatorname{deg}\left(V_{i}\right)=1$ and $h\left(V_{i}\right) \leq 2 n \log (n+1)$. Namely, both the degree and the height of the varieties successively cut out by the input polynomials are much smaller than the corresponding Bézout estimate.

As the varieties $V_{i}$ verify the assumptions of Lemma 3.3, a direct application together with Lemma 1.3 produces the more realistic estimates:

$$
\operatorname{deg} g_{i} \leq 2 n^{2} d \quad, \quad h(a), h\left(g_{i}\right) \leq(n+1)^{2}(h+8 n \log (n+1) d) .
$$

Based on this idea, we devote this chapter to the study of more refined arithmetic Nullstellensätze which can deal with such situations. 


\subsection{Equations in general position}

This section deals with the preparation of the input data. To apply Lemma 3.3, we need to prepare the polynomials and the variables of the ambient space.

Let $f_{1}, \ldots, f_{s} \in K\left[x_{1}, \ldots, x_{n}\right]$ be polynomials without common zeros in $\mathbb{I}^{n}$. For $i=1, \ldots, s$ and $a_{i j} \in \mathbb{Z}$ we set

$$
q_{i}:=a_{i 1} f_{1}+\cdots+a_{i s} f_{s} .
$$

We will estimate the height of rational integers $a_{i j}$ in order that there exists $t \leq \min \{n+1, s\}$ such that $\left(q_{1}, \ldots, q_{i}\right) \subset K\left[x_{1}, \ldots, x_{n}\right]$ is a radical ideal of dimension $n-i$ for $i=1, \ldots, t-1$ and $1 \in\left(q_{1}, \ldots, q_{t}\right)$.

Also we set

$$
y_{k}:=b_{k 0}+b_{k 1} x_{1}+\cdots+b_{k n} x_{n}
$$

for $k=1, \ldots, n$ and $b_{k l} \in \mathbb{Z}$. Again we want to estimate the height of rational integers $b_{k l}$ such that $V_{i}:=V\left(q_{1}, \ldots, q_{i}\right) \subset \mathbb{A}^{n}$ satisfies Assumption 1.5 with respect to this set of variables for $i=1, \ldots, t-1$. Namely, the projection

$$
\pi_{i}: V_{i} \rightarrow \mathbb{A}^{n-i} \quad, \quad x \mapsto\left(y_{1}, \ldots, y_{n-i}\right)
$$

must verify $\# \pi_{i}^{-1}(0)=\operatorname{deg} V_{i}$, that is $\# V_{i} \cap V\left(y_{1}, \cdots, y_{n-i}\right)=\operatorname{deg} V_{i}$ for $i=1, \ldots, t-1$. Lemma 2.13 implies that the variables $y_{1}, \ldots, y_{n-i}$ are in Noether normal position with respect to $V_{i}$.

It is well-known that these conditions are satisfied by a generic election of $a_{i j}$ and $b_{k l}$, see for instance 17, 47].

We have already applied such a preparation to obtain the classic style version of the effective arithmetic Nullstellensatz presented in Theorem 3.6. There, we chose roots of 1 as coefficients of the linear combinations since their existence was sufficient in our proof. However, technical reasons (see Remark 3.9) prevent us to apply the same principle in this chapter, and we need to carry out a more careful analysis.

We note that all aspects of this preparation were previously covered in the research papers [2], [17], [29], [19]. However the bounds presented therein are either non-explicit or not precise enough for our purposes. Here we chose to give a self-contained presentation, which yields another proof of the existence of such linear combinations, with good control of the integers height.

\subsubsection{An effective Bertini theorem}

This subsection concerns the preparation of the polynomials. We will first show some auxiliary results.

The following is a version of the so-called shape lemma representation of a 0-dimensional radical ideal. The main difference here is that we choose a generic linear form - instead of a particular one - as a primitive element.

For a polynomial $f=c_{D} t^{D}+\cdots+c_{0} \in k[t]$ we denote its discriminant by $\operatorname{discr}(f) \in k$. We recall that $\operatorname{discr}(f) \neq 0$ if and only if $c_{D} \neq 0$ and $f$ is squarefree, that is when $f$ has exactly $D$ distinct roots.

Lemma 4.1 (Shape Lemma)

Let $V \subset \mathbb{I A}^{n}$ be a 0-dimensional variety defined over $k$. Let $U:=\left(U_{0}, \ldots, U_{n}\right)$ be a group of $n+1$ variables, and set $L:=U_{0}+U_{1} x_{1}+\cdots+U_{n} x_{n}$ for the associated generic linear form.

Let $P:=P_{V} \in k[U][T]$ be a characteristic polynomial of $V$. Set $P^{\prime}:=\partial P / \partial T \in k[U][T]$ and $\rho:=\operatorname{discr}_{T} P \in k[U] \backslash\{0\}$. Also set $I$ for the extension of $I(V)$ to $k[U][x]$.

Then there exist $v_{1}, \ldots, v_{n} \in k[U][T]$ with $\operatorname{deg} v_{i} \leq \operatorname{deg} V-1$ such that

$$
I_{\rho}=\left(P(L), P^{\prime}(L) x_{1}-v_{1}(L), \ldots, P^{\prime}(L) x_{n}-v_{n}(L)\right)_{\rho} \subset k[U]_{\rho}[x] .
$$


Proof.- We note first that $I(V)$ is a radical ideal, and so $I=k[U] \otimes_{k} I(V)$ is also radical. We readily obtain from the definition of $P:=P_{V}$ that $I \cap k[U][L]=(P(L))$, and so $P(L) \in I$.

We can write $P(L)=\sum_{\alpha} a_{\alpha}(x) U^{\alpha}$ with $a_{\alpha}(x) \in I(V)$. Therefore $\partial P(L) / \partial U_{i}$ also lies in $I$ for all $i$. A direct computation shows that for $i=1, \ldots, n$

$$
\partial P(L) / \partial U_{i}=P^{\prime}(L) x_{i}-v_{i}(L)
$$

for some $v_{i} \in k[U][T]$ with $\operatorname{deg} v_{i} \leq \operatorname{deg} P-1=\operatorname{deg} V-1$.

Set

$$
J:=\left(P(L), P^{\prime}(L) x_{1}-v_{1}(L), \ldots, P^{\prime}(L) x_{n}-v_{n}(L)\right) \subset k[U][x] .
$$

The previous argument shows the inclusion $I \supset J$.

On the other hand, $\rho=A P+B P^{\prime}$ for some $A, B \in k[U][T]$. Set $w_{i}:=B v_{i}$. Then $x_{i} \equiv w_{i}(L) / \rho$ $\left(\bmod J_{\rho}\right)$ and so for every $f \in k[U][x]$ we have that $f \equiv f\left(U, w_{1}(L) / \rho, \ldots, w_{n}(L) / \rho\right)$ modulo $J_{\rho}$, and hence modulo $I_{\rho}$.

For $f \in I$,

$$
\rho^{\operatorname{deg} f} f\left(U, w_{1}(L) / \rho, \ldots, w_{n}(L) / \rho\right) \in I \cap k[U][L]=(P(L))
$$

which implies $I_{\rho} \subset J_{\rho}$ as desired.

Let $\nu \in k^{n+1}$ such that $\rho(\nu) \neq 0$. It follows that $I(V)$ can be represented as

$$
I(V)=\left(P(L), P^{\prime}(L) x_{1}-v_{1}(L), \ldots, P^{\prime}(L) x_{n}-v_{n}(L)\right)(\nu) \subset k[x] .
$$

Now let $f_{1}, \ldots, f_{s} \in k\left[x_{1}, \ldots, x_{n}\right]$ be polynomials without common zeros in $\mathbb{I}^{n}$. For $i=1, \ldots, s$ we let $Z_{i}:=\left(Z_{i 1}, \ldots, Z_{i s}\right)$ denote a group of $s$ variables, and we set

$$
Q_{i}:=Z_{i 1} f_{1}+\cdots+Z_{i s} f_{s} \in k[Z][x]
$$

for the associated generic linear combination of $f_{1}, \ldots, f_{s}$.

Lemma 4.2 For $\ell=1, \ldots, s$, the ideal $\left(Q_{1}, \ldots, Q_{\ell}\right)$ is a complete intersection prime ideal of $k[Z][x]$.

Proof.- Set $I:=\left(Q_{1}, \ldots, Q_{\ell}\right)$ and $V:=V(I) \subset \mathbb{I}^{s \ell} \times \mathbb{I}^{n}$. First we observe that $V$ is a linear bundle over $I^{n}$ : the projection

$$
\pi: V \rightarrow \mathbb{A}^{n} \quad, \quad(z, x) \mapsto x
$$

is surjective, and the fibers are affine spaces of dimension $(s-1) \ell$. This follows from the assumption that the $f_{j}$ have no common zeros. This implies that

$$
\operatorname{dim} V=(s-1) \ell+n
$$

because of the theorem of dimension of fibers. Namely $Q_{1}, \ldots, Q_{\ell}$ is a complete intersection, and in particular the ideal $I$ is unmixed.

Set $I=I_{1} \cap \ldots \cap I_{m}$ for the primary decomposition of this ideal. We will show that $I_{j}$ is prime for all $j$, and then that $m=1$.

First we have that $I_{f_{j}}=\left(Q_{1} / f_{j}, \ldots, Q_{\ell} / f_{j}\right)=\left(Z_{1 j}+H_{1 j}, \ldots, Z_{\ell j}+H_{\ell j}\right)$ where $H_{i j} \in k\left[Z_{i}\right][x]_{f_{j}}$ does not depend on $Z_{i j}$. Therefore

$$
\left(k\left[\mathrm{IA}^{s \ell} \times \mathbb{I}^{n}\right] / I\right)_{f_{j}} \cong k\left[\mathrm{IA}^{(s-1) \ell} \times \mathbb{I}^{n}\right]_{f_{j}}
$$


is a domain, that is $I_{f_{j}}$ is prime. We have $I_{f_{j}}=\left(I_{1}\right)_{f_{j}} \cap \ldots \cap\left(I_{m}\right)_{f_{j}}$, and so there exists $1 \leq n(j) \leq m$ such that

$$
I_{f_{j}}=\left(I_{n(j)}\right)_{f_{j}} \quad, \quad V\left(I_{i}\right) \subset\left\{f_{j}=0\right\} \quad \text { for } i \neq n(j) .
$$

In particular $I_{n(j)}=I_{f_{j}} \cap k\left[\mathbb{A}^{s \ell} \times \mathbb{A}^{n}\right]$ is prime. The fact that $\cap_{j}\left\{f_{j}=0\right\}=\emptyset$ ensures that $n(j)$ runs over all $1 \leq i \leq m$, and so $I$ is radical.

The expression $I_{f_{j}}=\left(Z_{1 j}+H_{1 j}, \ldots, Z_{\ell j}+H_{\ell j}\right)$ implies that $\pi\left(V\left(I_{f_{j}}\right)\right) \subset \mathbb{A}^{n}$ contains the dense open set $\left\{f_{j} \neq 0\right\}$. In particular $V\left(I_{f_{j}}\right)$ is not contained in any of the hypersurfaces $\left\{f_{i}=0\right\}$ and so $n(j)=n(1)$ for all $j$. This implies that $m=1$, and so $I=I_{1}$ is prime.

The following proposition shows that $\left(Q_{1}\left(a_{1}\right), \ldots, Q_{\ell}\left(a_{\ell}\right)\right)$ is a radical ideal for a generic election of $a_{i}:=\left(a_{i 1}, \ldots, a_{i s}\right)$. Unlike Lemmas 4.1 and 4.2 , this result does note hold for arbitrary characteristic. For instance, let $x^{p}, 1-x^{p} \in \mathbb{F}_{p}[x]$ for some prime $p$. Then $Q_{1}\left(a_{1}\right)=b+c x^{p}$ for some $b, c \in \overline{\mathbb{F}_{p}}$ and so

$$
Q_{1}\left(a_{1}\right)=\left(b^{1 / p}+c^{1 / p} x\right)^{p}
$$

is not squarefree.

Proposition 4.3 Let $\operatorname{char}(k)=0$ and set $I:=\left(Q_{1}, \ldots, Q_{\ell}\right) \subset k[Z][x]$.

- In case $I \cap k[Z] \neq\{0\}$ there exists $F \in k[Z] \backslash\{0\}$ with $\operatorname{deg} F \leq(d+1)^{\ell}$ such that $F\left(a_{1}, \ldots, a_{\ell}\right) \neq 0$ for $a_{1}, \ldots, a_{\ell} \in k^{s}$ implies that $1 \in\left(Q_{1}\left(a_{1}\right), \ldots, Q_{\ell}\left(a_{\ell}\right)\right)$.

- In case $I \cap k[Z]=\{0\}$ there exists $F \in k[Z] \backslash\{0\}$ with $\operatorname{deg} F \leq 2(d+1)^{2 \ell}$ such that $F\left(a_{1}, \ldots, a_{\ell}\right) \neq 0$ for $a_{1}, \ldots, a_{\ell} \in k^{s}$ implies that $\left(Q_{1}\left(a_{1}\right), \ldots, Q_{\ell}\left(a_{\ell}\right)\right) \subset k[x]$ is a radical ideal of dimension $n-\ell$.

Proof.- Set $V:=V(I) \subset \mathbb{A}^{s \ell} \times \mathbb{A}^{n}$. We have $\operatorname{dim} V=(s-1) \ell+n$ and $\operatorname{deg} V \leq(d+1)^{\ell}$.

First we consider the case $I \cap k[Z] \neq\{0\}$. This occurs, for instance, when $\ell \geq n+1$, since then $\operatorname{dim} I=s \ell+n-\ell<\operatorname{dim} k[Z]=s \ell$.

Let $\pi: I \mathrm{~A}^{s \ell} \times I \mathrm{~A}^{n} \rightarrow \mathbb{I}^{s \ell}$ be the canonical projection. Then $\overline{\pi(V)}$ is a proper subvariety of $\mathbb{I}^{s \ell}$, and thus it is contained in a hypersurface of degree bounded by $\operatorname{deg} V$. This can be seen by taking a generic projection of this variety into an affine space of dimension $s \ell+n-\ell+1$ [20, Remark 4]. Let $F \in k[Z]$ be a defining equation of this hypersurface. Then $F \in I$ as $I$ is prime, and we have $\operatorname{deg} F \leq(d+1)^{\ell}$. Thus

$$
1 \in I_{F} \subset k[Z]_{F}[x]
$$

and therefore $1 \in I(a):=\left(Q_{1}\left(a_{1}\right), \ldots, Q_{\ell}\left(a_{\ell}\right)\right)$ for $a \in k^{s \ell}$ such that $F(a) \neq 0$.

Next we consider the case $I \cap k[Z]=\{0\}$.

We adopt the following convention: for an ideal $J \subset k[x]$ and for $\zeta$ any new group of variables, we denote by $J^{[\zeta]}$ and $J^{(\zeta)}$ the extension of $J$ to the polynomial rings $k[\zeta][x]$ and $k(\zeta)[x]$ respectively.

We assume for the moment $\ell=n$. Then $\operatorname{dim} I=s \ell$ and so the extended ideal $I^{(Z)} \subset k(Z)[x]$ is a 0 -dimensional prime ideal. We have then that $\overline{k(Z)} \otimes_{k} I^{(Z)} \subset \overline{k(Z)}[x]$ is a radical ideal, as $\operatorname{char}(k)=0$ [41, Thm. 26.3].

Our approach to this case is based on Shape Lemma 4.1. We will determine a polynomial $F \in k[Z]$ such that $F(a) \neq 0$ implies that the shape lemma representation of $I^{(Z)}$ can be transferred to a shape lemma representation of $I(a)$.

Let $U$ be a group of $n+1$ variables and set

$L:=U_{0}+U_{1} x_{1}+\cdots+U_{n} x_{n}$ for the associated generic linear form. Consider the morphism

$$
\Psi: \mathbb{A}^{n+1} \times \mathbb{A}^{s \ell} \times \mathbb{A}^{n} \rightarrow \mathbb{A}^{n+1} \times \mathbb{A}^{s \ell} \times \mathbb{A}^{1} \quad, \quad(u, z, x) \mapsto(u, z, L(x)) .
$$


and let $W$ be the variety defined by $I$ in $I \mathrm{~A}^{n+1} \times I \mathrm{~A}^{s \ell} \times I \mathrm{~A}^{n}$, that is $W=I \mathrm{~A}^{n+1} \times V$. The Zariski closure $\overline{\Psi(W)}$ is then an irreducible hypersurface. We set $P \in k[U, Z][T]$ for one of its defining equations.

If $I^{[U](Z)}$ is the extension of $I^{(Z)}$ to $k[U](Z)[x]$, the polynomial $P$ can be equivalently defined through the condition that $P(L)$ is a generator of the principal ideal $I^{[U](Z)} \cap k[U, Z][L]$. Namely, $P$ is a characteristic polynomial of the 0 - dimensional variety $W_{0}$ defined by $I^{(Z)}$ in $I^{n}(\overline{k(Z)})$.

Let $v_{1}, \ldots, v_{n} \in k[U](Z)[T]$ denote the polynomials arising in Shape Lemma applied to $W_{0}$. From the proof of this lemma we have that

$$
\partial P(L) / \partial U_{i}=P^{\prime}(L) x_{i}-v_{i}(L) \in k[U, Z][L]
$$

and so $v_{i} \in k[U, Z][T]$. Set $J:=\left(P(L), P^{\prime}(L) x_{1}-v_{1}(L), \ldots, P^{\prime}(L) x_{n}-v_{n}(L)\right) \subset k[U, Z][x]$ and $\rho:=\operatorname{discr}_{T} P \in k[U, Z] \backslash\{0\}$. Then

$$
\left(I^{[U](Z)}\right)_{\rho}=\left(J^{[U](Z)}\right)_{\rho} \subset k[U](Z)_{\rho}[x] .
$$

We have that both $I_{\rho}^{[U, Z]}$ and $J_{\rho}^{[U, Z]}$ are prime ideals of $k[U, Z]_{\rho}[x]$ with trivial intersection with the ring $k[U, Z]$. Thus they coincide with the contraction of $I_{\rho}^{[U](Z)}$ and $J_{\rho}^{[U](Z)}$ to $k[U, Z]_{\rho}[x]$ respectively, and so

$$
I_{\rho}^{[U, Z]}=J_{\rho}^{[U, Z]} \subset k[U, Z]_{\rho}[x] .
$$

Define $F \in k[Z] \backslash\{0\}$ as any of the non-zero coefficients of the monomial expansion of $\rho$ with respect to $U$. Let $a \in k^{s \ell}$ such that $F(a) \neq 0$. Then $\rho(U, a) \neq 0$ and so $P(U, a)[T]$ is squarefree. Then

$$
\left(I(a)^{[U]}\right)_{\rho(U, a)}=\left(P(L), P^{\prime}(L) x_{1}-v_{1}(L), \ldots, P^{\prime}(L) x_{n}-v_{n}(L)\right)(a) \subset k[U][x]
$$

is radical, which implies in turn that $I(a)=\left(I(a)^{[U]}\right)_{\rho(U, a)} \cap k[x]$ is a radical ideal of $k[x]$ as desired.

It remains to estimate the degree of $F$. To this end, it suffices to bound the degree of $\rho$ with respect to the group of variables $Z$. We recall that $P$ was defined as a defining equation of the hypersurface $\overline{\Psi(W)}$. The map $\Psi$ is linear in the variables $Z$ and $x$, and so

$$
\operatorname{deg}_{Z} P \leq \operatorname{deg} W=\operatorname{deg} V \leq(d+1)^{n} .
$$

This implies that $\operatorname{deg} F \leq \operatorname{deg}_{Z} \rho \leq \operatorname{deg}_{Z} P\left(2 \operatorname{deg}_{Z} P-1\right) \leq 2(d+1)^{2 n}$.

Finally we consider the case $\ell<n$ for $I \cap k[Z]=\{0\}$.

Let $U_{1}, \ldots, U_{n-\ell}$ be groups of $n+1$ variables each, and set

$$
L_{i}:=U_{i 0}+U_{i 1} x_{1}+\cdots+U_{i n} x_{n} .
$$

for $i=1, \ldots, n-\ell$. Set $U:=\left(U_{1}, \ldots, U_{n-\ell}\right), L:=\left(L_{1}, \ldots, L_{n-\ell}\right)$ and $k_{0}:=k(U, L)$. The extended ideal $I_{0} \subset k_{0}[Z]\left[x_{1}, \ldots, x_{\ell}\right]$ verifies $I_{0} \cap k_{0}[Z]=\{0\}$ and thus falls into the previously considered case.

Thus there exists $F_{0} \in k_{0}[Z] \backslash\{0\}$ with $\operatorname{deg} F_{0} \leq 2(d+1)^{2 \ell}$ such that $F_{0}(a) \neq 0$ for $a \in k^{s \ell}$ implies that $I_{0}(a)$ is a radical ideal of $k_{0}[Z]\left[x_{1}, \ldots, x_{\ell}\right]$. This implies in turn that $I(a)$ is a radical ideal of $k[x]$, as

$$
I(a)=I_{0}(a) \cap k[x] .
$$

We can assume without loss of generality that $F_{0}$ lies in $k[U, L][Z]$. We conclude by taking $F$ as any non-zero coefficient of the monomial expansion of $F_{0}$ with respect to the variables $U$ and $L$. 
Corollary 4.4 Let $\operatorname{char}(k)=0$, and let $f_{1}, \ldots, f_{s} \in k\left[x_{1}, \ldots, x_{n}\right]$ be polynomials without common zeros in $\mathbb{I A}^{n}$. Set $d:=\max _{i} \operatorname{deg} f_{i}$.

Then there exist $t \leq \min \{n+1, s\}$ and $a_{1}, \ldots, a_{t} \in \mathbb{Z}^{s}$ such that

- $\left(Q_{1}\left(a_{1}\right), \ldots, Q_{i}\left(a_{i}\right)\right)$ is a radical ideal of dimension $n-i$ for $1 \leq i \leq t-1$,

- $1 \in\left(Q_{1}\left(a_{1}\right), \ldots, Q_{t}\left(a_{t}\right)\right)$,

- $h\left(a_{i}\right) \leq 2(n+1) \log (d+1)$.

Proof.- Set $t$ for the minimal $i$ such that $I_{i}:=\left(Q_{1}, \ldots, Q_{i}\right) \cap k[Z] \neq\{0\}$. Then $t \leq n+1$, and by the previous result there exists $F_{t} \in k[Z]$ with $\operatorname{deg} F_{t} \leq(d+1)^{t}$ such that $F_{t}(a) \neq 0$ implies that $1 \in\left(Q_{1}\left(a_{1}\right), \ldots, Q_{t}\left(a_{t}\right)\right)$.

On the other hand, for $i<t$ we take a polynomial $F_{i} \in k[Z]$ of degree bounded by $2(d+1)^{2 i}$ such that $F_{i}(a) \neq 0$ implies that $\left(Q_{1}\left(a_{1}\right), \ldots, Q_{i}\left(a_{i}\right)\right)$ is a radical ideal of dimension $n-i$. Then we take $F:=F_{1} \cdots F_{t}$ and so

$$
\begin{aligned}
\operatorname{deg} F & \leq 2(d+1)^{2}+\cdots+2(d+1)^{2(t-1)}+(d+1)^{t} \\
& \leq(d+1)^{2 n}+2(d+1)^{2 n}+(d+1)^{n+1} \\
& \leq 4(d+1)^{2 n} .
\end{aligned}
$$

Finally, $F \neq 0$ implies there exist $a_{1}, \ldots, a_{t} \in \mathbb{Z}^{s}$ such that $h\left(a_{i}\right) \leq \log (\operatorname{deg} F)$ and $F(a) \neq 0$.

\subsubsection{Effective Noether normal position}

Now we devote to the preparation of the variables. For $k=0, \ldots, n$ we let $U_{k}:=\left(U_{k 0}, \ldots, U_{k n}\right)$ be a group of $n+1$ variables and we set

$$
Y_{k}:=U_{k 0}+U_{k 1} x_{1}+\cdots+U_{k n} x_{n}
$$

Proposition 4.5 Let $V \subset \mathbb{I}^{n}$ be an equidimensional variety of dimension $r$ defined over $k$.

Then there exists $G \in k\left[U_{1}, \ldots, U_{r}\right] \backslash\{0\}$ with $\operatorname{deg}_{U_{k}} G \leq 2(\operatorname{deg} V)^{2}$ such that $G\left(b_{1}, \ldots, b_{r}\right) \neq 0$ for $b_{1}, \ldots, b_{r} \in k^{n+1}$ implies that

$$
\# V \cap V\left(Y_{1}\left(b_{1}\right), \ldots, Y_{r}\left(b_{r}\right)\right)=\operatorname{deg} V .
$$

Proof.- Let $f_{V}$ be a Chow form of $V$ and $P_{V} \in k[U, T]$ be the characteristic polynomial of $V$ associated to $f_{V}$ given by Lemma 2.12 .

Set $D:=\operatorname{deg} V$ and let $P_{V}=c_{D} T_{0}^{D}+\cdots+c_{0}$ be its expansion with respect to $T_{0}$. Also set

$$
\rho:=\operatorname{discr}_{T_{0}} P_{V} \in k\left[U_{0}, \ldots, U_{r}\right]\left[T_{1}, \ldots, T_{r}\right] \backslash\{0\}
$$

for the discriminant of $P_{V}$ with respect to $T_{0}$.

Observe that as $P_{V}$ is multihomogeneous of degree $D$ in each group of variables $U_{i} \cup\left\{T_{i}\right\}$, the degree of $\rho$ in each of these group of variables is bounded by $D(2 D-1)$.

Now let $\nu_{1}, \ldots, \nu_{r} \in \bar{k}^{n+1}$ such that $V(\nu):=V \cap V\left(Y_{1}\left(\nu_{1}\right), \ldots, Y_{r}\left(\nu_{r}\right)\right)$ is a 0 -dimensional variety of cardinality $D$, and $f_{V(\nu)}$ be a Chow forms of $V(\nu)$.

Set $\zeta_{0}:=\left(T_{0}-U_{00}, U_{01} \ldots, U_{0 n}\right)$. Then applying [44, Prop. 2.4], there exists $\lambda \in k^{*}$ such that:

$P_{V}\left(U_{0}, \nu_{1}, \ldots, \nu_{r}\right)\left(T_{0}, 0, \ldots, 0\right)=f_{V}\left(\zeta_{0}\left(U_{0}, T_{0}\right), \nu_{1}, \ldots, \nu_{r}\right)=\lambda f_{V(\nu)}\left(\zeta_{0}\left(U_{0}, T_{0}\right)\right)=\lambda P_{V(\nu)}\left(U_{0}\right)\left(T_{0}\right)$ 
where $P_{V(\nu)}$ is a characteristic polynomial of $V(\nu)$.

This implies $P_{V}(U)\left(T_{0}, 0, \ldots, 0\right) \in k[U]\left[T_{0}\right]$ is a squarefree polynomial and so $\rho(U)(0) \neq 0$.

We take $G \in k\left[U_{1}, \ldots, U_{r}\right]$ as any non-zero coefficient of the expansion of $\rho(U)(0)$ with respect to $U_{0}$. Therefore

$$
\operatorname{deg} G \leq \operatorname{deg}_{U_{i}} \rho(U)(0) \leq D(2 D-1) .
$$

The condition $G(b) \neq 0$ implies that $\rho\left(U_{0}, b_{1}, \ldots, b_{r}\right)(0) \neq 0$, and so $\# V(b)=D$.

As we noted before, this implies that the variables $Y_{1}\left(b_{1}\right), \ldots, Y_{r}\left(b_{r}\right)$ are in Noether normal position with respect to the variety $V$.

Corollary 4.6 Let char $(k)=0$ and let $q_{1}, \ldots, q_{t} \in k\left[x_{1}, \ldots, x_{n}\right]$ be polynomials without common zeros in $I^{n}$ which form a reduced weak regular sequence. Set $d:=\max _{i} \operatorname{deg} f_{i}$.

Then there exist $b_{1}, \ldots, b_{n} \in \mathbb{Z}^{n+1}$ such that for $i=1, \ldots, t, V\left(q_{1}, \ldots, q_{i}\right)$ satisfies Assumption 1.5 with respect to the variables $Y_{1}\left(b_{1}\right), \ldots, Y_{n-i}\left(b_{n-i}\right)$ and

$$
h\left(b_{k}\right) \leq 2(n+1) \log (d+1) .
$$

Proof.- This follows readily from the previous result. We take $G_{i}$ as the polynomial corresponding to the variety $V\left(q_{1}, \ldots, q_{i}\right)$ and we set $G:=G_{1} \cdots G_{t-1} \in k\left[U_{1}, \ldots, U_{n}\right]$. We have $\operatorname{deg}_{U_{j}} G_{i} \leq$ $2 d^{2 i}$ and so

$$
\operatorname{deg}_{U_{j}} G \leq 2 d^{2}+\cdots+2 d^{2(t-1)} \leq 4 d^{2(t-1)} \leq 4 d^{2 n} .
$$

We conclude by taking $b_{1}, \ldots, b_{n} \in \mathbb{Z}^{n+1}$ such that $h\left(b_{i}\right) \leq \log (\operatorname{deg} G)$ and $G(b) \neq 0$.

\subsection{An intrinsic arithmetic Nullstellensatz}

In this section we introduce the notions of degree and height of a polynomial system defined over a number field $K$. Modulo setting the input equations in general position, these parameters measure the degree and height of the varieties successively cut out.

The resulting estimates for the arithmetic Nullstellensatz are linear in these parameters.

As an important particular case, we derive a sparse arithmetic Nullstellensatz.

\subsubsection{Intrinsic parameters}

Let $f_{1}, \ldots, f_{s} \in K\left[x_{1}, \ldots, x_{n}\right]$ be polynomials of degree bounded by $d$ without common zeros in $I^{n}$. For $i=1, \ldots, s$ we let $Z_{i}$ denote a group of $s$ variables and we set

$$
Q_{i}(Z):=Z_{i 1} f_{1}+\cdots+Z_{i s} f_{s} \in K[Z][x]
$$

for the associated generic linear combination of $f_{1}, \ldots, f_{s}$.

Let $\Gamma$ be the set of integer $s \times s$-matrices $a=\left(a_{i j}\right)_{i j} \in \mathbb{Z}^{s \times s}$ of height bounded by $2(n+$ 1) $\log (d+1)$ such that

$$
I_{i}(a):=\left(Q_{1}\left(a_{1}\right), \ldots, Q_{i}\left(a_{i}\right)\right) \subset K\left[x_{1}, \ldots, x_{n}\right]
$$

is a radical ideal of dimension $n-i$ for $i=1, \ldots, t-1$ and $1 \in I_{t}(a)$ for some $t \leq \min \{n+1, s\}$. Corollary 4.4 implies that $\Gamma \neq \emptyset$.

For $a \in \Gamma$ we set

$$
\begin{aligned}
\delta(a) & :=\max \left\{\operatorname{deg} V\left(I_{i}(a)\right) ; 1 \leq i \leq \min \{t, n\}-1\right\}, \\
\eta(a) & :=\max \left\{h\left(V\left(I_{i}(a)\right)\right) ; 1 \leq i \leq t-1\right\} .
\end{aligned}
$$

We set $\Gamma_{\text {min }} \subset \mathbb{Z}^{s \times s}$ for the subset of matrices $a \in \Gamma$ such that $\eta(a)+d \delta(a)$ is minimum. Finally let $a_{\min } \in \Gamma_{\min }$ be a matrix which attains the minimum of $\delta(a)$ for $a \in \Gamma_{\min }$. 
Definition 4.7 Let notations be as in the previous paragraph. Then we define the degree and the height of the polynomial system $f_{1}, \ldots, f_{s}$ respectively as

$$
\delta\left(f_{1}, \ldots, f_{s}\right):=\delta\left(a_{\min }\right) \quad, \quad \eta\left(f_{1}, \ldots, f_{s}\right):=\eta\left(a_{\min }\right) .
$$

We restrict ourselves to integer matrices of bounded height in order to keep control of the height of $Q_{1}\left(a_{1}\right), \ldots, Q_{t}\left(a_{t}\right)$. The election of $\eta(a)+d \delta(a)$ as the defining invariant comes from the need of estimating the degree and height simultaneously.

We note that in case $f_{1}, \ldots, f_{s}$ is already a reduced weak regular sequence we have

$$
\eta\left(f_{1}, \ldots, f_{s}\right)+d \delta\left(f_{1}, \ldots, f_{s}\right) \leq \eta(\mathrm{Id})+d \delta(\mathrm{Id}) .
$$

We can estimate this parameters through the arithmetic Bézout inequality:

Lemma 4.8 Let $f_{1}, \ldots, f_{s} \in K\left[x_{1}, \ldots, x_{n}\right]$ be polynomials without common zeros in $\mathbb{I A}^{n}$. Set $d_{i}:=\operatorname{deg} f_{i}$ and assume that $d_{1} \geq \cdots \geq d_{s}$ holds. Set $d:=d_{1}=\max _{i} \operatorname{deg} f_{i}$ and $h:=$ $h\left(f_{1}, \ldots, f_{s}\right)$. Also set $n_{0}:=\min \{n, s\}$ and $n_{1}:=\min \{n+1, s\}$. Then

- $\delta\left(f_{1}, \ldots, f_{s}\right) \leq \prod_{j=1}^{n_{0}-1} d_{j}$,

- $\eta\left(f_{1}, \ldots, f_{s}\right) \leq n \prod_{j=1}^{n_{1}-2} d_{j}(h+\log s+3 n(n+1) d)$.

Proof.- Let $a:=a_{\min }=\left(a_{i j}\right)_{i j} \in \mathbb{Z}^{s \times s}$ be the coefficient matrix which realizes the degree and height of $f_{1}, \ldots, f_{s}$ and set

$$
q_{i}:=a_{i 1} f_{1}+\cdots+a_{i s} f_{s}, \quad 1 \leq i \leq s .
$$

Let $t \leq n_{1}=\min \{n+1, s\}$ be minimum such that $1 \in\left(q_{1}, \ldots, q_{t}\right)$. Let $\widetilde{a} \in \mathbb{Z}^{(t-1) \times s}$ be the matrix formed by the first $t-1$ rows of $a$ and let $c \in \mathbb{Z}^{(t-1) \times s}$ be a staircase matrix equivalent to $\widetilde{a}$.

The polynomial system

$$
\widetilde{q}_{i}:=c_{i 1} f_{1}+\cdots+c_{i s} f_{s}
$$

is then equivalent to $q_{1}, \ldots, q_{t-1}$, that is $\left(\widetilde{q}_{1}, \ldots, \widetilde{q}_{i}\right)=\left(q_{1}, \ldots, q_{i}\right)$ for $i=1, \ldots, t-1$. Also we have $\operatorname{deg} \widetilde{q}_{i} \leq d_{i}$, and so

$$
\delta:=\max \left\{\operatorname{deg} V_{i} ; 1 \leq i \leq \min \{n, t\}-1\right\} \leq \prod_{j=1}^{n_{0}-1} d_{j} .
$$

We have also that each coefficient of $c$ is a subdeterminant of $\widetilde{a}$. Thus

$$
\begin{aligned}
\widetilde{h}:=h\left(\widetilde{q}_{1}, \ldots, \widetilde{q}_{t-1}\right) & \leq h+\log s+h(c) \\
& \leq h+\log s+(t-1)(2(n+1) \log (d+1)+\log (t-1)) \\
& \leq h+\log s+n(3 n+1) d
\end{aligned}
$$

and so, applying Corollary 2.10,

$$
\begin{aligned}
\eta & \leq \max \left\{h\left(V_{i}\right): 1 \leq i \leq \min \{n+1, t\}-1\right\} \\
& \left.\leq\left(\prod_{j=1}^{n_{1}-1} d_{j}\right)\left(\sum_{j=1}^{n_{1}-1} \widetilde{h}\right) / d_{j}+\left(n+n_{1}-1\right) \log (n+1)\right) \\
& \leq\left(\prod_{j=1}^{n_{1}-2} d_{j}\right)(n(h+\log s+n(3 n+1) d)+2 n \log (n+1)) \\
& \leq n\left(\prod_{j=1}^{n_{1}-2} d_{j}\right)(h+\log s+3 n(n+1) d) .
\end{aligned}
$$


We can also estimate these parameters through the arithmetic Bernstein-Kushnirenko inequality:

Lemma 4.9 Let $f_{1}, \ldots, f_{s} \in K\left[x_{1}, \ldots, x_{n}\right]$ be polynomials without common zeros in $\mathbb{A}^{n}$. Set $d:=\max _{i} \operatorname{deg} f_{i}$ and $h:=h\left(f_{1}, \ldots, f_{s}\right)$. Also let $\mathcal{V}$ denote the volume of $1, x_{1}, \ldots, x_{n}, f_{1}, \ldots, f_{s}$. Then

- $\delta\left(f_{1}, \ldots, f_{s}\right) \leq \mathcal{V}$

- $\eta\left(f_{1}, \ldots, f_{s}\right) \leq n \mathcal{V}\left(h+\log s+2^{2 n+4} d\right)$.

Proof.- Let $a:=a_{\min }=\left(a_{i j}\right)_{i j} \in \mathbb{Z}^{s \times s}$ and set $q_{i}:=a_{i 1} f_{1}+\cdots+a_{i s} f_{s}$ for $i=1, \ldots, s$. Then $\operatorname{Supp}\left(q_{i}\right) \subset \operatorname{Supp}\left(f_{1}, \ldots, f_{s}\right)$ and so $\mathcal{V}\left(1, x_{1}, \ldots x_{n}, q_{1}, \ldots, q_{s}\right) \leq \mathcal{V}$.

Applying Proposition 2.11 we obtain $\delta \leq \mathcal{V}$ and

$$
\begin{aligned}
\eta & \leq\left(n \max _{i} h\left(q_{i}\right)+2^{2 n+3} \log (n+1) d\right) \mathcal{V} \\
& \leq\left(n(h+\log s+2(n+1) \log (d+1))+2^{2 n+3} \log (n+1) d\right) \mathcal{V} \\
& \leq n \mathcal{V}\left(h+\log s+2^{2 n+4} d\right) .
\end{aligned}
$$

\subsubsection{Proof of Theorem 2}

Modulo the preparation of the input data, the proof of Theorem 2 follows the lines of the example introduced at the beginning of Chapter 4 .

Theorem 4.10 (Intrinsic arithmetic Nullstellensatz)

Let $K$ be a number field and let $f_{1}, \ldots, f_{s} \in \mathcal{O}_{K}\left[x_{1}, \ldots, x_{n}\right]$ be polynomials without common zeros in $I \mathrm{~A}^{n}$. Set $d:=\max _{i} \operatorname{deg} f_{i}$ and $h:=h\left(f_{1}, \ldots, f_{s}\right)$. Also let $\delta$ and $\eta$ denote the degree and the height of the polynomial system $f_{1}, \ldots, f_{s}$.

Then there exist $a \in \mathbb{Z} \backslash\{0\}$ and $g_{1}, \ldots, g_{s} \in \mathcal{O}_{K}\left[x_{1}, \ldots, x_{n}\right]$ such that

- $a=g_{1} f_{1}+\cdots+g_{s} f_{s}$,

- $\operatorname{deg} g_{i} \leq 2 n^{2} d \delta$,

- $h\left(a, g_{1}, \ldots, g_{s}\right) \leq(n+1)^{2}[K: \mathbb{Q}] d\left(2 \eta+(h+\log s) \delta+21(n+1)^{2} d \log (d+1) \delta\right)$.

Proof.- Let $a_{\min }=\left(a_{i j}\right)_{i j} \in \mathbb{Z}^{s \times s}$ be a coefficient matrix which realizes the degree and height of $f_{1}, \ldots, f_{s}$. We have then $\delta=\delta\left(a_{\min }\right), \eta=\eta\left(a_{\min }\right)$ and $h\left(a_{\text {min }}\right) \leq 2(n+1) \log (d+1)$. We set

$$
q_{i}:=a_{i 1} f_{1}+\cdots+a_{i s} f_{s}
$$

for $i=1, \ldots, s$. Then $\left(q_{1}, \ldots, q_{i}\right)$ is a radical ideal of dimension $n-i$ for $i=1, \ldots, t-1$ and $1 \in\left(q_{1}, \ldots, q_{t}\right)$ for some $t \leq \min \{n+1, s\}$.

Also let $b_{k l} \in \mathbb{Z}$ be integers with $h\left(b_{k l}\right) \leq 2(n+1) \log (d+1)$ such that $V_{i}:=V\left(q_{1}, \ldots, q_{i}\right)$ satisfies Assumption 1.5 with respect to the variables

$$
y_{k}:=b_{k 0}+b_{k 1} x_{1}+\cdots+b_{k n} x_{n}
$$

for $i=1, \ldots, t-1$. Set $b:=\left(b_{k 0}\right)_{k} \in \mathbb{Z}^{n}$ and $B:=\left(b_{k l}\right)_{k, l \geq 1} \in \mathrm{GL}_{n}(\mathbb{Q})$, and set $\varphi: \mathbb{A}^{n} \rightarrow \mathbb{A}^{n}$ for the affine map $\varphi(x):=B x+b$. For $j=1, \ldots, t$ we then set

$$
F_{j}(y):=q_{j}(x)=q_{j}\left(\varphi^{-1}(y)\right) \in K\left[y_{1}, \ldots, y_{n}\right]
$$


Thus $F_{1}, \ldots, F_{t}$ are in the hypothesis of Lemma 3.3 with respect to $y_{1}, \ldots, y_{n}$ and we let $P_{1}, \ldots, P_{t} \in$ $K\left[x_{1}, \ldots, x_{n}\right]$ be the polynomials satisfying Bészout identity we obtain there.

Finally, for $i=1, \ldots, s$, we set

$$
p_{i}:=\sum_{j=1}^{t} a_{i j} P_{j}(\varphi(x)) \in K\left[x_{1}, \ldots, x_{n}\right] .
$$

We have $1=p_{1} f_{1}+\cdots+p_{s} f_{s}$.

Now we analyze the degree and the height of these polynomials. We will assume $n, d \geq 2$ as the remaining cases have already been considered in Lemmas 3.7 and 3.8.

Set $W_{l}:=V\left(F_{1}, \ldots, F_{l}\right) \subset \mathbb{A}^{n}$ for $l=1, \ldots, t-1$. We have $W_{l}=\varphi\left(V_{l}\right)$ and so $\operatorname{deg} W_{l}=\operatorname{deg} V_{l}$.

We have also $\operatorname{deg} F_{j}=\operatorname{deg} q_{j} \leq d$ and so

$$
\operatorname{deg} p_{i} \leq \max _{j} \operatorname{deg} P_{j} \leq 2 n d\left(1+\sum_{l=1}^{\min \{n, s\}-1} \operatorname{deg} W_{l}\right) \leq 2 n^{2} d \delta
$$

as $\operatorname{deg} W_{l} \leq \delta$ for $l \leq n-1$.

Now let $v \in M_{K}^{\infty}$ and set $h_{v}:=\max _{i} h_{v}\left(f_{i}\right)$. We have

$$
\begin{aligned}
h_{\infty}\left(\varphi^{-1}\right) & \leq n\left(h_{\infty}(\varphi)+\log n\right)-\log |\operatorname{det} B|_{\infty} \\
& \leq n(2(n+1) \log (d+1)+\log n)-\log |\operatorname{det} B|_{\infty} \\
& \leq 3 n(n+1) \log (d+1)-\log |\operatorname{det} B|_{\infty}
\end{aligned}
$$

Then

$$
\begin{aligned}
h_{v}\left(F_{i}\right) & \leq h_{v}\left(q_{i}\right)+\left(h_{\infty}\left(\varphi^{-1}\right)+2 \log (n+1)\right) \operatorname{deg} q_{i} \\
& \leq h_{v}+2(n+1) \log (d+1)+\log s+\left(3 n(n+1) \log (d+1)-\log |\operatorname{det} B|_{\infty}+2 \log (n+1)\right) d \\
& \leq h_{v}+\log s+(n+1+3 n(n+1)+2 n) d \log (d+1)-\log |\operatorname{det} B|_{\infty} d \\
& \leq h_{v}+\log s+3(n+1)^{2} d \log (d+1)-\log |\operatorname{det} B|_{\infty} d .
\end{aligned}
$$

by Lemma 1.2 (c) and the facts that $\log (n+1) \leq n$ and $\log (d+1) \geq 1$ for $d \geq 2$. Next, applying Lemma 2.6, we obtain

$$
\begin{aligned}
h\left(W_{l}\right) & \leq h\left(V_{l}\right)+(n-l+1)(h(\varphi)+5 \log (n+1)) \operatorname{deg} V_{l} \\
& \leq h\left(V_{l}\right)+n(2(n+1) \log (d+1)+5 \log (n+1)) \operatorname{deg} V_{l} \\
& \leq \eta+n(7 n+2) d \log (d+1) \delta
\end{aligned}
$$

as $\operatorname{deg} W_{l}=\operatorname{deg} V_{l} \leq d \delta$ and $h\left(V_{l}\right) \leq \eta$ for $l=1, \ldots, t-1$. By Lemma 3.3 there exists $\xi \in K^{*}$ such that

$$
\begin{aligned}
h_{v}\left(P_{j}\right) \leq & 2 n d \sum_{l=1}^{t-1} h_{v}\left(W_{l}\right)+\left((n+1) \max _{l} h_{v}\left(F_{l}\right)+2 n(2 n+5) \log (n+1) d\right)\left(1+\sum_{l=1}^{t-1} \operatorname{deg} W_{l}\right) \\
& -\log |\xi|_{v} \\
\leq & 2 n d \sum_{l=1}^{t-1} h_{v}\left(W_{l}\right)+(n+1)^{2}\left(h_{v}+\log s\right) d \delta \\
& +\left(3(n+1)^{4}+2 n^{2}(2 n+5)(n+1)\right) d^{2} \log (d+1) \delta-\log |\mu|_{v}
\end{aligned}
$$


with $\mu:=(\operatorname{det} B)^{(n+1)^{2} d^{2} \delta} \xi \in K^{*}$. Then

$$
\begin{aligned}
h_{v}\left(p_{i}\right) \leq & \max _{j} h_{v}\left(P_{j}\right)+\left(h_{\infty}(\varphi)+2 \log (n+1)\right) \max _{j} \operatorname{deg} P_{j}+2(n+1) \log (d+1)+\log t \\
\leq & 2 n d \sum_{l} h_{v}\left(W_{l}\right)+(n+1)^{2}\left(h_{v}+\log s\right) d \delta \\
& +\left(3(n+1)^{4}+2 n^{2}(2 n+5)(n+1)\right) d^{2} \log (d+1) \delta-\log |\mu|_{v} \\
& +(2(n+1) \log (d+1)+2 \log (n+1)) 2 n^{2} d \delta+2(n+1) \log (d+1)+\log (n+1) \\
\leq & 2 n d \sum_{l} h_{v}\left(W_{l}\right)+(n+1)^{2}\left(h_{v}+\log s\right) d \delta+7(n+1)^{3}(n+2) d^{2} \log (d+1) \delta-\log |\mu|_{v} .
\end{aligned}
$$

Analogously $h_{v}\left(p_{i}\right) \leq 2 n d \sum_{l} h_{v}\left(W_{l}\right)+(n+1)^{2} h_{v} d \delta-\log |\mu|_{v}$ for $v \notin M_{K}^{\infty}$.

Hence

$$
\begin{aligned}
h\left(p_{1}, \ldots, p_{s}\right) \leq & 2 n d \sum_{l} h\left(W_{l}\right)+(n+1)^{2}(h+\log s) d \delta+7(n+1)^{3}(n+2) d^{2} \log (d+1) \delta \\
\leq & 2 n^{2} d \eta+2 n^{3}(7 n+2) d^{2} \log (d+1) \delta+(n+1)^{2}\left(h_{v}+\log s\right) d \delta \\
& +7(n+1)^{3}(n+2) d^{2} \log (d+1) \delta \\
\leq & 2 n^{2} d \eta+(n+1)^{2}\left(h_{v}+\log s\right) d \delta+21(n+1)^{4} d^{2} \log (d+1) \delta .
\end{aligned}
$$

Finally we apply Lemma 1.3 to obtain $a \in \mathbb{Z} \backslash\{0\}$ such that $g_{i}:=a p_{i} \in \mathcal{O}_{K}\left[x_{1}, \ldots, x_{n}\right]$. Thus

$$
a=g_{1} f_{1}+\cdots+g_{s} f_{s}
$$

and the corresponding height estimates are multiplied by $[K: \mathbb{Q}]$.

We derive from this result and the Bézout inequality 4.8 the following estimate in terms of the degree and the height of the input polynomials:

Corollary 4.11 Let $f_{1}, \ldots, f_{s} \in \mathcal{O}_{K}\left[x_{1}, \ldots, x_{n}\right]$ be polynomials without common zeros in $\mathbb{I}^{n}$. Set $d_{i}:=\operatorname{deg} f_{i}$ and assume that $d_{1} \geq \cdots \geq d_{s}$ holds. Also set $d:=d_{1}=\max _{i} \operatorname{deg} f_{i}, h:=$ $h\left(f_{1}, \ldots, f_{s}\right)$, and $n_{0}:=\min \{n, s\}$.

Then there exist $a \in \mathbb{Z} \backslash\{0\}$ and $g_{1}, \ldots, g_{s} \in \mathcal{O}_{K}\left[x_{1}, \ldots, x_{n}\right]$ such that

- $a=g_{1} f_{1}+\cdots+g_{s} f_{s}$,

- $\operatorname{deg} g_{i} \leq 2 n^{2} d \prod_{j=1}^{n_{0}-1} d_{j}$,

- $h\left(a, g_{1}, \ldots, g_{s}\right) \leq 2(n+1)^{3}[K: \mathbb{Q}] d \prod_{j=1}^{n_{0}-1} d_{j}(h+\log s+3 n(n+7) d \log (d+1))$.

\subsubsection{Estimates for the sparse case}

Bernstein-Kushnirenko inequality 4.9 shows that both the degree and the height of a system are controlled by its volume. We derive then from Theorem 4.10 an arithmetic Nullstellensatz for sparse polynomial systems.

Corollary 4.12 (Sparse arithmetic Nullstellensatz)

Let $f_{1}, \ldots, f_{s} \in \mathcal{O}_{K}\left[x_{1}, \ldots, x_{n}\right]$ be polynomials without common zeros in $\mathbb{I}^{n}$. Set $d:=\max _{i} \operatorname{deg} f_{i}$ and $h:=h\left(f_{1}, \ldots, f_{s}\right)$. Also let $\mathcal{V}$ denote the volume of the polynomial system $1, x_{1}, \ldots, x_{n}, f_{1}, \ldots, f_{s}$. Then there exist $a \in \mathbb{Z} \backslash\{0\}$ and $g_{1}, \ldots, g_{s} \in \mathcal{O}_{K}\left[x_{1}, \ldots, x_{n}\right]$ such that 
- $a=g_{1} f_{1}+\cdots+g_{s} f_{s}$

- $\operatorname{deg} g_{i} \leq 2 n^{2} d \mathcal{V}$,

- $h\left(a, g_{1}, \ldots, g_{s}\right) \leq 2(n+1)^{3}[K: \mathbb{Q}] d \mathcal{V}\left(h+\log s+2^{2 n+4} d \log (d+1)\right)$.

Example 4.13 For $1 \leq i \leq s$ we let

$$
f_{i}:=a_{i 0}+a_{i 1} x_{1}+\cdots+a_{i n} x_{n}+b_{i 1} x_{1} \cdots x_{n}+\cdots+b_{i d}\left(x_{1} \cdots x_{n}\right)^{d} \in \mathbb{Z}\left[x_{1}, \ldots, x_{n}\right]
$$

be polynomials of degree bounded by $n d$ without common zeros in $\mathbb{I}^{n}$. Set $h:=\max _{i} h\left(f_{i}\right)$. Also set $\mathcal{P}_{d}:=\operatorname{Conv}\left(0, e_{1}, \ldots, e_{n}, d\left(e_{1}+\cdots+e_{n}\right)\right) \subset \mathbb{R}^{n}$, so that $\mathcal{P}_{d}$ contains the Newton polytope of the polynomials $1, x_{1}, \ldots, x_{n}, f_{1}, \ldots, f_{s}$. Then

$$
\mathcal{V} \leq \operatorname{Vol}\left(\mathcal{P}_{d}\right)=n ! d /(n-1) !=n d .
$$

We conclude that there exist $a \in \mathbb{Z} \backslash\{0\}$ and $g_{1}, \ldots, g_{s} \in \mathbb{Z}\left[x_{1}, \ldots, x_{n}\right]$ such that

- $a=g_{1} f_{1}+\cdots+g_{s} f_{s}$,

- $\operatorname{deg} g_{i} \leq 2 n^{4} d^{2}$,

- $h(a), h\left(g_{i}\right) \leq 2 n^{2}(n+1)^{3} d^{2}\left(h+\log s+n 2^{2 n+4} d \log (n d+1)\right)$.

This estimate is sharper than the one given by Theorem 1.

\section{References}

[1] C. A. Berenstein, D. C. Struppa, Recent improvements in the complexity of the effective Nullstellensatz, Linear Algebra Appl. 157 (1991), pp. 203-215.

[2] C. A. Berenstein, A. YGer, Effective Bézout identities in $\mathbb{Q}\left[x_{1}, \ldots, x_{n}\right]$, Acta Math. 166 (1991), pp. $69-120$.

[3] C. A. Berenstein, A. YGer, Residue calculus and effective Nullstellensatz, Amer. J. Math. 121 (1999), pp. 723-796.

[4] D. N. Bernstein, The number of roots of a system of equations, Functional Anal. Appl. 9 (1975), pp. $183-185$.

[5] J.-B. Bost, H. Gillet, C. Soulé, Height of projective varieties and positive Green forms, J. Amer. Math. Soc. 7 (1994), pp. 903-1027.

[6] W. D. Brownawell, Bounds for the degrees in the Nullstellensatz, Ann. of Math. 126 (1987), pp. $577-591$.

[7] L. Caniglia, A. Galligo, J. Heintz, Borne simplemente exponentielle pour les degrés dans le théorème des zéros sur un corps de charactéristique quelconte, C. R. Acad. Sci. Paris 307 (1988), pp. $255-258$.

[8] J. Canny, I. Emiris, A subdivision-based algorithm for the sparse resultant, to appear in J. Symbolic Comput.

[9] L. Ein, R. Lazarsfeld, A geometric effective Nullstellensatz, Invent. Math. 137 (1999), pp. $427-448$.

[10] G. Faltings, Diophantine approximation on abelian varieties, Ann. of Math. 133 (1991), pp. 549-576. 
[11] N. Fitchas, A. Galligo, Nullstellensatz effectif et conjecture de Serre (théorème de Quillen-Suslin) pour le Calcul Formel, Math. Nachr. 149 (1990), pp. 231-253.

[12] N. Fitchas, M. Giusti, F. Smietanski, Sur la complexité du théorème des zéros, in J. Gudat et. al., eds., Approximation and optimization 8, Peter Lange Verlag, 1995, pp. 247-329.

[13] W. Fulton, Intersection theory, Erg. Math., 3. Folge., 2. Bd., Springer-Verlag, 1984.

[14] I. M. Gelfand, M. M. Kapranov, A. V. Zelevinsky, Discriminants, resultants, and multidimensional determinants, Birkhäuser, 1994.

[15] M. Giusti, K. Hägele, J. Heintz, J. L. Montaña, L. M. Pardo, J. E. Morais, Lower bounds for diophantine approximation, J. Pure Appl. Algebra 117 \& 118 (1997), pp. 277-317.

[16] M. Giusti, J. Heintz, J. E. Morais, J. Morgenstern, L. M. Pardo, Straight-line programs in geometric elimination theory, J. Pure Appl. Algebra 124 (1998), pp. 101-146.

[17] M. Giusti, J. Heintz, J. Sabia, On the efficiency of effective Nullstellensätze, Comput. Complexity 3 (1993), pp. 56-95.

[18] K. Ḧ̈GELE, Intrinsic height estimates for the Nullstellensatz, Ph.D. Thesis, Univ. Cantabria, 1998.

[19] K. HäGele, J. E. Morais, L. M. Pardo, M. Sombra, On the intrinsic complexity of the arithmetic Nullstellensatz, to appear in J. Pure Appl. Algebra.

[20] J. Heintz, Definability and fast quantifier elimination in algebraically closed fields, Theoret. Comput. Sci. 24 (1983), pp. 239-277.

[21] J. Heintz, C.-P. SchnorR, Testing polynomials which are easy to compute, Monografie Enseignement Math. 30 (1982), pp. 237-254.

[22] G. Hermann, Der Frage der endlich vielen Schritte in der Theorie der Polynomideale, Math. Ann. 95 (1926), pp. 736-788.

[23] S. Ji, J. Kollár, B. Shiffman, A global Lojasiewicz inequality for algebraic varieties, Trans. Amer. Math. Soc. 329 (1992), pp. 813-818.

[24] M. M. Kapranov, B. Sturmfels, A. V. Zelevinsky, Chow polytopes and generalized resultants, Duke Math. J. 67 (1992), 189-218.

[25] P. KoIRAn, Hilbert's Nullstellensatz is in the polynomial hierarchy, J. Complexity 12 (1996), pp. 273-286.

[26] J. Kollár, Sharp effective Nullstellensatz, J. Amer. Math. Soc. 1 (1988), pp. 963-975.

[27] J. KollÁR, Effective Nullstellensatz for arbitrary ideals, Manuscript, Univ. Utah, 1998. E-prints: math. AG/9805091.

[28] T. Krick, L. M. PARdo, Une approche informatique pour l'approximation diophantienne, C. R. Acad. Sci. Paris 318 (1994), pp. 407-412.

[29] T. Krick, L. M. PARdo, A computational method for diophantine approximation, Progress in Math. 143, Birkhäuser, 1996, pp. 193-253.

[30] T. KRICK, J. SABIA, P. Solernó, On intrinsic bounds in the Nullstellensatz, AAECC J. 8 (1997), pp. 125-134.

[31] E. Kunz, Kähler differentials, Adv. Lect. in Math., Vieweg-Verlag, 1986.

[32] A. G. Kushnirenko, Newton polyhedra and Bézout's theorem, Functional Anal. Appl. 10 (1976), pp. 233-235. 
[33] S. LANG, Algebraic number theory, Addison-Wesley, 1970.

[34] D. LazARD, Algèbre linéaire sur $k\left[x_{1}, \ldots, x_{n}\right]$ et élimination, Bull. Soc. Math. France 105 (1977), pp. 165-190.

[35] D. H. Lehmer, Factorization of certain cyclotomic functions, Ann. of Math. 34 (1933), pp. 461-479.

[36] P. Lelong, Mesure de Mahler et calcul des constantes universelles pour les polynômes de $n$ variables, Math. Ann. 299 (1994), pp. 673-695.

[37] P. Lelong, L. Gruman, Entire functions of several complex variables, Grundl. Math. Wiss. 282, Springer-Verlag, 1986.

[38] K. MahleR, On some inequalities for polynomials in several variables, J. London Math. Soc. 37 (1962), pp. 341-344.

[39] V. Maillot, Géométrie d'Arakelov des variétés toriques et fibrés en droites intégrables, to appear in Mém. Soc. Math. France.

[40] D. W. Masser, G. Wüstholz, Fields of large trascendence degree generated by values of elliptic functions, Invent. Math. 72 (1983), pp. 407-464.

[41] H. Matsumura, Commutative ring theory, Cambridge Univ. Press, 1986.

[42] L. M. PARdo, How upper and lower bounds meet in elimination theory, in G. Cohen, M. Giusti and T. Mora, eds., Proc. AAECC-11, Lect. Notes. Comput. Sci. 948, Springer-Verlag, 1995, pp. 33-69.

[43] P. Pedersen, B. Sturmfels, Product formulas for resultants and Chow forms, Math. Z. 214 (1993), pp. 377-396.

[44] P. Philippon, Critères pour l'indépendance algébrique, Publ. Math. IHES 64 (1986), pp. 5-52.

[45] P. Philippon, Dénominateurs dans le théorème des zeros de Hilbert, Acta Arith. 58 (1990), pp. 1-25.

[46] P. Philippon, Sur des hauteurs alternatives, I, Math. Ann. 289 (1991), pp. 255-283; II, Ann. Inst. Fourier 44 (1994), pp. 1043-1065; III, J. Math. Pures Appl. 74 (1995), pp. 345-365.

[47] J. SABiA, P. Solernó, Bounds for traces in complete intersections and degrees in the Nullstellensatz, AAECC J. 6 (1995), pp. 353-376.

[48] P. Solernó, Effective Lojasiewicz inequalities in semialgebraic geometry, AAECC 2 (1989), pp. 1-14.

[49] M. SombrA, Bounds for the Hilbert function of polynomial ideals and for the degrees in the Nullstellensatz, J. Pure Appl. Algebra 117 \& 118 (1997), pp. 565-599.

[50] M. Sombra, A sparse effective Nullstellensatz, Adv. Appl. Math. 22 (1999), pp. 271-295.

[51] M. SombrA, Estimaciones para el teorema de ceros de Hilbert, Ph.D. Thesis, Univ. Buenos Aires, 1998.

[52] C. Soulé, Géometrie d'Arakelov et nombres trascendants, J. Arithmétiques, Luminy'89, Asterisque 198-200 (1991), pp. 355-371.

[53] B. Sturmfels, Sparse elimination theory, in D. Eisenbud and L. Robbiano, eds., Computational algebraic geometry and commutative algebra, Cambridge Univ. Press, 1993, pp. 377-396.

[54] B. Sturmfels, Gröbner bases and convex polytopes, Univ. Lect. Series 8, Amer. Math. Soc., 1996.

[55] B. Teissier, Résultats récents d'algèbre commutative effective, Sém. Bourbaki 718, Astérisque 189 190, pp. 107-131, Soc. Math. France, 1991. 
Teresa Krick: Departamento de Matemática, Universidad de Buenos Aires, Ciudad Universitaria, 1428 Buenos Aires, Argentina

E-mail : krick@dm.uba.ar

Luis Miguel Pardo: Departamento de Matemáticas, Estadística y Computación, Universidad de Cantabria, E-39071 Santander, España

E-mail: pardo@matesco.unican.es

Martín Sombra: Departamento de Matemática, Universidad Nacional de La Plata, Calle 50 y 115, 1900 La Plata, Argentina, and School of Mathematics, Institute for Advanced Study, Princeton NJ 08540, USA,

E-mail: sombra@mate.unlp.edu.ar, sombra@ias.edu 